\title{
German Congress of Laboratory Medicine: 15th Annual Congress of the DGKL (German Society of Clinical Chemistry and Laboratory Medicine) and the 3rd Symposium on Biomedical Analysis
}

\author{
Mannheim, Germany, September 26-29, 2018 \\ Under the auspices of \\ International Federation of Clinical Chemistry and Laboratory Medicine \\ European Federation of Clinical Chemistry and Laboratory Medicine \\ Congress Presidents \\ Eberhard Wieland (Stuttgart, Germany) \\ Matthias Orth (Stuttgart, Germany) \\ Hansjörg Baum (Ludwigsburg, Germany) \\ Christiane Maschek (DVTA, Hamburg, Germany) \\ Sponsor of Abstract awards \\ Dr. Neumann \& Kindler Ltd. \& Co. KG (Bochum, Germany)
}

\begin{abstract}
Scientific Committee
Ahmad-Nejad Parviz; Aufenanger, Johannes; Bauer Matthias; Baum Hannsjörg; Bertsch Thomas; Birschmann Ingvild; Bobrowski Andreas; Brand Korbinian; Bühling Frank; Burkhardt Ralph; Ceglarek Uta; Chavakis Triantafyllos; Danckwardt Sven; Fischer Andreas; Fraunberger Peter; Gässler Norbert; Gerritzen Andreas; Griesmacher Andrea; Gurr Eberhard; Häcker Georg; Haferlach Thorsten; Hallbach Jürgen; Haselmann Verena; Haushofer Alexander; Heeg Klaus; Hersberger Martin; Hoffmann Georg; Hofmann Walter; Holdenrieder Stefan; Hunfeld Klaus-Peter; Isermann Berend; Junker Ralf; Kaap-Fröhlich Sylvia; Kachler Marco; Kessler Harald; Kiehntopf Michael; Klouche Mariam; Knabbe Cornelius; Kohse Klaus P.; Kratzsch Jürgen; Lackner Karl; Lichtinghagen Ralf; Luppa Peter; Magnussen Karin; Maschek Christiane; Miethke Thomas; Nauck Matthias; Neumaier Michael; Nofer Jerzy-Roch; Orth Matthias; Peetz Dirk; Peter Andreas; Petersmann Astrid; Rauh Manfred; Renné Thomas; Renz Harald; Ruland Jürgen; Schimanski Sven; Schuff-Werner Peter; Siegert Gabriele; Stoffel-Wagner Birgit; Streichert Thomas; Tauber Rudolf; Teupser Daniel; Uhr Manfred; Vogeser Michael; von Ahsen Nicolas; von Eckardstein Arnold; Walter Ulrich; Walter Philipp; Wiegel Bernhard; Wieland Eberhard
\end{abstract}

*)These abstracts have been reproduced directly from the material supplied by the authors, without editorial alteration by the staff of this Journal. Insufficiencies of preparation, grammar, spelling, style, syntax, and usage are the authors. 


\title{
Talks
}

\section{Infektiologie \& Mikrobiologie: Aktuelle Aspekte}

\author{
V01 - Talk Hunfeld \\ Labordiagnostik der Lyme-Borreliose: Aktueller Stand
}

Klaus-Peter Hunfeld

Krankenhaus Nordwest, Labormedizin, Mikrobiologie und Krankenhaushygiene, Frankfurt am Main, Germany

This presentation is directed at physicians and laboratory personnel in private practice and clinics who treat and diagnose Lyme borreliosis (LB) in patients as part of their daily work. A major objective of the talk is to bring together background information on Borrelia (B.) burgdorferi sensu lato (s.l.) and basic clinical knowledge of LB, which is one of the most frequently reported vector-borne diseases in the Northern hemisphere. The goal is to provide practical guidance for clinicians and for laboratory physicians, and scientists for a better understanding of current achievements and ongoing obstacles in the laboratory diagnosis of LB, an infectious disease that still remains one of the diagnostic chameleons of modern clinical medicine. Moreover, in bringing together current scientific information from guidelines, reviews, and original papers, this presentation will provide recommendations for selecting the appropriate tests in relation to the patient's stage of disease to achieve effective, stage-related application of current direct and indirect laboratory methods for the detection of B. burgdorferi s.l.. Additionally, the talk aims to discuss the current state of the art concerning the diagnostic potential and limitations of the assays and test methods currently in use to optimize LB patient management and provide insight into the possible future prospects of this rapidly changing area of laboratory medicine.

\section{V02 - Talk Ziesing}

\section{Standardisierte Empfindlichkeitstestung im Licht der unterschiedlichen Regelwerke: Wohin geht die Reise?}

Stefan Ziesing

Medizinische Hochschule Hannover, Institut für medizinische Mikrobiologie und Krankenhaushygiene, Hannover, Germany

Microbiology laboratory results from antibiotic susceptibility testing (AST) are of major importance for clinical practice as they determine the choice of the most appropriate antibiotic regime. The impact is enhanced due to the ever growing incidence of multidrug resistant bacteria. Several AST techniques are available such as measurement of the diameters of inhibition zones in disk diffusion or determination of minimal inhibitory concentrations (MIC), most often in micro dilution assays and some other techniques. These results are interpreted for the in vivo setting as "resistant”, "intermediate susceptible" or "susceptible" using given breakpoints. These categories allow selecting suitable antibiotics for antimicrobial chemotherapy. Breakpoints for interpretation are substance-specific, have to consider approved dosages, pharmacokinetic and -dynamic properties, ability to discriminate between wildtype strains and those with resistance mechanisms, MIC distributions for different genera and species of bacteria and not least correlations between in vitro findings and dose dependent overall clinical response. As every AST is a measurement in a complex biological system a highly defined standard must be followed to gain precise and reproducible results. Currently the eldest standard used in German laboratories is the US American (CLSI) standard. Since 2010, a European standard (EUCAST) is widely accepted and has been established not only in European countries. However, despite the harmonization attained by EUCAST still some national issues remain, i.e. different approved dosages. Therefore national EUCAST committees have been established in recent years. The presentation will demonstrate the current status of both, CLSI and EUCAST, current usage, development of these standards and selected samples illustrating the interaction between AST users and standardization committees. Finally, implications for external quality assessment in a world with different AST standards will be shown.

\section{Labordiagnostik bei Herz-Kreislauferkrankungen}

\section{V03 - Talk Li}

\section{Biomarker des oxidativen Stress}

Huige Li

Universitätsmedizin Mainz, Institut für Pharmakologie, Mainz, Germany

Oxidative stress plays an important role in the pathogenesis of various diseases including cardiovascular disease, metabolic disease, neurodegenerative disease, and cancer. However, quantification of oxidative stress in the clinical setting is challenging. The half-life of reactive 
oxygen species is very short. Human tissues and cells, in which oxidative stress occurs, are not readily available for quantification. A feasible approach is to measure biomarkers of oxidative stress in blood or urine samples. These include molecules that are modified by reactive oxygen species and molecules of the antioxidant system that change in response to increased redox stress. Examples are oxidative modification products of proteins (nitrotyrosine, S-glutathionylation, oxidized low-density lipoprotein), lipids (F2-isoprostanes and malondialdehyde), and DNA/RNA (7,8-dihydro-8-oxo-2'-deoxyguanosine and 7,8-dihydro-8-oxoguanosine). The transcription factor NRF2 and glutathione peroxidase-1, on the other hand, are markers of antioxidant defense. The advantages and disadvantages of such molecules as potential biomarkers of oxidative stress are summarized and discussed.

\title{
S2 Seminar
}

\section{V04 - Talk Kaap-Fröhlich}

\section{Wissenschaftliches und evidenzbasiertes Arbeiten im medizinischen Labor - Einführung in die Literaturrecherche}

\author{
Sylvia Kaap-Fröhlich
}

Universität Zürich, Zürich, Switzerland

\begin{abstract}
Ausgehend vom Grundverständnis wissenschaftlichen und evidenzbasierten Arbeiten werden im Workshop die Anforderungen an eine selektive und eine systematische Literaturrecherche definiert.

Die Möglichkeiten zum Wiederfinden und zum Suchen von Literatur werden anhand der medizinischen Literaturdatenbank Medline (via Pumed-Oberfläche) demonstriert. Dabei wird u.a. auf verschiedene Filter, MeSH-Terms, Tags und Boolsche Operatoren sowie auf die Besonderheiten zum Auffinden von laborrelevanter Literatur eingegangen. An Beispielfragen werden verschiedene Suchstrategien geübt. Die Teilnehmenden können anhand eigener Fragestellungen Literatur suchen und die Suchstrategien mit anderen Workshopteilnehmenden diskutieren. Eine Einführung zur Beschaffung der Literatur rundet den Workshop ab.
\end{abstract}

\section{Mythen und Fakten in der pädiatrischen Labormedizin}

\section{V05 - Talk Rauh}

\section{Abschätzung der GFR bei Kindern}

\author{
Manfred Rauh \\ Universitäts Erlangen, Kinder- und Jugendklinik, Klinisches und Infektionsserologisches Labor, Erlangen, Germany
}

The glomerular filtration rate (GFR) is the best indicator of renal function in children and adolescents, and its estimation has a long history. Having an accurate means of determining GFR is critical for diagnosing acute and chronic kidney impairment and monitoring for side effects of medications. As evidence is increasing that cystatin C-based formulae can be an alternative to creatinine-based measurements, suggested GFR estimating equations are growing in numbers. The current limitations and difficulties of GFR estimation including potential dosing errors are discussed. Cystatin C-based equations can be used as alternatives to creatinine-based estimations when the appropriate laboratory method has been applied. The aim is to create awareness of the selection of a GFR estimation formula that is most appropriate for the lab test method used and the respective patient collective.

\section{V06 - Talk Baumann}

\section{Die Herausforderung Neonatale Sepsis}

Philipp Baumann

Universitäts-Kinderspital Zürich, Intensivmedizin \& Neonatologie, Zürich, Germany

Neonatal sepsis is a dangerous clinical syndrome consisting of systemic signs of infection and isolation of a bacterial pathogen from the bloodstream. Two to $4 \%$ of affected children die and mortality depends on both gestational age with preterm infants being the more vulnerable ones (mortality up to $20 \%$ ) and specific pathogens (mortality with E. coli 6 - $10 \%$, with Group B Streptococcus $1-2 \%$ ). The condition is divided into early-onset ( $<72 \mathrm{~h}$ after birth) and late-onset sepsis ( $72 \mathrm{~h}-28 \mathrm{~d}$ after birth). Early-onset sepsis is usually caused by vertical transmission via ascending contaminated amniotic fluid or during vaginal birth from pathogens in the mother's genital tract. However, lateonset infections also occur after vertical transmission with initial asymptomatic neonatal colonization and delayed systemic infection or due to horizontal transmission from contact with environmental sources. 
As neonatal sepsis may progress very rapidly and early antibiotic treatment is life-saving, physicians often start empiric treatment at disease onset and cancel the treatment within $36-48 \mathrm{~h}$, when the course of the child develops favourably. A quick and reliable laboratory parameter would be crucial to avoid antibiotic overtreatment in this situation, but until now, no such marker is available. Currently, the only laboratory result establishing the diagnosis of neonatal sepsis is a positive blood culture. Unfortunately, time to positivity is $24-36 \mathrm{~h}$ and blood culture results may be blurred by contamination with skin flora. If the difficult diagnosis of early-onset sepsis has to be made directly after birth we can only estimate the likelihood of sepsis taking into account risk factors or signs of infection and weak components of the complete blood count, i.e. absolute neutrophil count and ratio of immature to total neutrophil counts. Both parameters are highly variable and more useful for ruling out sepsis than for ruling in. Sequential assessments of CRP may help support a diagnosis of sepsis, but predictive values are poor as a number of non-infectious factors drive CRP after birth (e.g. stressful delivery, perinatal asphyxia). Further, pro- and anti-inflammatory cytokines such as interleukin-4, -6, -10 and tumor necrosis factor-alpha increase in infections, but these react also non-specifically to other stimuli. Recently, procalcitonin evolved as a sepsis marker and was tested for neonatal sepsis as well, but it revealed only moderate sensitivities (72 - $79 \%$ ) and specificities (72 - $90 \%$ ). However, procalcitonin seems to be valuable in individual guidance of antibiotic treatment, as a recent large multicentre study showed a significant reduction of treatment duration under procalcitonin guidance.

Despite the advances in research on diagnosis, prevention and treatment of neonatal sepsis, the paediatrician with a newborn infant in a high-risk situation still has to make his individual treatment decision with the help of suboptimal diagnostic markers.

\title{
Workshop Crashkurs
}

\section{V07}

\section{Crashkurs Biostatistik mit R}

Georg Hoffmann'; Frank Klawonn²; Christof Winter ${ }^{3}$; Andreas Bietenbeck ${ }^{3}$

${ }^{1}$ Trillium GmbH, Grafrath, Germany; ${ }^{2}$ Helmholtzzentrum für Infektionsforschung, Bioinformatik und Statistik, Braunschweig, Germany; ${ }^{3}$ Klinikum rechts der Isar der TU München, Institut für Klinische Chemie und Pathobiochemie, München, Germany

$\mathrm{R}$ is the world language of statistics and bioinformatics. In just three hours, participants will be introduced to this powerful program environment, which can do so much more than Excel or SPSS. The focus of the workshop will be on statistical tests and illustrative graphics for reports, lectures and publications. The content of the course is based on a list of topics proposed by the AG Bioinformatics for advanced training as a clinical chemist [1].

The program will be divided into three parts with many practical exercises:

1) Hoffmann: Introduction to $\mathrm{R}$ and RStudio, descriptive statistics with simulated data (e. g. mean values and medians, quartiles and boxplots, density curves and quantile-quantile plots, pie charts and scatterplots)

2) Klawonn: Inferential statistics with real data (e. g. parametric and nonparametric comparison of two samples, regression and correlation, classification and ROC analysis)

3) Bietenbeck and Winter: Practical exercises in group work with examples and typical questions from the laboratory routine.

Requirements for participation are:

- WLAN-enabled laptop

- Working installation of R (www.r-project.org) and RStudio (www.rstudio.com)

[1] AG Bioinformatik. Weiterbildungsinhalte aus der Biostatistik und Bioinformatik. KCM 2017, 48, 58-60.

\section{Inflammation}

\section{V08 - Talk Scheiermann}

\section{Influence of time-of-day on leukocyte migration and function}

\author{
Christoph Scheiermann \\ LMU München, Walter-Brendel-Zentrum für Experimentelle Medizin, Planegg-Martinsried, Germany
}

Leukocyte infiltration of tissues from the blood is a critical step of the immune system to protect against pathogens and provide tissue repair. Although various molecules have been implicated in these migratory processes, it is unknown how this fundamental process of immunity is being regulated at the organismal level at different times. Here, we describe mechanisms that govern a non-continuous, 
oscillatory behavior in the infiltration of immune cells to different tissues in the mouse. We screened multiple adhesion molecules and chemokine receptors on various leukocyte subpopulations in addition to molecules expressed by endothelial cells in vascular beds of multiple organs. Our data indicate the existence of a leukocyte- and tissue-specific oscillatory molecular signature in the expression of pro-migratory factors. Specifically, we show that lymphocyte trafficking through lymph nodes and lymph occurred in a circadian manner. Lymphocyte homing to lymph nodes peaked at night onset, with cells leaving the tissue during the day. This resulted in strong oscillations in lymphocyte cellularity in lymph nodes and efferent lymphatic fluid. Using lineage-specific genetic ablation of circadian clock function we demonstrated this to be dependent on rhythmic expression of promigratory factors on lymphocytes. Diurnal oscillations in adaptive immune responses were observed after bacterial and viral infections. In addition, disease severity oscillated after immunization to induce experimental autoimmune encephalomyelitis (EAE). These rhythms were abolished by genetic disruption of $\mathrm{T}$ cell clocks, demonstrating a circadian regulation of lymphocyte migration through lymph nodes with time-of-day of immunization being critical for adaptive immune responses weeks later.

Funded by the European Research Council (CIRCODE) and the German Research Foundation (Emmy Noether SCHE 1645/2-1 and SFB914 projects B09 and Z03).

\title{
update gynäkologische Endokrinologie
}

\section{V09 - Talk Ludwig}

\author{
Laborärztliche Befundung in der gynäkologischen Endokrinologie
}

Jochen Ludwig

Laborärzte Sindelfingen, Endokrinologie, Sindelfingen, Germany

The interpretation of laboratory values represents an important interface between the laboratory physician and gynecologists. However interpretation of the findings requires anamnestic data such as the menstrual cycle day or drug therapy. In addition a clinical question must be obvious otherwise interpretation of the findings is not possible. In addition to the lab order, close cooperation and good communication between laboratory doctors and gynecologists are also necessary. The laboratory physician can provide important help with his knowledge and experience regarding individual laboratory methods. The gynecologists also demand close collaboration with the laboratories for a good patient care.

The lecture will show how the laboratory physician can provide the gynecologists with sufficient individual interpretation favourable to a targeted individual interpretation. It is shown which prerequisites are necessary and how the laboratory doctor acquires the knowledge necessary for the interpretation of the findings. Current questions are discussed as well as the elaboration of solutions for specific questions on the basis of case histories.

In addition to specific courses, working groups and expert networks can help to discuss complex gynecological endocrinological issues and provide an individual solution for an optimal interpretation of the findings.

\section{V10 - Talk von Ahsen}

\section{Sinn und Unsinn der Lutealphasendiagnostik}

\author{
Nicolas von Ahsen \\ Universitätsmedizin Göttingen, Abt. Klinische Chemie, Göttingen, Germany
}

It is important in the work-up of infertility to prove ovulation and, by that, exclude anovulatory infertility. The first half of the menstrual cycle leads to an ovulatory follicle with increasing estradiol concentration and endometrial proliferation. The luteal phase, typically 14 days in length, starts after the ovulation. It's hallmark hormone is progesterone which is promoting the endometrial receptivity by secretory transformation. However, interpretation of hormone levels over the cycle is complicated due to variation in cycle length and pulsatility. It is common practise to measure midluteal estradiol and progesterone levels to infer on the "luteal phase quality". A rule-in / rule-out algorithm is recommended for the interpretation. Any measured progesterone concentration $>3 \mu \mathrm{g} / \mathrm{L}$ gives evidence of an ovulatory cycle (rule-in). A single mid-luteal estradiol $>75 \mathrm{ng} / \mathrm{L}$ and progesterone $>8 \mu \mathrm{g} / \mathrm{L}$ rules out luteal phase deficiency. It must be kept in mind that anovulation is not rare during the reproductive years and can occur in up to $1 / 3$ of cycles. It is mandatory to repeat the luteal phase hormone panel in further cycles before a diagnosis of anovulation is made, even more when the cycle length is normal. If repeated testing confirms the luteal phase deficiency further testing for underlying endocrine causes is done. This includes testing for thyroid disorders, ovarian reserve, hyperandrogenemia and hyperprolactinemia. A mid-luteal measurement of estradiol and progesterone is a simple screening test for cycle relevant endocrine disorders. 


\title{
V11 - Talk Kappert
}

\author{
Hormonelle Kontrazeption bei Thrombophilie
}

Günther Kappert

Gerinnungszentrum Rhein-Ruhr, Dres. Rott, Halimeh und Kappert, Duisburg, Germany

In Germany approximally 20 million women are in their reproductive age. One third of them are taking combined oral contraceptives (COC). The basic risk for thromboembolism (TE) in women of this age is low, but rises during pregnancy or when combined hormonal contraceptives (CHC) are used. Therefore, women have a higher risk than men for TE until the age of 35, after that time point the risk remains equal.

Elevation of the thrombotic risk depends on the selected contraceptive. Older COC with norgestimat/levonorgestrel have a lower risk than newer COC. Consequently, these older COC are the first choice in many guidelines. Newer generations of contraceptives should only be prescribed, if a secondary reason other than contraception exists. Furthermore, COC with cyproteronacetat are only indicated for hirsutism and acne vulgaris in Germany, although they have a reliable contraceptive effect. The risk of newer COC containing Estradiol and not Ethinylestradiol is not clear due to missing data. The use of hormonal contraception with gestagen only does not contain any significant increase of the risk for TE with the exception of depot medroxyprogesterone acetate. Emergency contraception which only contains Levonorgestrel or Ulipristalacetat does not result in a higher risk for TE and can be given to women with thrombophila.

So oral desogestrel or levonorgestrel only contraceptives, intrauterine device (IUD) and Etonogestrel sticks are the contraception of choice in women suffering from Thrombophilia.

The screening for Thrombophilia is not indicated in every woman with the wish for contraception. This should be restricted to certain cases, e. g. to women with a positive history for TE or with close relatives suffering from TE in a young age.

\section{Qualität im medizinisch-diagnostischen Laboratorium}

\section{V12 - Talk Schwettmann}

\author{
Überwachung der analytischen Qualität - das norwegische Modell
}

Lutz Schwettmann

Helse Møre og Romsdal, Avdeling for medisinsk biokjemi, Aalesund, Norway

Quality improvement of laboratory investigations is historically of high importance in Scandinavia. In Norway, Noklus (Norwegian Quality Improvement of Laboratory Examinations) has focussed on improvement of the whole laboratory testing process for more than 25 years. Noklus is a non-profit organization which aims to ensure that all laboratory analyses that are ordered, performed and interpreted will safeguard the patient's needs for investigation, treatment and follow-up. Noklus improves quality in medical laboratories in different ways. Funded by the Norwegian Medical Association through a quality improvement fund, Noklus offers access to external quality assessment programs to all Norwegian GP surgeries, hospital laboratories, nursing homes and other health care institutions. Participation is free of charge for the public GPs. For private GPs, nursing homes, occupational physicians and hospital laboratories, participation is charged. The participation in external quality assessment programs is voluntary. Today, $99 \%$ of the Norwegian GP laboratories, $95 \%$ of the nursing homes and all hospital laboratories participate in external quality assessment organized by Noklus. The nationwide surveyed results are used for central supervision of changes in quality, for example between different reagent lots. This enables an efficient action in case of unacceptable changes in quality.

Noklus provides guidance and tuition through site visits, telephone consultations and courses. For this purpose, more than 100 consultants composed of medical technical assistants and specialists in laboratory medicine, are located nationwide. The local presence in all states enables a close communication with the laboratories. Because of a central and nationwide registration of all results of external quality assessment, the Noklus representatives have a good knowledge of the pros and cons of the devices provided on the market and they can assist the laboratories in choosing appropriate devices.

The Scandinavian cooperative SKUP (Scandinavian Evaluation of Laboratory Equipment for Point of Care Testing) was initiated by Noklus and provides objective and manufacturer-independent information about analytical quality and user-friendliness of laboratory equipment. SKUP tests POCT devices by standardized criteria and generates test reports which can aid laboratories in the selection of appropriate instruments in the framework of new acquisition or replacement. Further, the reports can be used by the manufacturers for documentation of quality of their methods and instruments.

After more than 25 years of activity it can be documented that the analytical quality performed by POCT- and automated instruments in- and outside the Norwegian hospitals, was substantially improved. 


\title{
V13 - Talk Thienpont
}

\author{
Percentiler and Flagger - On-line monitoring of test stability and comparability
}

Linda M. Thienpont; Sverre Sandberg'; Dietmar Stöckl

Rennertshofen, Germany; ${ }^{1}$ Haraldsplass Deaconess Hospital, Bergen, Norway; Rennertshofen, Germany

Background: In laboratory medicine, stability of analytical performance and comparability of results across assays/in-vitro diagnostic (IVD) manufacturers are essential for adequate patient management. To monitor these attributes, laboratories perform internal quality control (IQC) and rely on third-party external quality assessment (EQA). However, it is generally recognized that IQC/EQA observations do not necessarily reflect the behavior of an assay with patient samples due to potential commutability issues of the traditionally used sample materials.

Methods: Because of these limitations, Thienpont \& Stöckl Wissenschaftliches Consulting GbR developed 2 new and (up to now) freely available quality management tools called the "Percentiler" and "Flagger". Both make use of results from patients' samples. The concept of the Percentiler is that participants send electronically instrument-specific daily medians preferably from outpatients. Peer grouping by assay/IVD manufacturer is done. The participants have access to a user interface via a specific login/password, where they can see/download per analyte a plot of the course of their instrument-specific moving medians compared to their peer and reflected against stability limits. Where possible, these are guided by biological variation. Also a report comparing peer group medians is regularly provided. The concept of the Flagger is on-line monitoring of the stability of the flagging rate (\% hypo/hyper) against cut-offs used in the individual laboratory. The Flagger stability limits are based on a concept that investigates the effect of a bias (in fractions of the biological variation) on surrogate medical decisions. Several examples of observations from the combined use of both tools will be shown/discussed.

Results: The Percentiler allows the participants to monitor the mid- to long-term stability of their performance; it also shows whether the instruments in a laboratory sufficiently agree with each other and with the peer. The report with peer group medians illustrates how well commercial assays compare and/or are significantly different. The Flagger directly translates the impact of analytical instabilities on the flagging rate. Based on the developers' experience, it seems that for clinical chemistry assays calibration, lot changes, and instability of electrodes are the predominant causes for high analytical variation, while for immunoassays, also within-lot instabilities have an impact; the relevance of biased results on surrogate medical decisions ranges from negligible to pronounced.

Summary: The asset of both Percentiler and Flagger is in the fact that they rely on results from perfectly commutable samples. This makes that the Percentiler is a complementary IQC tool to confirm/disprove observations from IQC materials. The peer group reports are a complementary EQA tool with the potential to build a global evidence base on mid- to long-term comparability (and stability thereof) of assays across manufacturers. The Flagger serves as an additional quality management tool for the analytical phase by bridging in a certain way the "medium-level” hierarchy for setting analytical specifications (biological variation) and the "top-level" (clinical outcomes). Noklus is currently exploring the value of the applications in Scandinavia and outside the borders, as they plan to take over the project latest beginning of 2019. The first 2 authors will continue on the project as consultants.

\section{Neue organisatorische und klinische Anwendungen von POCT-Methoden}

\section{V14 - Talk Oschwald-Häg}

\section{Organisatorische Implementierung von POCT-Methoden in einem Krankenhaus}

Barbara Oschwald-Häg

Ortenau Klinikum Offenburg-Gengenbach, Zentrallaboratorium, Offenburg, Germany

\section{POCT}

standortübergreifende Vernetzung von

Blutgase-und Blutzuckermessgeräten

POCT (point of care testing), zu deutsch Patienten-nahe-Sofortdiagnostik, ist in einer Klinik mit den vielfältigen Fachbereichen, wie Notaufnahmen, Intensivabteilung, so wie auch auf Stationen, nicht mehr wegzudenken.

Der Grund für POCT ist:

Bei lebensbedrohlichen Notfallsituationen so zeitnah wie möglich aus Laborwerten direkt therapeutische Konsequenzen ableiten zu können.

Die Analytischen und diagnostischen Vorteile liegen in der kürzeren Bearbeitungszeit (TAT = turn around time), bedingt durch:

Wegfall der Transportzeit

Probenvorbereitung

Messung direkt am Patienten

Messung aus Vollblut 
Vorteile einer Vernetzung liegen für den POCT-Anwender darin, dass Messergebnisse gespeichert und dem Patienten automatisch zugeordnet werden, d.h. Integration in einen kumulativen Befundbericht.

Es ist nachvollziehbar wer die Patientenmessung und die QC -Messung durchgeführt hat. Die QC - Messungen pro Gerät sind automatisch dokumentiert und der Zeitpunkt für die nächste QC wird rechtzeitig angezeigt. Eine statistische Auswertung ist jederzeit möglich.

\title{
Liquid profiling in plasma: therapy-relevant tumor diagnostics in an interdisciplinary contex
}

\section{V15 - Talk Baraniskin}

\author{
“Liquid profiling as integrative part of clinical decision-making and for challenge-rechallenge concepts" \\ Alexander Baraniskin \\ Uni Bochum, Knappschaftskrankenhaus, Klinische Leitung, Bochum, Germany
}

"Colorectal cancer (CRC) is one of the most prevalent cancers and a leading cause of cancer mortality worldwide. The antiepidermal growth factor receptor (anti-EGFR) monoclonal antibodies cetuximab and panitumumab have improved clinical outcomes for patients with all wildtype RAS metastatic colorectal cancer ( $\mathrm{mCRC}$ ) and are part of standard chemotherapy for these patients, in particular for patients with leftsided mCRC. The exclusion of RAS mutations prior to therapy is mandatory for application of anti-EGFR antibodies.

Liquid biopsy, a noninvasive blood-based RAS mutation analysis of cell-free circulating tumor DNA (ctDNA), has been reported as a suitable method for mutational analysis in patients with mCRC. In contrast to tissue based RAS status determination, liquid biopsy is able to address tumor heterogeneity and treatment-related dynamic changes of molecular profiles.

Due to the intrinsic molecular heterogeneity of cancer, the RAS mutation status of primary tumors and metastases differs in about $20 \%$. The sampling bias caused by tissue heterogeneity may be overcome by the liquid biopsy.

The dynamical adaption of the CRC genome towards application of targeted drugs like anti-EGFR antibodies leads to acquired (secondary) resistance. To monitor this therapy-relevant phenomenon of clonal selection, the mutational profiles of patients with CRC should be continually tracked during the course of treatment. The new-onset of plasma DNA mutant fragments as a result of a clonal selection is a surrogate marker for disease progression. The mutation load decreases after the discontinuation of the therapy with EGFR-specific antibodies. This knowledge opens the perspectives for the anti-EGFR rechallenge. Accordingly, liquid biopsy plays a crucial role in determination of the appropriate point of time for initiation of the rechallenge therapy."

\section{Hämostaseologie}

\section{V16 - Talk Greinacher}

\section{Anti-PF4/Polyanion antibody induced autoimmune HIT: A new mechanism of autoimmunity}

\author{
Andreas Greinacher \\ Universitätsklinikum Greifswald, Institut f. Immunologie und Transfusionsmedizin/ Abteilung Trabsfusionsmedizin, Greifswald, Germany
}

Platelets contribute to innate immunity. We recently introduced the concept that hereby platelet factor 4 (PF4) plays an important role. Antibodies recognizing a neoepitope on PF4/polyanion (P) complexes opsonize bacteria via binding to PF4 bound to bacterial surface polyanions. When this bacterial host defense mechanism is misdirected to PF4/heparin complexes on platelets and monocytes, the prothrombotic adverse drug reaction heparin-induced thrombocytopenia (HIT) occurs.

HIT as a model for autoimmunity: HIT occurs when anti-PF4/P-antibodies activate PF4/heparin-coated platelets; while autoimmune-HIT occurs in the absence of heparin treatment. We assessed binding characteristics of different anti-PF4/polyanion antibodies by single molecule force spectroscopy. Anti-PF4/P-antibodies activating platelets only in the presence of polyanions show binding forces to PF4/P-complexes $<60 \mathrm{pN}$; antibodies activating platelets in the absence of polyanions (autoimmune-HIT antibodies) show much higher binding forces $\sim 100 \mathrm{pN}$. Most interestingly, these anti-PF4-autoantibodies substitute for heparin. They bind to PF4 alone and thereby induce the same change in the conformation of PF4 as heparin does. This induces exposure of the HIT neo-epitope, and recruits physiologic, polyanion-dependent anti-PF4/P-antibodies into the autoimmune-HIT process, resulting in massive, heparin-independent platelet activation. Antibody-mediated changes in endogenous proteins triggering binding of otherwise non-pathogenic antibodies may also be important for other antibody-mediated autoimmune disorders.

In conclusion, we show that PF4/P antibodies mediate an innate bacterial defense mechanism and provide a hypothesis why recent infections are often linked with autoimmune disorders. 


\title{
V17 - Talk Denis
}

\section{Von Willebrand disease type 2B: an ever surprising condition}

\author{
Cecile Denis \\ Le Kremlin-Bicetre cedex, France
}

Von Willebrand disease type 2B (VWD type-2B) is caused by so-called gain-of-function mutations, clustered in exon 28 of von Willebrand factor (VWF) gene and results in spontaneous binding to platelet glycoprotein (GP)Ib. In a counter-intuitive manner and despite these gainof-function mutations, VWD-type 2B leads to a bleeding disorder rather than a prothrombotic phenotype. Bleeding tendency is associated with heterogeneous clinical manifestations such as the decrease of high molecular weight-VWF multimers, presence of giant platelets and/ or platelet aggregates, and moderate to severe thrombocytopenia. One of the major predictor of the bleeding risk in VWD-type 2B patients is the severity of the thrombocytopenia.

In the past few years, we have extensively studied VWD-type 2B using patients material as well as in vitro and in vivo models focusing on one specific mutation, p.V1316M which leads to one of the most severe phenotype among VWD-type 2B mutations. We have uncovered new and unexpected mechanisms underlying the thrombocytopenia and described a platelet function defect unreported before.

Indeed, we found that the p.V1316M mutation in VWF led to altered platelet aggregation, spreading and thrombus growth, resulting from a lack of activation of the $\square \mathrm{IIb} \square 3$ integrin. As for the thrombocytopenia, we have accumulated evidence for both a central production defect with an actin cytoskeleton dysregulation as well as a an increased clearance of platelets from the circulation due to an increased uptake of VWF-type 2B/platelet complexes by macrophages.

Our results highlight the complexity of VWD-type 2B and may be of importance for the treatment of the patients. Indeed, strategies aiming to restore a normal platelet count such as thrombopoietin receptor agonists or LIM kinase inhibitors may be beneficial in some patients with VWD-type 2B.

\section{Präanalytik in der Pädiatrie}

\section{V18 - Talk Kulikowski}

\section{Präanalytische Phase: Tipps und Tricks für das Labor}

Ines Kulikowski

Fa. Sarstedt, Germany

The preanalytical phase is an important aspect in the determination of valid results in laboratory medicine. These can only be ensured by thoroughly considering all influencing variables and interfering factors. This lecture has its focus on the preanalytical pitfalls in the lab. Of paramount importance is the optimisation of the centrifugation conditions, a process analysis in case of haemolytic samples as well as the possibilities offered by preanalytical consulting.

\section{V19 - Talk Duppel}

\section{Von der Materialgewinnung bis hin zum Laborbefund - eine Herausforderung für alle Beteiligten}

\author{
Uta Duppel \\ Regensburg, Germany
}

Pediatric blood sampling for laboratory testing is a challenge for any person involved - for children, parents, nurses and doctors but also for laboratory staff, who have to deal with little amounts of blood and are expected to produce valid blood values.

Due to the special anatomy, physiology as well as the psychological and emotional condition of pediatric patients blood sampling has to be planned carefully and the necessity of every single laboratory testing has to be critically questioned. Good communication between clinical and laboratory staff can help to optimize this process.

Besides the painful and distressful aspect of capillary, venous and arterial blood collection repetitive laboratory analyses can cause a relevant blood loss leading to anemia especially in preterm infants. The smaller anatomical proportions require particular blood drawing techniques which differ significantly from those applied to adults. However, these techniques bear the risk of producing false laboratory results. Physicians as well as laboratory staff have to be aware of these potential pitfalls when interpreting laboratory values. 
Of course, these aspects also have to be considered in sample collection of other body fluids such as urine or cerebrospinal fluid or in taking smears.

\title{
M5 Mikroskopiekurs
}

\section{V20 - Talk Schuff-Werner, Dreißiger \\ Digitale versus konventionelle Mikroskopie im hämatologischen Labor}

\author{
Peter Schuff-Werner'; Katrin Dreißiger ${ }^{2}$ \\ ${ }^{1} M D I$ Laboratorien GmbH Medizinisches Versorgungszentrum, Labormedizin, Berlin, Germany; ${ }^{2}$ Universitätsmedizin Rostock, Institut für \\ Klinische Chemie und Laboratoriumsmedizin, Rostock, Germany
}

Microscopic white blood cell (WBC) differentiation was introduced in the haematological diagnostic laboratory when appropriate cell dyes became available. With the invention of automated cell counting based on impedance technology by Wallace Coulter, it was possible to differentiate cells based on their size (volume) and improved technologies allowed a 5 parameter differential blood count.

Today, even more sophisticated analyses of physical (conductivity), optical signals (light scatter) from intact and from cells with selectively lysed cell membrane and the use of fluorescence staining allow the automated differentiation of normal and pathological cells, e.g. blasts and NRBC. But despite these compelling technological advances, the conventional microscopic differentiation of $100 \mathrm{WBC}$ on a dyed blood smear remained the gold standard in the hematological laboratory, although this approach is time consuming, personal binding and therefore expensive.

The development of pattern recognition systems based on military technologies used in Cruise Missiles, did not find a real input into the complete blood count (CBC) diagnostics.

Therefore it was a real step forward when advanced computer technology with high storage capacities allowed the development of the socalled digital microscopy. In the meanwhile this technology is increasingly spreading in hematological routine laboratories.

The aim of the offered course is to actively compare conventional WBC differentiation with CBCs made by the Cellavision Diffmaster. For this purpose selected microscopic slides from reactive and pathological blood samples from children and adults will be actively differentiated by the course participants and then compared to CBC made by digital microscopy. The advantages and disadvantages of the new technology will finally be discussed.

\section{Therapeutisches Drug Monitoring heute: Mehr als nur Pharmakokinetik}

\section{V21 - Talk Alffenaar}

\author{
Pharmacodynamics meets pharmacokinetics: State of the art TDM of antibiotics (Pharmakodynamik trifft \\ Pharmakokinetik: State of the Art TDM von Antibiotika)
}

\author{
Jan-Willem Alffenaar \\ University Medical Center Groningen, Clinical Pharmacy and Pharmacology, Groningen, Netherlands
}

With increasing pathogen resistance dose optimization of anti-microbial drugs should be a priority.

Therapeutic drug monitoring can help to assure adequate drug exposure at the individual patient level. To be able to integrate pharmacokinetic (PK) and pharmacodynamics (PD) knowledge to optimise drug therapy information about the susceptibility of the pathogen is needed in addition to the measured drug concentration. Based on this information one can adjust the dose to attain the PK/PD target. The PK/ $\mathrm{PD}$ target is often established on data from in vitro and in vivo dose fractionation studies. Despite achieving the optimal PK/PD target in a patient this does not necessarily mean that the patient will respond to treatment. Mixed infections, difficult to penetrate site of infections, or failing immune system may underlying reasons why therapy could fail. In addition to drug exposure and information about the pathogen a measure response would be welcome. Traditionally body temperature and C-reactive protein have been used as short term measure of response. Unfortunately these measures are often not very sensitive or specific. Introduction of for example gallactomannan, $\beta$-D glucan, procalcitonin provide an measure of response that can be used in addition to the measured drug concentration and pathogen susceptibility. The presentation will provide background information on pharmacokinetic and pharmacodynamics parameters and provide a frame work how they can be integrated to provide the patient with best possible care. 


\title{
V22 - Talk von Ahsen
}

\section{PharGKB, text books and UpToDate databases as tools for pharmacogenetic information: TPMT as example}

\author{
Nicolas von Ahsen \\ Universitätsmedizin Göttingen, Abt. Klinische Chemie, Göttingen, Germany
}

An example is taken how to gather relevant pharmacogenetic information from a website resource, current pharmacology textbooks and the evidence-based clinical decision system UpToDate. PharmGKB is a curated webpage hosting pharmacogenetic information. They are closely related to the CPIC summaries of pharmacogenetically relevant genes. The presentation leads through the case of a classical pharmacogenetic polymorphism in TPMT of relevance to thiopurine treatment. PharmGKB lists TPMT as a very important pharmacogene so that an individual summary with review character is offered. The website further bundles the genetic information about the relevant SNPs in TPMT and their clinical implications including dose adjustments based on CPIC and other guidelines. FDA prescribing information and other drug label sources are available. The metabolic pathways of thiopurine drugs are shown in somewhat overloaded accuracy. Those interested in current scientific literature will find references to TPMT pharmacogenetic papers. Other sources are the 2018 editions of Katzung’s Basic \& Clinical Pharmacology, 14th ed., McGraw-Hill and Goodman and Gilman's Pharmacological Basis of Therapeutics, 13th ed, McGraw-Hill. The Katzung gives a very concise outline of TPMT pharmacogenetics and dosing recommendations. Information in Goodman and Gilman's book are more distributed and closely related to the labeling information. The UpToDate database (paid subscription) gives an authoritative and still concise summary on TPMT pharmacogenetics. The scope is widened to common clinical problems and how to handle them. However, the recommendations differ for rheumatic disease (not in favor) and inflammatory bowel disease (in favor of TPMT monitoring). My personal preference for one-stop gathering of relevant pharmacogenetic information is in the order UpToDate > Katzung > PharmGBK / CPIC reviews > Goodman and Gilman.

\section{V23 - Talk Widmer}

\section{TDM of cancer therapy: An emerging field}

\author{
PD Dr. Nicolas Widmer \\ Service of Clinical Pharmacology, Lausanne University Hospital, Lausanne, Switzerland
}

\begin{abstract}
The therapeutic use of some drugs could be optimized by an individualization of their dosage regimen, based on blood or plasma circulating concentrations measurement in patients thanks to the therapeutic drug monitoring (TDM) feedback strategy. The modern oral targeted anticancer drugs are directed against cancer-specific molecules and signaling pathways and seems to be characterized by impressive efficacy and limited non-specific toxicities. Nevertheless, drug resistance, persistence of cancer stem cells, and adverse drug effects still limit their ability to stabilize or cure malignant diseases in the long term.

Oral anticancer drugs are generally licensed for use at fixed doses even though most of them would meet almost all criteria for successful TDM implementation in clinical practice: long-term and continuous therapy, availability of appropriate bioanalytical methods for quantification in clinical samples, high inter-individual pharmacokinetic variability (because of variations in metabolism, protein binding, etc.) but limited intra-individual pharmacokinetic variability, alongside consistent associations between concentration and response. They have also the potential to be involved in multiple interactions (drug-drug; drug-food) and exhibit adherence issues with lifelong treatment. Imatinib was the first to benefit from prospective randomized controlled trial to assess the clinical benefit of a TDM approach, however with contrasting results. Progresses in TDM are still needed, especially for new targeted anticancer agents, both on a conceptual and a practical level. Advances toward modern TDM approaches are ongoing in the field of oncology and other therapeutic area: development of appropriate pharmacokinetic and pharmacokinetic/pharmacodynamic models and improvement of meta-analysis methods to aggregate them, validation of therapeutic target in randomized controlled trials, elaboration of conceptual and practical framework for practitioners and conduct of technological efforts toward miniaturization and development of computer software to assist them.
\end{abstract}

\section{Immunohematology}

\section{V24 - Talk Wagner}

\section{Genomic Rhesus testing, what have we learned?}

\section{Franz Wagner}

DRK-Blutspendedienst NSTOB gemeinnützige GmbH, Hauptabteilung Spender- und Qualitätskontrollabor, Springe, Germany

The antigens of the RH blood group system are encoded by two highly homologous genes, RHD and RHCE that face each other with their 3' ends. The coding sequence of RHD is dispersed over 56374 bp. Antigen D is encoded by RHD, antigens C, c, E and e by RHCE. The most 
frequent D-negative haplotype is caused by a deletion of RHD. Gene conversions between RHD and RHCE typically lead to RHD-CE-D (or analogous RHCE-D-CE) double hybrid alleles that in case of RHD often express partial or no D antigen. Missense mutations in exofacial protein segments usually incur a partial D phenotype, those in non-exofacial protein segments often a weak D phenotype. Other mechanisms of weakened D antigen expression are splice site mutations or deletions / duplications. Frameshift mutations and stop codons may cause a D negative phenotype. Molecular prediction of the phenotype is hampered by the homology of the proteins, for example the nucleotides causing C antigen expression in RHCE are surrounded by an RHD-like segment of about 5000 bp. Nowadays, more than 500 RHD alleles are known. Different alleles express different phenotypes with different clinical behavior; the molecular characterization of a variant phenotype is far more informative than a serologic description. For example, more than 150 alleles with weak D phenotype are known; for three of these, accrued data indicate that allo-anti-D production is almost impossible, while other alleles with largely similar phenotype are known to be associated with allo-anti-D. As a result, the current guidelines of several countries advocate molecular determination of the RHD allele in patients with weak expression of antigen D and suggest a RhD-positive transfusion strategy for weak D type 1, type 2 and type 3 but not for other RHD alleles. Currently, molecular testing is mostly based on the detection of one or multiple SNP. Whole exome sequencing or whole RHD gene sequencing is likely to improve Rhesus antigen prediction but not yet in widespread use. The availability of whole genome data for different RH haplotypes will increase the understanding of the phylogenetic relationship of the different RH haplotypes.

\title{
V25 - Talk Lejon Crottet
}

\section{Daratumumab treatment for multiple myeloma interferes with routine blood bank tests - what is the best solution?}

Sofia Lejon Crottet

Reference Laboratory Swiss Transfusion SRC, Immunohematology, Bern, Switzerland

In 2016 Daratumumab (DARA) was approved in Europe as a third-line treatment for patients with multiple myeloma (MM). DARA is an IgG1k human monoclonal antibody targeting the cell-surface glycoprotein CD38, which is highly expressed on the surface of MM cells. CD38 is also expressed on other cells, such as erythrocytes, and thus causes panreactions when DARA containing plasma is used in the indirect antiglobulin test (IAT). Consequently, DARA will interfere with the antibody screening, antibody identification as well as the cross-match and mask detection of potential allo-antibodies to erythroid antigens. DARA will not however interfere with the standard identification of ABO/RhD antigens.

In order to eliminate the panreactivity caused by DARA, test cells are treated with dithiothreitol (DTT). This reducing agent disrupts the disulphide bonds in the CD38 molecule, thereby inhibiting the DARA binding. Unfortunately, DTT destroys also important blood group antigens such as KEL1, KEL3, DO1 and DO2. To reduce the allo-immunization risk, it is recommended in Switzerland to transfuse Rh, K and Kp(a) compatible blood units to patients undergoing a treatment with DARA.

Furthermore, the DARA interference can be reduced by enzymatic treatment of the test cells with enzymes such as papain and trypsin, which however also destroy certain important blood group antigens.

Several other methods such as the use of umbilical cord red blood cells, soluble CD38 proteins and antibodies against DARA have been proposed. Recently, a new reagent, DaraEx (Inno-Train), became available on the market that inhibits the reactions between DARA and the test cells without negatively influencing other blood group antigens. This interesting approach has the capability to increase the safety of the pre-transfusion testing.

For routine laboratories, not only the time aspect is important, but also the costs. Each laboratory will have to consider all factors when choosing their method of choice for eliminating the DARA interference in pre-transfusion testing.

\section{Symposium Precision Medicine bei chronisch-inflammatorischen Erkrankungen}

\section{V26 - Talk Renz}

\author{
Asthma - eine heterogene Erkrankung: Endotypen, Biomarker, therapeutische Targets \\ Harald Renz \\ Philipps Universität Marburg, Institut für Laboratoriumsmedizin, Marburg, Germany
}

Asthma is one of the most prevalent chronic inflammatory lung diseases affecting approximately 300 million individuals worldwide. Heterogeneity occurs on several levels, including the underlying chronic inflammatory response. The best characterized inflammatory response 
pattern is termed type-2 inflammation. This refers to a dominance of T-helper (Th)-2-cells with respective downstream events on the molecular and cellular level. Th-2-cytokines are not only produced by CD4+ T-cells but also by specialized innate lymphocytes (ILCs) and other cell population. The cytokine pattern is characterized by increased levels of IL-4, IL-5 and IL-13. These cytokines regulate many downstream events including the biology of eosinophils, mass-cell activation, epithelial responses and the production of IgE by B cells. Based on this paradigm patients suffering from type-2 inflammation should potentially benefit from targeted therapies aiming to block defined molecules in this regulatory network. Such strategies have been developed using biologicals (monoclonal antibodies) directed against one or several of these cytokines or cytokine receptors. However, the development of type-2 inflammation depends on the expression of transcription factors which are necessary to direct T-cell specialization and maintenance of already specialized T-cells. For type-2 inflammation it is very well established that the transcription factor GATA-3 is sufficient and essential for the development of type-2 responses. GATA-3 is not only expressed in CD4 Th-2 cells, but also in ILC-2 cells, mast cells, eosinophils and epithelial cells. Therefore, GATA-3 plays an essential role in type-2 inflammation. However, in order to therapeutically target this transcription factor an intervention strategy is required which is able to block GATA-3 on the intracellular level, since GATA-3 is not secreted. We developed a highly specific antisense molecule for GATA-3 messenger (m) RNA which blocks the translation of GATA-3 mRNA into GATA-3 protein. Our antisense molecule (SB010) has, in addition to its specific binding activity to the GATA-3 mRNA, an additional cleavage activity due to an intrinsic catalytic domain of SB010 which cuts GATA-3 messenger RNA in at least two fragments. Following extensive preclinical analysis, toxicology studies and three clinical safety studies in healthy and asthmatic individuals, a phase IIa proof-of-concept clinical trial was performed in mild asthmatic patients. Treatment was carried out for 28 days by daily inhalation of a single dose of $10 \mathrm{mg}$ SB010. Allergen provocations were performed before and after the treatment cause. A primary endpoint of this study, significant reduction of the late phase asthmatic response, was reached, and in addition, also significant effect on the early phase allergic response was measured. The degree of improvement could be furthermore related to the level of blood eosinophils before treatment. These data indicate for the first time that transcription factors represent potential targets for pharmacotherapy in asthma. However, additional clinical trials are warranted to firmly establish this new treatment modality.

\title{
V27 - Talk Müller-Ladner
}

\section{Präzisionsmedizin der Rheumatologie}

\author{
Ulf Müller-Ladner \\ Campus Kerckhoff, Bad Nauheim, Justus-Liebig-Universität Gießen, Rheumatologie und Klinische Immunologie, Bad Nauheim, Germany
}

In the past two decades, several new drugs and treatment strategies have been introduced into the field of rheumatic and systemic inflammatory diseases. Herewith, many patients can achieve remission or low disease activity if the disease or entity is diagnosed early and treated as quick as possible. However, a substantial number of individuals do not respond adequately to treatment. Therefore, reliable biomarkers or other (clinical) criteria are urgently needed to avoid delayed effective therapy time. In addition, tight monitoring and control can increase the likelihood of remission in these patients, providing the basis for targeted therapy and precision medicine. The current concepts include a personalized approach based primarily on the individual risk stratification and, if applicable, on the genetic background of the patient, e.g. distinct HLA phenotypes. To further realize the purpose of personalized medicine in rheumatology, the currently most useful examples of biomarkers capable of identifying and predicting the outcome of patients, to set appropriate preventative measures, is represented by diseasespecific serum autoantibodies. Aside the long-known rheumatoid factors and the more specific antibodies against citrullinated peptides in arthritides, many more autoantibodies are being established for other systemic diseases such as vasculitides. Prediction of effect can also be dependent on measurement of anti-drug antibody levels. However, the high variability of the different disease entities usually reuqires more complex approaches with multi-parameter measurements and, still in the preclinical validation phase, multigen arrays. Taken togehter, the ultimative goal, to provide the right drug for an individual patient at the right time is a fascinating goal with many problems to be solved, especially the correct handling of big data.

\section{Das Darmmikrobiom}

\section{V28 - Talk Fricke}

\section{Dysbiosis - what makes the microbiome 'good' or 'bad'?}

\author{
Florian W. Fricke \\ University of Hohenheim, Institute of Biological Chemistry and Nutrition (140d), Stuttgart, Germany
}

The human microbiota, i.e. the sum of all microbial life in and on the human body, continues to create tremendous excitement within and beyond the scientific community. Numerous immunological, infectious and metabolic diseases and disorders have been associated with specific features of the gastrointestinal (GI) microbiota, such as microbial diversity or the presence and absence of specific bacterial groups. 
Dysbiosis is a commonly used but not well-defined term to describe this general state of microbiome disturbance. Still, the reported associations between the microbiota and diseases are mostly correlative and many studies have not been carefully controlled for confounding factors. Moreover, little is known about the mechanisms that establish, maintain or modulate the GI tract microbiota, leaving few options for microbiota-targeted therapy.

We used 16S rRNA gene amplicon sequencing and metagenomics to study the microbiota at different locations along the GI tract using animal and human samples. We highlight current problems in the field and provide new insights into (i) the composition and structure of the stomach microbiota as a potential bottleneck between the upper and lower GI tract; (ii) the characteristics of microbial dysbiosis and strain transfer during Clostridium difficile infection and reestablishment of microbial homeostasis during fecal microbiota transplantation (FMT); and (iii) the identification and evaluation of novel biomarkers to functionally describe the microbiota in the context of host-microbe interactions.

\title{
V29 - Talk Hiergeist
}

\section{Challenges for the Clinical Implementation of Microbiome Sequencing}

\author{
Andreas Hiergeist \\ Universitätsklinikum Regensburg, Institut für Med. Mikrobiologie und Hygiene, Regensburg, Germany
}

Rapid advances of high throughput sequencing based technologies have opened up entirely new strategies to extensively characterize the microbial ecology of human body habitats independent of laboratory cultivation. Several large-scale seminal studies have revealed that various human diseases are closely associated with compositional changes of the intestinal microbiota. However, the causal connection between these microbial imbalances and clinical pictures as well as the underlying pathophysiological mechanisms of microbial host interactions are still extensively unknown for many pathologies. Clinical application of microbiome analysis requires a diligent implementation of quality controlled standardized wet lab and bioinformatic protocols as well as continuous quality monitoring and accreditation besides conducting well-controlled cohort studies. If these prerequisites are met, microbiome analyses have the potential to be successfully applied in clinical practice as a prognostic tool, as well as for the development of individualized therapies.

\section{Poster}

\section{Posterwalk Pädiatrische Labormedizin/Leitlinien und diagnostische Pfade}

\section{DGKL-P001}

\author{
Aldosterone, cortisol and cortisone plasma levels in infants ( $<1$ year of age) measured with LC-MS/MS \\ Yoon Ju Bae ${ }^{1}$; Robert Zeidler ${ }^{1}$; Ronny Baber ${ }^{1}$; Alexander Gaudl ${ }^{1}$; Mandy Vogel ; Uta Ceglarek ${ }^{1}$; Wieland Kiess ${ }^{3}$; Antje Körner ${ }^{3}$; Joachim Thiery'; \\ Jürgen Kratzsch ${ }^{1}$ \\ ${ }^{1}$ Universitätsklinikum Leipzig, Institut für Laboratoriumsmedizin, Klinische Chemie und Molekulare Diagnostik, Leipzig, Germany; ${ }^{2}$ University \\ of Leipzig, Leipzig Research Center for Civilization Diseases - LIFE, Leipzig,; ${ }^{3}$ University of Leipzig, Hospital for Children and Adolescents and \\ Center for Pediatric Research, Leipzig
}

Background: Infants ( < 1year) show elevated aldosterone plasma levels. The insensitivity of mineralocorticoid receptors (MR) in the immature kidney and perinatal osmotic changes are discussed. In addition, the binding of cortisol to MR could contribute to the aldosterone elevation. We hypothesized that an enhanced metabolism of cortisol to cortisone could be a compensatory mechanism to avoid further aldosterone elevation in infants. To confirm this suggestion we analyzed aldosterone, cortisol and cortisone levels in infants and young children.

Methods: Healthy infants $(\mathrm{n}=286, \mathrm{~m} / \mathrm{f}=127 / 159, \leq 1$ year) were compared with children $(\mathrm{n}=356, \mathrm{~m} / \mathrm{f}=185$ / 176, 5 - 10 years) within the LIFE-Child cohort (Poullain A et. al. 2017). Blood was taken in the mornings. Aldosterone, cortisol, and cortisone were analyzed by LC-MS/ MS (Gaudl A et al, 2016).

Results: Aldosterone, cortisone, and cortisone/cortisol ratio were significantly higher in infants than in younger children ( $\mathrm{p}<0.0001)$. Agespecific reference intervals for aldosterone serum levels have been established by LC-MS/MS analytics: 3 to 6 months ( $\leq 3255$ pM $(90 \%$ CI: $2680-4890 \mathrm{pM}), \mathrm{N}=156), 6$ to 12 months ( $\leq 2706$ pM (90\% CI: $1570-3110 \mathrm{pM}), \mathrm{N}=127)$. Cortisol was not significantly different between the two groups.

Conclusion: We present for the first time with LC-MS/MS that plasma aldosterone and cortisone levels are elevated during the first year of life. The increased levels of plasma cortisone indicate a remaining prenatal protective mechanism against excessive maternal cortisol levels. The enhanced inactivation of cortisol to cortisone may be a compensatory mechanism to avoid further aldosterone elevation in infants. 


\title{
DGKL-P002
}

\section{Adopting Age-Dependent immature granulocytes (IG\%) Reference Ranges in Paediatrics}

\author{
Jürgen Christoph \\ Auf der Bult - Kinder- und Jugendkrankenhaus, Labor, Hannover, Germany
}

\begin{abstract}
Aims: Verification of pediatric reference ranges of immature granulocytes (IG\%), measured on the hematology system Sysmex XN-350. Verifying of published reference ranges with at least 20 healthy children in each of our 15 (paediatric) age groups is expensive, a validation with more than 120 healty children in each age group would be unrealistic.

Methods: The percentiles of the IG\% and the sum of promyelocytes, myelocytes and metamyelocytes were calculated from 22,110 blood counts in the period from 25.02.2016 to 25.05.2018 with both fluorescence flow cytometry measurement of IG\% and microscopic differentiation, furthermore the differences Delta-P between the respective percentiles P1, P3, P5, P10, P25, P50 (median), P75, P90, P95, P97, P97.5, P98, P99. IG\%-Reference ranges were calculated using the Trillium Normalizer.

Results: Only on birthday (day 0: Delta-P75 1.40, Delta-P90 1.39), age 4-7 days (Delta-P95 2.27) and 7-14 days (Delta-P50 1.1) and in the small group of adults (age $>18$ years, $\mathrm{N}=181$; delta-P95 1,1) differences between percentile values were $>1$. For children between the ages of 1 to 18 years, the mean of the IG\%-P90 is 0.60 and the IG\%-P95 is 1.12. On birthday (day 0, mean age about 12 hours) our IG\% correspond to those reported from Cleveland (OH, USA; Acta Paediatr. 2014 May;103(5):494-7): P32 1.0 (Cleveland P30), P47 1.5\%, P53-P54 1.75\%.

The upper IG\% reference range limit calculated with the Trillium Normalizer is 2.5 at the age of 15 to 30 days, 1.4 from 1 to $<3$ months, 1 for 3 month to $<2$ years, 0.8 for 2 to $<6$ years, 0.7 for 6 to $<15$ years and 0.6 from 15 to $<18$ years.

Summary: Small percentile differences between flow cytometric IG $\%$ and microscopic differentiation and good agreement of percentiles in the critical age group of birthday with literature data allow a verified adopting of published IG\% reference ranges. A direct pairwise comparison between manual microscopic results and automatic counting would have limitations (according to Rümke) due to rather wide 95\% confidence intervals of 100 white blood cells (WBC) differential microscopy in routine clinical practice.
\end{abstract}

\section{DGKL-P003}

\section{Development and design of an illustrated quick reference guide concerning blood drawing for coagulation testing for health professionals in Tyrol}

Miriam Bachlechner'; Heidi Oberhauser ${ }^{1}$

${ }^{1}$ Fhg- Zentrum für Gesundheitsberufe Tirol GmbH, Bachelorstudiengang Biomedizinische Analytik, Innsbruck, Austria

Keywords: illustrated guideline, practice innovation, simplify application, blood drawing

Background: Various studies prove that pre- analytical errors are the main cause for errors in laboratory diagnostics. The data vary between $46 \%$ and $68,2 \%$ [1]. Errors in laboratory testing do not only increase healthcare expenses, in about $25 \%$ of the cases, they also may have direct consequences to the patients [2]. Blood drawing is mostly performed outside the laboratory, like in diverse hospital wards or medical practices and from different health professionals. For this reason the knowledge about the correct blood sampling is of utmost importance. Furthermore, partly contradictory statements concerning blood drawing for coagulation testing, especially regarding filling level, order of drawing and usage of discard tubes, complicate the blood drawing process additionally [3]. There is evidence, that knowledge and awareness of blood drawing recommendations are lacking among health care professionals outside the laboratory [4].

Central issue: The aim of this study is first, to standardise the blood drawing procedure for coagulation testing in Tyrol's hospitals and educational institutes for health professionals. In second, for an easier application, to design an illustrated and practicable quick reference guide, which is easy to conceive and to implement.

Methods: Via online-questionnaire, teachers and students of different educational institutes for health professionals in Tyrol were asked about instructed regulations concerning blood drawing for coagulation testing. Hospital ward- and laboratory manager were asked about blood drawing sets, recommendations used and groups of health professionals who are carrying out the blood drawing. In particular, laboratories were also consulted about criteria and percentage of refusal of samples because of inadequate filling level.

Expected results: First results show, as expected, a slightly difference between educational institutes and hospitals relating to recommendations of blood drawing. With the active cooperation of the teachers, the results of the survey will be integrated into the education of health professionals to achieve a standardised teaching regarding to regulations concerning blood drawing for coagulation testing.

Summary: Correct blood drawing is an essential condition for proper laboratory results. Therefore, it is important, that all involved groups of health professionals will be informed about the process and possible consequences. In this condition, an illustrated quick reference guide supports the daily routine. Actively involving of apprentices and practitioners increase the implementation of evidence-based decisions into clinical routine. 


\title{
DGKL-P004
}

\author{
The Marburg Cerebrospinal Fluid Model reveals pathogenesis of diagnostic pathways of inflammations in human \\ central nervous system (CNS)
}

Tilmann Otto Kleine

Universitätsklinikum Gießen \& Marburg UKGM, Institut für Laboratoriumsmedizin \& Pathobiochemie, Molekulare Diagnostik, Referenzlabor für

Liquordiagnostik, Marburg, Germany

The Marburg Cerebrospinal Fluid Model reveals pathogenesis of diagnostic pathways of inflammations in human central nervous system (CNS) Tilmann O. Kleine Universitätsklinikum Giessen \& Marburg: Institut für Laboratoriumsmedizin \& Pathobiochemie, Molekulare Diagnostik; Referenzlabor für Liquordiagnostik; Baldingerstraße. 35043 Marburg, Germany

Objective: Laboratory medicine lays the basis for diagnosis and therapy of inflammations in human CNS, registered in quidelines and diagnostic pathways of acute meningitis / encephalitis, opportunistic CNS infections (toxoplasmosis, tuberculous / cryptococcal meningitis, infections with cytomegalovirus) and radicular syndromes with spinal nerves.

Pathogenesis of CNS inflammations is revealed with Marburg Cerebrospinal Fluid (CSF) Model which implies transfer of small blood lymphocytes of 7-8 $\mu \mathrm{m}$ diameters into ventricular CSF through leaky circum ventricular organs (CVOs); less transfer occurs with large lymphocytes / monocytes of 12-15 $\mu \mathrm{m}$ cell diameters. Reflux of lymph lymphocytes from ductus thoracicus into lumbar CSF is described in ref. 1.

Methods: Microscopic counting of leukocytes / lymphocytes starts CSF analysis: Ventricular, suboccipital, lumbar CSF samples are analyzed together with corresponding venous blood samples. Transient or primary bacteremia is detected with bacteria in human venous blood and CSF samples.

Results: Bacteremia is observed with Gram positive bacteria (Staphylococcus aureus of $<0.25 \mu \mathrm{m}$ width and $<1,5 \mu \mathrm{m}$ in length, streptococcal species of $<2.0 \mu \mathrm{m}$ in diameter and $<2.0 \mu \mathrm{m}$ in length, enterococci with ovoid forms of $\sim 2.5 \mu \mathrm{m}$ size) and Gram negative bacteria (E. coli of $<2 \mu \mathrm{m}$ in length and $\sim 0.5 \mu \mathrm{m}$ in width, klebsiella pneumoniae: straight rods of $<2 \mu \mathrm{m}$ width with thick capsule, salmonella with $<2.0 \mu \mathrm{m}$ rod size). Bacteremia is described as occult bacteremia with trivial illnesses of respiratory tract infection, fever, otitis media or diarrhea preceding CNS inflammations (ref. 2). Thus, transfer of bacteria from blood through leaky CVOs into ventricular CSF occurs like the transfer of blood lymphocytes into CSF. However, long bacteria impede rate of their transfer through CVOs into CSF. Cancer cells / lymphocytes are found in ductus thoracicus lymph (ref. 3) that refluxes into lumbar CSF to infiltrate spinal nerve roots; thus causing radicular nerve syndromes.

Summary: Bacteremia with trivial illnesses precedes inflammations of human CNS, since small bacteria pass leaky CVOs like larger blood lymphocytes to cause severe CNS inflammations. Reflux of thoracic lymph containing cancer cells and lymph lymphocytes causes radicular syndromes of spinal nerves.

No transfer of bacteria from blood through plexus choroidei into ventricular CSF occurs together with CSF formation through tight junctions in plexus epithelia.

Ref. 1: T.O. Kleine. Cytometry Part A 2015; 87A:227-243.

Ref. 2: Rosenberg N. Am J Emergency Medicine 1983; 2:231-237.

Ref 3: Schischmanov N. Nagoya J Med Sci 2013; 75:153-159.

\section{Posterwalk Mikrobiologie und Mikrobiom}

\section{DGKL-P005 / PV01}

\section{Rapid detection of multi-drug resistant Enterobacteriaceae by metabolic monitoring}

\author{
Maximilian Kittel ${ }^{\text {; }}$ Peter Findeisen ${ }^{1}$; Parviz Ahmad-Nejad²; Beniam Ghebremedhin²; Thomas Miethke ${ }^{3}$; Michael Neumaier \\ ${ }^{1}$ Medical Faculty Mannheim of Heidelberg University, Institute for Clinical Chemistry, Mannheim, Germany; ${ }^{2}$ HELIOS Klinikum Wuppertal, Witten/ \\ Herdecke University, Institute for Medical Laboratory Diagnostics, Center for Clinical and Translational Research, Witten, Germany; ${ }^{3}$ Medical \\ Faculty Mannheim of Heidelberg University, Institute for Medical Microbiology and Hygiene, Mannheim
}

Background: The increasing number of MRGN bacteria provides great challenges for the empiric antibiotic therapy. While bacterial identification has been greatly accelerated by the introduction of MALDI-TOF-MS, the antibiotic susceptibility testing (AST) remains time-consuming. Methods: Gram-negative isolates (E. coli and Klebsiella spp.) were directly extracted and identified from positive blood cultures using MALDITOF-MS. Bacteria were incubated in glucose-supplemented broths each containing one of the MRGN defining key antibiotics (Ceftazidime, Piperacillin, Imipenem, and Ciprofloxacin). The dynamics of glucose utilization under the influence of antibiotics in bacterial suspensions designated liquid AST (L-AST) were quantitatively measured to determine susceptibility.

Results: L-AST can be run on routine clinical chemistry analyzers and easily integrated into laboratory routines. L-AST yields critical resistance information within 90-150 min. downstream of a positive MS-based identification. The results showed a high concordance with routine susceptibility testing for 168 assessed isolates. Analysis of turnaround time for 42 clinical samples indicated that L-AST results were in mean $34 \mathrm{~h}$ earlier available than routine AST results. 
Conclusion: As exemplified for E. coli and Klebsiella species, L-AST provides substantial acceleration of bacterial resistance testing following standard MALDI-TOF-MS identification. The assay is a simple and low-cost method that can be integrated into every clinical laboratory to allow for 24/7 AST. This approach could enable improved clinical decision-making of patients diagnosed with Gram-negative sepsis caused by E.coli or Klebsiella ssp.

\title{
DGKL-P006
}

\section{Various Escherichia coli pathotypes adhere differentially to glycoprotein 2 of human, bovine and porcine origin}

Christin Bartlitz'; Rafat Kolenda²; Jonas Noack; Peter Schierack $^{3}$

${ }^{1}$ BTU Cottbus - Senftenberg, Senftenberg, Germany; ${ }^{2}$ Wroctaw University of Environmental and Life Sciences, Department of Biochemistry and Molecular Biology, Wrocław,; ${ }^{3}$ BTU Cottbus - Senftenberg, Biotechnologie, Senftenberg

Background: The glycoprotein 2 (GP2) is a membrane-anchored protein that is mainly expressed in the pancreas, as well as on cells in various mucous glands in the digestive, respiratory and genital tracts. GP2 takes part in binding of type 1 fimbriae (T1F)-positive bacteria. T1F are adhesive organelles of commensal and pathogenic bacteria such as E. coli, and its fimbrial tip protein FimH interacts with glycoproteins. This binding causes an immune response, including autoimmunity against GP2. According to several studies, GP2 is involved in inflammatory bowel diseases such as Crohn's disease and it was identified as an autoantigen of pancreatic antibodies.

The question: GP2 gene orthologs can be found among mammals. Depending on animal, various number of alternative splice variants of GP2 are expressed, for instance four isoforms have been found in human and two in pig and cattle. However, the binding of E. coli to GP2 of different mammal species has not yet been compared. Therefore our goal was to investigate this interaction.

Material and methods: First, human, porcine and bovine GP2 isoforms (for each two) were expressed with the baculovirus expression system. SF9 insect cells were infected with baculoviruses (containing the GP2 sequences) and then expressed the protein. GP2 isoforms were purified on gravity flow Ni-NTA resin columns and their purity was evaluated with silver staining. Next, GP2 assays with the purified GP2 were performed for the investigation of bacterial binding. In this study, 96-well plates were coated with GP2 and then incubated for $2 \mathrm{~h}$ with 60 commensal, 60 enteropathogenic (EPEC) and 60 enterotoxigenic E. coli (ETEC) of human, porcine and bovine origin. The bound E. coli were fixed with paraformaldehyde and stained with propidium iodide. Additionally, DAPI beads were added for focusing. The images were taken by the imaging system Aklides and the bacteria were count with the analysis software MaxiSlider.

Results: The SF9 insect cells were able to express large amounts of GP2. The purification process with Ni-NTA resin columns yielded wellpurified proteins. The results of the GP2 assay showed large differences in bacterial binding. However, we did not see single isolates or groups which specifically bound to a GP2 isoform. If an isolate was well bound to a GP2 isoform then it also bound others equally well. In contrast, we were able to observe differences in adhesion properties of tested pathotypes, for example porcine EPEC adhered best and porcine ETEC adhered worst to GP2. 43 isolates did not bind to any GP2 isoform.

Conclusion: The bacterial binding behaviour differs significantly among tested isolates. In future, it will be important to investigate other factors that can influence the binding behaviour of E. coli to GP2, for example the influence of FimH expression levels and single nucleotide polymorphism in FimH gene of E. coli on GP2 binding.

\section{Posterwalk Mikrobiologie und Mikrobiom}

\section{DGKL-P007}

\author{
Development of reference materials for methicillin-resistance in Staphylococcus aureus: feasibility, homogeneity and \\ stability assessment
}

Beniam Ghebremedhin ${ }^{1}$; Parviz Ahmad-Nejad

${ }^{1}$ Helios University Clinic Wuppertal - Witten/Herdecke Univ., Centre for Clinical and Translational Research (CCTR), Institute for Med. Laboratory Diagnostics, Wuppertal, Germany

Background and Aim: The world-wide spread of antimicrobial resistance (AMR) threatens the achievements of modern medicine, while new antimicrobial agents to treat infections due to resistant pathogens, e.g. methicillin-resistant Staphylococcus aureus (MRSA) are needed, the diagnostic tools required to guide their application are equally lacking. Thus, reference materials are key for accurate detection of antimicrobial resistance in S. aureus, especially in clinical settings. Reference materials are crucial to enforce legislation and to implement and safeguard reliable analysis and detection of methicillin resistance.

Material and Methods: The bacterial candidates for reference materials in this study were methicillin-susceptible S. aureus (MSSA), MRSA and methicillin-resistant S. epidermidis (MRSE). In the first approach, we prepared bacterial inocula of the respective colony-forming units (cfu) (1000 cfu MRSA, 100 cfu MRSA, mixture of 1000 cfu MSSA/100 cfu MRSE, and 100 cfu MRSE) directly from over-night culture and afterward these were freeze-dried and stored at $4{ }^{\circ} \mathrm{C}$ until homogeneity and stability assessment were performed. In a second approach, liquid 
Amies medium (1mL of ESwab ${ }^{\mathrm{TM}}$ ) was used for the sample production with the cfu counts as stated in the first approach. The freeze-dried samples in the first approach were reconstituted in broth medium prior to plating. For both experimental approaches, aliquots of the batches were plated on blood agar and the differential reading including cfu counting were performed after over-night incubation at $37^{\circ} \mathrm{C}$. Bacterial identification was performed by use of matrix-assisted laser desorption ionization/time of flight mass spectrometry (MALDI-TOF MS) (Bruker, Bremen, Germany) and methicillin susceptibility via Phoenix ${ }^{\circledR}$ automated testing system (BD, Heidelberg, Germany).

Results: The first approach with freeze-drying of bacterial colonies of different cfu did not reveal suitable processing due to the cfu loss in high-cfu (1000) as well in low-cfu (100) samples. In the second approach with AMIES medium, the feasibility study revealed a suitable workflow for all 4 samples. The distinction and cfu counting of the species in the sample with the mixture of $1000 \mathrm{cfu}$ MSSA/100 cfu MRSE was achieved by use of liquid-based material.

Conclusion: The liquid Amies-based materials were shown to be sufficiently homogeneous, and storage at $4^{\circ} \mathrm{C}$ up to 5 weeks did not indicate remarkable cfu loss as compared to freeze-dried samples. In the second part of the project, the feasibility of liquid Amies-based samples will be analyzed by use of genomic methods, including digital PCR and will be included into the EQA panel.

\title{
Posterwalk Mikrobiologie und Mikrobiom
}

\section{DGKL-P008}

\author{
Adhesion analysis of FISH stained bacteria using novel digital image platform
}

Mandy Sowa ${ }^{1}$; Jonas Noack ${ }^{1}$; Rico Hiemann ${ }^{1}$; Juliane Schiebel ${ }^{1}$; Jörg Weinreich ${ }^{1}$; Stefan Rödiger ${ }^{1}$; Dirk Roggenbuck ${ }^{2}$; Peter Schierack ${ }^{1}$ ${ }^{1}$ Brandenburgische Technische Universität Cottbus-Senftenberg, Multiparameterdiagnostik, Senftenberg, Germany; ${ }^{2} \mathrm{GA}$ Generic Assays GmbH, Autoimmundiagnostik, Dahlewitz, Germany

Introduction: The adhesion of bacteria is a widespread phenomenon and a crucial step in infection and colonization of hosts. Bacterial adhesion to organic material like heart valves or non-organic material like prostheses causes high risk for chronic infections. In order to determine the presence of bacteria adhered to different materials, the determination of attached bacteria species is of high interest.

Objectives: Using automated digital image VideoScan technology, the aim was to develop a tool to recognize and count bacterial cells stained with fluorescence in situ hybridization (FISH). Thereby, infection-adhesion assays should be carried out to infect human tumour cell lines and further porcine tissue sections. We intended to apply different algorithms enabling the determination of cells within diverse sizes of bacterial colonies. Material \& Methods: Using specific mathematical tools, we developed a novel algorithm for the detailed analysis of bacterial cells adherent to mammalian cells (HEp-2) and porcine tissue sections. Infections of hosts were carried out using cell line infection-adhesion assay and novel tissue infection-adhesion assay technique. Specific eubacterial FISH probe (EUB338 Atto647N) and species specific E.coli probe (ECO453 Atto647N) were used to stain bacteria. DAPI staining was done for host analysis.

Results: Using infection-adhesion assays for cell lines and tissue sections we were able to infect hosts with EPEC and EAggEC. Thereby, novel software algorithm was used to count FISH stained bacteria even in colonies. Compared to standardized technologies like lysis assay or propidium iodide staining, the FISH analysis showed higher sensitivity for the determination of bacterial cells.

Conclusion: Automated digital image analysis of FISH stained bacteria is a promising alternative for time-consuming manual methods.

\section{Posterwalk Mikrobiologie und Mikrobiom}

\section{DGKL-P009}

\author{
Rapid phenotypic distinction between methicillin-susceptible and resistant Staphylococcus aureus by use of \\ bioluminescence-based ATP assay
}

Carla Freyth'; ${ }^{1}$ Parviz Ahmad-Nejad ${ }^{1}$; Beniam Ghebremedhin ${ }^{1}$

${ }^{1}$ Helios University Clinic Wuppertal - Witten/Herdecke Univ., Centre for Clinical and Translational Research (CCTR), Institute for Med. Laboratory Diagnostics, Wuppertal, Germany

Background and Aim: Staphylococcus aureus causes a variety of suppurative and bloodstream infections. In addition, methicillin-resistant S. aureus (MRSA) is a growing threat worldwide. Early identification of MRSA involved in infection and initiation of adequate infection control measures are essential to limit MRSA spread. Moreover, the treatment of S. aureus bacteremia is challenging. Hence, rapid distinction between the methicillin-susceptible S. aureus (MSSA) and MRSA is crucial for selecting the appropriate therapy regimen since infections with MRSA are associated with increased mortality. Therefore, we developed a bioluminescence-based assay that enables to distinguish phenotypically MSSA from MRSA.

Material and Methods: The assay is based on a measurement of the bacterial concentration in a nutrient solution using bioluminescence to determine the ATP concentration produced by the bacteria. The assay was evaluated with S. aureus isolates from solid-culture medium 
$(\mathrm{n}=298)$ as well as directly from positive blood culture vials $(\mathrm{n}=73)$. Disc diffusion method, BD PhoenixTM automated susceptibility testing (Becton Dickinson, Heidelberg, Germany) and epsilometer test (Etest) were used as reference methods.

Results: This experimental distinction determines an unambiguous discrimination between methicillin-susceptible S. aureus (MSSA) and MRSA isolates. The sensitivity of the bioluminescence-based ATP assay was $97 \%$ for the analysis of solid-culture and blood culture S. aureus isolates whereas the specificity of the assay for culture media was $98 \%$. The accuracy of this method was comparable to the reference methods. Conclusion: Commonly used methods for MRSA detection, such as the disk diffusion oxacillin agar screen test or broth microdilution, automated techniques and the chromogenic ID system take normally up to 24 hours until results are available. However, the bioluminescencebased ATP assay provides an easy-to-use, cost-effective and robust detection tool for methicillin resistance in 3.5 hours and is useful at clinical sites at which rapid discrimination of MRSA from MSSA is required. Future comprehensive studies should address the time-to-effectivetherapy by use of the ATP assay.

\title{
Posterwalk Metobolom/Lipidom/Proteom/Glykom
}

\section{DGKL-P010 / PV02}

\section{Getting into depth: Integration of MS and NMR-based profiling with respect to thyroid function parameters}

\author{
Thomas Lange ${ }^{1}$; Kathrin Budde ${ }^{1}$; Georg Homuth²; Gabi Kastenmüller ${ }^{3}$; Anna Artati ${ }^{4}$; Jan Krumsiek ${ }^{5}$; Henry Völzke ${ }^{6}$; Jerzy Adamski ${ }^{4}$; Astrid
} Petersmann ${ }^{1}$; Uwe Völker ${ }^{2}$; Matthias Nauck ${ }^{1}$; Nele Friedrich ${ }^{1}$; Maik Pietzner ${ }^{1}$

${ }^{1}$ Universitätsmedizin Greifswald, Institut für Klinische Chemie und Laboratoriumsmedizin, Greifswald, Germany; ${ }^{2}$ Universitätsmedizin Greifswald,

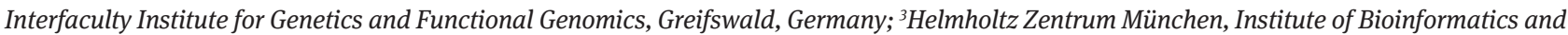
Systems Biology, Neuherberg, Germany; ${ }^{4}$ Helmholtz Zentrum München, Institute of Experimental Genetics, Neuherberg, Germany; ${ }^{5}$ Helmholtz Zentrum München, ICB (Institute of Computational Biology), Neuherberg, Germany; ${ }^{6}$ Universitätsmedizin Greifswald, Institute for Community Medicine, Greifswald, Germany

Objective: Thyroid hormones are essential regulators of human metabolism. However, a systematic investigation of associated metabolic signatures in great detail is still lacking. To this end we set up a study integrating several state-of-the-art metabolomics approaches in an epidemiological setting to associate it with the most important surrogate markers of thyroid function, namely thyrotropin (TSH), free thyroxine (FT4) and free triiodothyronine (FT3).

Methods: Plasma and urine samples from 952 participants of the Study of Health in Pomerania were analysed using proton nuclear magnetic resonance spectroscopy (1H-NMR) and mass spectrometry (MS). Hormones were measured using immunoassays. Significant hormone - metabolite associations were identified using linear regression analyses while controlling for common confounders and incorporating a possible modifying effect of sex. Sexual dimorphic associations were further followed up using a random forest-based feature selection approach to identify possible causes.

Results and Conclusion: In total, FT4 was associated with 106 out of 613 plasma metabolites. Associations with urine metabolites were far less pronounced (12 out of 587). The plasma signature was highly enriched in lipid species (e.g., free fatty acids associated positively) and in particular inverse associations with phospholipids (measured by MS) nicely aligned with a consistent decrease of large LDL- as well as small HDL-particles (measured by 1H-NMR) which could be shown for the first time. The latter was unique to men whereas associations with several polyunsaturated and saturated fatty acids were seem in women only. The feature selection approach highlighted higher alcohol consumption in men and an adverse thyroid state as well as the menopause in women as putative mediating factors. Despite T3 being the metabolic active hormone, associations with FT3 were less apparent (55 plasma and 13 metabolites in urine) partially mirroring those observed with FT4 but strikingly inverting some association directions. No associations with TSH became apparent. In general, our observations confirmed the lipolytic and lipogenic effect of thyroid hormones even in the physiological range and revealed different phenotypic characteristics, e.g. lifestyle differences, as possible confounders for sex-specific findings.

\section{DGKL-P011}

\section{Mass spectrometry based analytical quality assessment of serum and plasma specimens}

Peter Findeisen ${ }^{1}$; Shruti Hemanna ${ }^{1}$; Romi Singh Maharjan ${ }^{1}$; Victor Costina ${ }^{1}$; Ralf Hofheinz ${ }^{2}$; Michael Neumaier ${ }^{1}$

${ }^{1}$ Universitätsmedizin Mannheim, Institut für Klinische Chemie, Mannheim, Germany; ${ }^{2}$ Universitätsmedizin Mannheim, Day Treatment Center (TTZ), Interdisciplinary Tumor Center Mannheim (ITM) \& III Medical Clinic, Mannheim

Introduction: In clinical research the pre-analytical phase is the most critical part of the total analytical process and inappropriate sample handling is a major thread for any biomarker discovery approach. Up to now, most quality assurance steps are related to standard operating procedures (SOPs) and the documentation of critical steps ranging from sample collection to processing and storage. The aim of this study was to identify quality control markers (QCMs) for direct assessment of sample quality (DASQ) of human liquid samples. 
Materials and Methods: Serum and plasma specimens of healthy volunteers and patients were spiked with two synthetic reporter peptides (RPs) and aged under controlled conditions for up to 24 hours. The proteolytic fragments were monitored at various time points (1h, 4h, $8 \mathrm{~h}, 24 \mathrm{~h}$ ) by mass spectrometry (MALDI-TOF MS). The time dependent decay pattern of endogenous and exogenous peptides was used for supervised classification of samples according to their respective preanalytical quality and accuracy was determined by an independent cross validation. Results: Serum and plasma specimens have a genuine proteolytic activity that leads to a time dependent decay of peptidic QCMs. For serum and plasma six and one endogenous peptidic QCMs could be identified respectively. These were complemented with exogenous QCMs ( $\mathrm{n}=10$ and 11 for serum and plasma) that were derived from two synthetic reporter peptides. The classification accuracy for fresh specimens (1h) was $96 \%$ and $99 \%$ for serum and plasma specimens respectively, when endo- and exogenous QCMs were used for the calculations. However, classification of older specimens was more difficult and overall classification accuracy decreased to $79 \%$. Furthermore, the use of endogenous QCMs alone was clearly inferior when compared to the combination of endo- and exogenous QCMs.

Conclusion: MALDI-TOF MS is a simple and robust method that can be used for DASQ of serum and plasma specimens in a high throughput manner. We propose DASQ as a fast and simple step that can be included in multicentric large-scale projects to ensure the homogeneity of sample quality.

\section{DGKL-P012}

\section{NT-proBNP beyond cardiovascular dysfunction: metabolomic profiling implicates adiponectin as mediator of a favorable lipoprotein profile associated with NT-proBNP}

Annette Masuch ${ }^{1}$; Maik Pietzner'; Martin Bahls²; Kathrin Budde ${ }^{1}$; Gabi Kastenmüller ${ }^{3}$; Stephanie Zylla ${ }^{1}$; Anna Artati ${ }^{4}$; Jerzy Adamski ${ }^{4}$; Henry Völzke ; Marcus Dörr²; Stephan Felix²; Matthias Nauck'; Nele Friedrich ${ }^{1}$

${ }^{1}$ Universitätsmedizin Greifswald, Institut für Klinische Chemie und Laboratoriumsmedizin, Greifswald, Germany; ${ }^{2}$ Universitätsmedizin Greifswald, Innere Medizin B, Greifswald, 'Germany; ${ }^{3}$ Helmholtz Zentrum München, Institut für Bioinformatik und Systembiologie, Neuherberg,Germany; ${ }^{4}$ Helmholtz Zentrum München, Institut für experimentelle Genetik, Neuherberg,Germany; ${ }^{5}$ Universitätsmedizin Greifswald, Institut für Community Medicine, Greifswald,Germany

BACKGROUND: The N-terminal fragment of the prohormone of brain natriuretic peptide (NT-proBNP) is an important biomarker for the diagnosis of heart failure. This biologically inactive fragment is a valuable proxy for BNP as both molecules are released in equimolar concentrations from the heart. BNP is a key player in cardiovascular homeostasis inducing vasodilation and diuresis/natriuresis, and is also implicated in adipose tissue function and development. Within the pathologic setting of HF (NT-pro)BNP is typically elevated in response to increased intracardiac pressure and/or reduced cardiac output reflecting the attempt of the body to restore homeostasis.

OBJECTIVE: To explore a putative role of NT-proBNP beyond the characterization of cardiovascular dysfunction an untargeted metabolomics approach was used to investigate metabolites in plasma and urine associated with NT-proBNP measurements.

METHODS: For 872 subjects without self-reported diabetes from the population-based Study of Health in Pomerania (SHIP)-TREND with available NT-proBNP measurements comprehensive metabolomics data for plasma and urine samples were obtained by means of $1 \mathrm{H}-\mathrm{NMR}$ spectroscopy and mass spectrometry. Linear regression models were performed to assess the associations between serum concentrations of NT-proBNP and the metabolites/lipoprotein particles measured in plasma or urine. The independent cohort SHIP-2 was used as replication cohort with 1420 subjects. RESULTS: Serum concentrations of NT-proBNP showed solely significant inverse associations with seven plasma metabolites. A total of 39 urine metabolites showed significant associations with NT-proBNP. One signature comprised amino acid species including C-mannosyltryptophan and branched-chain amino acids. Inverse associations were observed for urinary levels of i.a. 11-ketoetiocholanolone glucuronide and 1-methylxanthine, while positive associations with serum NT-proBNP comprised neopterin, pseudouridine, adenosine, phosphate and others. An increase in serum NT-proBNP was associated with a benefical lipoprotein profile, including a decrease in VLDL, IDL and LDL-particles along with an increase in large HDL particles. These findings were replicated in a second independent cohort (SHIP-2). Mediation analyses revealed adiponectin as mediating factor for the association of NT-proBNP with the favorable lipoprotein profile.

CONCLUSION: As HF patients were almost absent in the present study population, the majority of the associations seen with NT-proBNP may be interpreted as attributable to the vasodilatory and the diuretic action of BNP signaling. Notably, our data suggest elevated NT-proBNP concentrations being more favorable in terms of lipoprotein status in mainly cardiac healthy individuals and hence might even exert indirect cardio-protective effects through adiponectin signaling.

\section{DGKL-P013}

\section{Targeted Metabolomics Identify Elevated Serotonin Levels in Carriers of a TCF7L2 Diabetes-Risk Allele}

Andreas Leiherer'; Axel Muendlein ${ }^{2}$; Christoph H. Saely ${ }^{3}$; Peter Fraunberger'; Heinz Drexel ${ }^{2}$

${ }^{1}$ MZL, Medizinisches Zentrallabor Feldkirch, Feldkirch, Austria;

${ }^{2}$ VIVIT, (Vorarlberg Institute for Vascular Investigation and Treatment), Dornbirn,Austria; ${ }^{3}$ LKH Feldkirch, Department of Medicine and Cardiology,, Feldkirch, Austria 
Background: The Transcription Factor 7-like 2 (TCF7-L2) is part of the Wnt signaling pathway. Its polymorphism rs7903146 is known to be associated with a risk for metabolic diseases, primarily diabetes mellitus, whereas the molecular mechanisms explaining how TCF7L2 impacts metabolism have remained unsolved.

Methods: We evaluated the metabolic profile of a total of 394 angiopraphied patients with respect to their rs7903146 genotype (C/T) using targeted metabolomics in a discovery $(n=154)$ and a validation $(n=240)$ study.

Results: We have identified serotonin as the top metabolite being increased in carriers of the risk allele (T) in both studies. For all 394 patients fold change was $44.6 \%(\mathrm{p}=2.06 \mathrm{e}-5)$. In addition we also found an increase in glycerophospholipids. Serotonin was significantly associated with the rs7903146 genotype after full adjustment including diabetes and the further top ranked metabolites.

In a larger cohort of 1660 similar patients, we found that risky allele carriers had a significantly higher fasting glucose (116 vs. $109 \mathrm{mg} / \mathrm{dl}$, $\mathrm{p}=0.007$ ), post-challenge glucose ( $147 \mathrm{vs.} 135 \mathrm{mg} / \mathrm{dl}, \mathrm{p}=0.036)$, HbA1c (6.2 vs. $6.0 \%, \mathrm{p}<0.001)$, and prevalence of T2DM (30.8 vs. $25.8 \%$, $\mathrm{p}=0.022$ ) than patients without the risky allele. Apart from rs7903146, analyzing the whole gene, we found that 9 out of 19 unlinked SNPs in TCF7L2 were significantly associated with serotonin as well.

Conclusion: In conclusion, this study identifies a significant association between elevated serotonin concentrations and the diabetes risk allele of a TCF7L2 polymorphism. Recently, Wnt-signalling has been suggested to be involved in serotonin expression and serotonin has been shown (i) to regulate glucose homeostasis, (ii) to increase the risk of diabetes, and (iii) to be elevated in diabetic subjects. Together, these new findings suggest that serotonin may be, at least in part, involved in the impact of Wnt/TCF7L2-signalling on metabolic homeostasis and diabetes.

\title{
Posterwalk Metobolom/Lipidom/Proteom/Glykom
}

\section{DGKL-P014}

\section{Postprandial plasma choline metabolites respond differently after glucose and fat tolerance tests in healthy subjects}

Juergen Geisel'; Astrid Knell'; Hussain Awwad'; Ulrich Hübner'; ${ }^{1}$ Rima Obeid ${ }^{1}$

${ }^{1}$ Universitätsklinikum des Saarlandes, Klinische Chemie, Homburg, Germany

Background: Plasma choline shows associations with plasma glucose and lipids. We studied changes of choline metabolites after oral glucose tolerance test (OGTT) and after oral fat tolerance test (OFTT). Methods: Healthy subjects ( $\mathrm{n}=18$, mean age $=54.3 \mathrm{yrs}$; BMI $=26.8$ $\mathrm{kg} / \mathrm{m} 2$ ) underwent 2 tests within 1 week. First, OFTT (= $250 \mathrm{~g}$ cream drink containing $80 \mathrm{~g}$ fat) was applied and blood samples were collected prior to and 4 hours after OFTT. Seven days later, $75 \mathrm{~g}$ glucose was applied and blood was collected prior to and 2 hours after OGTT. Plasma concentrations of choline, betaine, trimethylamine N-oxide (TMAO), dimethylglycine, S-adenosylmethionine (SAM), lipids and glucose were measured. Results: After OFTT, plasma choline declined (mean from 9.2 to $10.6 \mu \mathrm{mol} / \mathrm{L} ; \mathrm{p}=0.004$ ), betaine declined (33.4 to $31.7 \mu$ mol/L; $\mathrm{p}=0.003$ ), TMAO tended to increase ( 4.1 to $5.6 \mu \mathrm{mol} / \mathrm{L} ; \mathrm{p}=0.105$ ), glucose declined ( 5.39 to $4.98 \mathrm{mmol} / \mathrm{L} ; \mathrm{p}<0.001$ ), and triglycerides increased (1.27 to $2.53 \mathrm{mmol} / \mathrm{L} ; \mathrm{p}<0.001)$. All other markers remained unchanged after OFTT. After OGTT, plasma choline increased (10.1 to 11.1 $\mu \mathrm{mol} / \mathrm{L} ; \mathrm{p}<0.001$ ), TMAO declined ( 4.0 to $3.5 \mu \mathrm{mol} / \mathrm{L} ; \mathrm{p}=0.029)$, dimethylglycine declined (2.0 to $1.7 \mu \mathrm{mol} / \mathrm{L} ; \mathrm{p}=0.005)$, SAM declined (103 to $96 \mathrm{nmol} / \mathrm{L} ; \mathrm{p}=0.041$ ), but betaine, glucose, and SAM remained unchanged. Concentrations of all metabolites measured before applying the 2 tests did not differ within 1 week. Conclusion: OGTT reduced the production of methyl groups and lowered plasma TMAO in healthy individuals. Whereas, OFTT caused a diverse response of plasma TMAO that is not explained by changes in other choline metabolites.

\section{DGKL-P015}

\section{Simple and sensitive detection of TTR-mutations in serum specimens by liquid chromatography / mass spectrometry (LC-MS/MS)}

\author{
Peter Findeisen ${ }^{1}$; Victor Costina ${ }^{1}$; Michael Neumaier ${ }^{1}$ \\ ${ }^{1}$ Universitätsmedizin Mannheim, Institut für Klinische Chemie, Mannheim, Germany
}

Introduction: Transthyretin (TTR) is a low molecular weight (127 aa) medium abundant plasma protein of homotetrameric structure. It is involved in thyroid hormone transport as well as in retinol binding. Point mutations in this protein are known to be associated with the formation of amyloid deposits causing e.g. heart failure or neurological disorders. This TTR amyloidosis is an autosomic dominant disease and phenotypic manifestation is mainly related associated to the location of mutations and respective aminoacid-substitutions. Due to the variability of symptoms, the time to diagnosis might take up to several years. However, therapeutic options are available and the prognosis of TTR amyloidosis can be improved by their timely initiation. Accordingly, a sensitive and specific screening assay is desirable for this rare disease. Materials and Methods: We have developed a LC-MS/MS (liquid chromatography / tandem mass spectrometry) based method that has been validated on more than 100 samples. Immunoprecipitation of TTR was performed prior to proteolytic digestion with trypsin and Arg-C. Patients were diagnosed by molecular sequencing methods in parallel. 
Results: The workflow showed very high protein sequencing coverage of up to $100 \%$. Mutations such as Val30Met, Ile107Val, Glu54Gln and Gly6Ser were securely detected. The comparison of results with nucleic acid sequencing showed concordance in any case.

Conclusion: Mass spectrometry based protein sequencing has evolved as new technology that offers high resolution, adequate throughput and low cost for sensitive identification of TTR mutations in minimum amount of serum specimens.

\section{Posterwalk Molekulare Diagnostik}

\section{DGKL-P016 /PV03}

\section{Cell-free DNA as non-invasive biomarker of cardiac allograft rejection}

Franziska Knüttgen ${ }^{1}$; Julia Beck²; Lena Reinhard³ ${ }^{3}$ Armin Zittermann ${ }^{4}$; Michael Oellerich ${ }^{3}$; Uwe Schulz $;$; Uwe Fuchs ${ }^{4}$; Cornelius Knabbe ${ }^{1}$; Tobias Flieder ${ }^{1}$; Katharina von Bargen ${ }^{1}$; Ekkehard Schütz ${ }^{2}$; Jan Gummert ${ }^{4}$; Ingvild Birschmann ${ }^{1}$

${ }^{1}$ Herz- und Diabeteszentrum Nordrhein-Westfalen, Universitätsklinik der Ruhr-Universität Bochum, Institut für Laboratoriums- und Transfusionsmedizin, Bad Oeynhausen, Germany; ${ }^{2}$ Chronix biomedical, Chronix biomedical, Göttingen, Germany; ${ }^{3}$ Dept. Clinical Pharmacology University Medical Center, Dept. Clinical Pharmacology University Medical Center, Göttingen, Germany; ${ }^{4} H e r z-$ und Diabeteszentrum NRW, Universitätsklinik der Ruhr-Universität Bochum, Klinik für Thorax- und Kardiovaskularchirurgie, Bad Oeynhausen, Germany

Objective: Currently, the only valid method for the diagnosis of allograft rejection after heart transplantation (HTx) is the invasive myocardial biopsy. Since this diagnostic is still associated with a number of problems, the search for a valid non-invasive biomarker is of central interest. We investigated the potential of circulating graft-derived cell free DNA (GcfDNA) as such a non-invasive biomarker in a cohort of cardiac transplant recipients.

Methods: Plasma samples from a total of 87 cardiac transplant recipients were analyzed by a droplet digital PCR method in order to determine the GcfDNA percentages. A previously defined set of over 40 single nucleotide polymorphisms was used to distinguish donor and recipient cfDNA. From this set 4 - 5 informative assays per donor-recipient pair were selected to quantify GcfDNA as previously described (Beck et al, CLin Chem 2013). The determined GcfDNA values were then correlated with the occurrence of rejections. The diagnosis of rejections was based on clinical parameters and a biopsy-based grading system.

Results: The average course of GcfDNA for the first postoperative year was presented from a total of 780 samples. It became apparent that the values of GcfDNA in the first hours and days after HTx due to ischemia/reperfusion damage have been significantly increased. Within the first 10 postoperative days, the median of the GcfDNA decreases below the cutoff of $0.345 \%$. Samples obtained after the first 10 days after HTx could be used to assess the association between GcfDNA values and cardiac rejection. For this purpose, the samples were divided into groups according to defined criteria: 'stable phase', 'ISHLT OR', 'ISHLT 1R', 'ISHLT 2R', 'clinical suspicion' and samples collected ' 9 - 30 days before rejection'. Samples in groups 'ISHLT 1R' and 'ISHLT 2R' show significantly higher GcfDNA values than samples of 'stable phase' (P < 0.001-0.0001). Highest GcfDNA values were obtained in samples collected during biopsy proven rejection grade $2 \mathrm{R}$. The samples of the groups 'clinical suspicion' and ' 9 - 30 days before rejection' also show significantly higher values than the 'stable phase' $(\mathrm{P}<0.0001)$. The influence of invasive biopsies on the GcfDNA (samples taken within 12 hours after biopsy) was also investigated. It was found that only biopsy sampling, regardless of the result of the biopsy, caused a significant increase $(\mathrm{P}<0.0001)$ in the proportion of GcfDNA compared to the samples of the 'stable phase'. Conclusion: To further improve the sensitivity and specificity of the method, it will be necessary to examine the various effects of the preanalytics more closely. Nevertheless, GcfDNA values correlate highly significant with cardiac rejection. In addition, samples collected ' 9 30 days before biopsy proven rejection' show significantly higher GcfDNA values than those of the 'stable phase'. This indicates the potential of GcfDNA as a non-invasive early rejection and graft damage marker in HTx.

\section{DGKL-P017}

\section{A simple and non-invasive assay for Characterization of aberrant mRNA-Splicing by NMD inhibition}

Friederike Häuser'; Gesa Werner'; ${ }^{1}$ Karl Lackner ${ }^{1}$; Heidi Rossmann ${ }^{1}$

${ }^{1}$ Universitätsmedizin Mainz, Institut für Klinische Chemie und Laboratoriumsmedizin, Mainz, Germany

Background: The precise identification and characterization of genetic variants in monogenic diseases has a wide influence on diagnosis and therapy. About $10 \%$ of pathogenic variants are splicing variants. Due to the complex mechanism of splice regulation it is difficult to predict the effects of variants on mRNA splicing. Possible consequences are exon skipping, intron retention, generation of novel splice sites, or the utilization of a cryptic splice site. Common consequences are a frame-shift and the generation of premature termination codon. This leads to RNA degradation via the nonsense mediated decay (NMD) pathway. Usually NMD testing is performed in patient's skin fibroblasts which are obtained by skin biopsy. We developed an approach in order to characterize novel splicing mutations in a simple and non-invasive manner, which is also applicable to patients of other medical centres. 
Material and method: Blood samples (NH4 + heparin) from healthy probands were stored at different conditions for up to one week in order to test the viability of the blood cells and their suitability for downstream assays after a long-distance sample transport. Isolated blood lymphocytes from healthy probands were cultured in standard leucocyte medium supplemented with different concentrations of NMD inhibitors for 6,16 and $24 \mathrm{~h}$. We used NMD inhibitors for in vitro use only (inhibition of translation) as well as potential drugs for a therapeutic intervention. The cells were harvested and RNA was isolated. Success of NMD inhibition was quantified by qPCR.

Results: Transport or storage of blood samples for subsequent NMD assay is possible for up to three days. Compared to the non-stimulated lymphocyte controls, nonsense mediated RNA decay is inhibited in an optimal way by anisomycin stimulation for $6 \mathrm{~h}$. However, the incubation of lymphocytes with further agents (as non-translation inhibiting NMD inhibitors) is also successful. The assay has already been applied in order to characterize splice site mutations in patients with lysosomal storage disease.

Discussion: RNA analysis in lymphocytes with and without NMD inhibition is a simple method for analysing splice defects in all monogenic disorders with expression of the disease causing gene in lymphocytes. A further advantage for the patient is the use of blood cells instead of fibroblasts, because a skin biopsy can be avoided and analysis times are reduced. Successful inhibition of NMD in vitro might help to predict which patients can profit from a therapeutic intervention with NMD inhibitors. Even expression of a partial protein with low or no activity reduces the risk for the patient to develop antibodies interfering with enzyme/protein replacement therapy.

\section{DGKL-P018}

\section{First insights into the senescence associated secretory phenotype and its possible role in Pseudoxanthoma elasticum}

Janina Tiemann ${ }^{1}$; Thomas Wagner'; ${ }^{1}$ Thanh-Diep Ly ${ }^{1}$; Bettina Ibold ${ }^{1}$; Olivier Vanakker ${ }^{2}$;sabel Faust ${ }^{1}$; Cornelius Knabbe ${ }^{1}$; Doris Hendig ${ }^{1}$

${ }^{1}$ Herz- und Diabeteszentrum NRW, Universitätsklinik der Ruhr-Universität Bochum, Institut für Laboratoriums- und Transfusionsmedizin, 32545 Bad Oeynhausen, Germany, Institut für Laboratoriums- und Transfusionsmedizin, Bad Oeynhausen, Germany; ${ }^{2}$ Center for Medical Genetics, Ghent University Hospital, 9000 Ghent, Belgium, Center for Medical Genetics, Ghent, Belgium

Background: Aging is defined as a progressive decline of physiological function and integrity. The molecular processes underlying it are very heterogenic and not fully understood. Studies on rare progeria syndromes, which display an accelerated progression of physiological aging (e.g. Hutchinson-Gilford-progeria syndrome), can help to get a better understanding. Pseudoxanthoma elasticum (PXE) which is mainly caused by mutations in the ATP-binding cassette sub-family C member 6 (ABCC6) gene shares some molecular characteristics with such premature aging diseases. A better insight into those processes could be beneficial for the understanding of PXE pathogenesis as well as for the progression of healthy physiological aging. Thus, the aim of this study was to examine aging biomarkers in sera and dermal fibroblasts of PXE patients and appropriate healthy controls.

Methods: Normal human dermal fibroblasts (NHDF) $(n=3)$ and fibroblast from PXE patients $(n=3)$ were seeded with a density of 177 cells/ $\mathrm{mm}^{2}$. The medium was changed after $24 \mathrm{~h}$. The measurement of the ß-galactosidase activity as a marker for cellular senescence was conducted $72 \mathrm{~h}$ after initial medium change. Activity was evaluated by measurement of fluorescence. Evaluation of mRNA expression was performed by quantitative polymerase chain reaction 21d after initial medium change. During the 21d of cultivation, medium was refreshed every 3 to 4 days. We measured the mRNA expression of growth differentiation factor 11 (GDF11) and interleukin 6 (IL6) as marker for aging and a senescence associated secretory phenotype (SASP). GDF11, eotaxin and IL6 were quantified by immunoassays in cell culture supernatants and serum from PXE patients $(n=45)$ and age/sex-adjusted healthy controls $(n=45)$.

Results: We examined a significant increase in ß-galactosidase activity of fibroblasts from PXE patients compared to NHDF. Furthermore, we observed a 9-fold increase in IL6 and a 0.3-fold decrease of GDF11 mRNA expression in fibroblasts from PXE patients. Preliminary data showed increased IL6 protein concentrations in supernatants of PXE fibroblasts. GDF11 plasma levels were decreased in older healthy controls ( $>45$ years) compared to young healthy controls ( $<45$ years) as well as in PXE patients compared to the appropriate healthy controls although the difference did not reach statistical significance. Plasma levels of eotaxin showed a 1.5-fold increase with age in PXE patients as well as in healthy controls. However, no statistical different concentrations were detected between PXE patients and healthy controls.

Conclusion: Our data indicate that PXE pathogenesis goes along with molecular characteristics of premature aging. In this context, potential chronic inflammatory processes due to an increase of senescent cells and the development of a SASP in ABCC6 deficient dermal fibroblasts may contribute to the progression of the disease.

\section{DGKL-P019}

\section{A compound heterozygous XYLT1 deletion in dermal fibroblasts of a patient with short limb skeletal dysplasia leads to abnormal myofibroblast characteristics}

Bastian Fischer ${ }^{1}$; Thanh-Diep Ly ${ }^{1}$; Joachim Kuhn ${ }^{1}$; Doris Hendig ${ }^{1}$; Cornelius Knabbe ${ }^{1}$; Isabel Faust ${ }^{1}$

${ }^{1}$ Herz- und Diabeteszentrum Nordrhein-Westfalen, ILTM, Bad Oeynhausen, Germany

Background: Xylosyltransferases (XT), of which two human isoforms exist, catalyze the rate-limiting step during the proteoglycan (PG) biosynthesis. PG are major components of the extracellular matrix (ECM) and play a key role in different processes, as for instance development. 
Fibroblasts play an essential role with regard to the remodelling of the ECM. The cytokine transforming growth factor beta 1 (TGF- $\beta 1$ ) induces the differentiation of fibroblasts into myofibroblasts, which have an impact on the wound healing process and the remodelling of the ECM as well. Deletion mutations in XYLT1 are associated with different diseases, as for example Desbuquois skeletal dysplasia (DBSD). In contrast, an elevated XT-activity leads to an accumulation of PG within the ECM and thus to fibrosis. Consequently, an enhanced myofibroblast differentiation takes place. In our study, we analysed fibroblasts of a young patient with neonatal short limb skeletal dysplasia, which is a special type of DBSD. A genetic profiling previously revealed a compound heterozygous XYLT1 deletion in this patient. We were interested in the question, if such a deletion leads to differences in myofibroblast differentiation and mRNA expression of ECM-associated targets.Methods: Dermal fibroblasts of healthy controls $(n=2)$ and dermal fibroblasts of the patient were harvested, total RNA was isolated and gene expression levels of different ECM-associated targets were determined by quantitative realtime-PCR. For the wound healing assay, an artificial gap was created on a confluent cell monolayer. Migration of the fibroblasts towards this scratch was observed hourly by live cell monitoring. To compare the contractility of control and patient fibroblasts, cells were seeded on a collagen type 1 gel. After adhesion, cells were treated with TGF- $\beta 1$ and the gel was photographed at different points in time. To determine the contractility, the gel area was quantified using ImageJ. The XT activity in cell culture supernatants was measured in a radiochemical assay. Results: Compared to controls, determined gene expression levels of the ECM-associated targets XYLT1, ACAN, COL1A1 and ACTA2 were all highly significant decreased in patient-fibroblasts. As opposed to this, the TGF- $\beta 1$ expression level was elevated in the patient-fibroblasts. Extracellular XT-activity was highly significant diminished in the supernatant of the patient cells. Patient fibroblasts showed a decreased contractility compared to control cells after TGF- $\beta 1$ induction, but no significant differences in wound healing properties.Conclusion: Due to possible pathological changes, fibroblasts of our patient with a compound heterozygous XYLT1 deletion show, in comparison to control fibroblasts, diminished contractility and XT-activity as well as abnormal mRNA expression levels of different ECM-associated genes. Our data show for the first time, that a compound heterozygous XYLT1 deletion results in abnormal myofibroblast characteristics.

\title{
DGKL-P020
}

\section{Validation of an extraction method for microplastics from human materials}

\author{
Adrian Monteleone; Andreas Fath; Folker Wenzel \\ Hochschule Furtwangen, Medical and Life Sciences, Villingen Schwenningen,
}

Introduction: Since the beginning of industrial production in 1950, plastic production has continued to grow strongly worldwide and is now at 322 million tonnes in the year 2015. From these very high production volumes ever larger quantities are found in the environment. There the plastics degradate to microplasticity and spread ubiquitously in the world. The present work deals with the possible uptake of microplastic particles in human organisms. For the detection of these plastic particles, an extraction method was developed and validated.

Materials and Methods: Biological materials consist of human blood (healthy volunteers, $\mathrm{n}=7$ ) and different tissues of pigs and cattles. Various lysis solutions were tested for degradation efficiency of biological material and for effects on the plastics. The mass loss, surfaces and structure variations as well as the physicochemical spectrum of the material were observed after treatment by atomic force (AFM) and electron microscopy (EM) and Fourier transform infrared spectrometry (FTIR).

Results: The different plastic types as polyamide (PA), polycarbonate (PC), polyethylene (PE), polypropylene (PP), polystyrene (PS) and polyvinyl chloride (PVC) could be clearly differentiated and identified by FTIR. Regarding the surface control, especially PVC showed detectable alterations: After extraction an irregular surface structure caused by protuberances or bubbles could be observed. However, instead of these alterations an equivalent count of plastic particles was found in correlation to the applied plastic amount (recovery rate overall was $99,49 \pm$ $0,59 \%)$.

Conclusion: The applied method can be used for plastic extractions from human or animal tissues without remarkable effects on the plastics.

\section{DGKL-P021}

\section{Molecular pathologic examinations for small scale laboratories}

\author{
Anja Meyer ${ }^{1}$; Peter Stömmer ${ }^{2}$ \\ ${ }^{1}$ Pathologie Augsburg Hermannstr., Molekularpathologie, Augsburg, Germany; ${ }^{2}$ Pathologie Augsburg Hermanstr., Pathologie, Augsburg, Germany
}

Background: The fast development of molecular biological methods in pathology offers numerous opportunities for an increase in diagnostic precision especially in the pathology of tumors and infections. But new methods like NGS also provide new problems in handling these technically sophisticated methods as well as their analysis and interpretation. To finally come to significant results of high quality, it is necessary to analyze the generated data by qualified personnel, which is often not available in each pathological lab.

Methods: Here we present two different molecular diagnostics methods which offer fast and easy access to clinical molecular diagnostic information: The fully automated, real-time PCR based system Idylla (Biocartis) and non-automated assays using conventional PCR followed 
by a strip assay for reverse hybridization (GenID). Both systems enable clinical laboratories to perform a broad range of applications in oncology, infectious diseases and beyond.

Results: Both methods produce reliable results for the detection of clinical relevant mutations from tumor samples in FFPE for the KRAS(Exons 2 codon 12/13, 3 codon 59/61, 4 codon 117/146) and BRAF-Gene (Exon 15, Codon 600-601). Furthermore we obtain significant results for the detection of HPV-DNA from vaginal brushings and FFPE samples (GenID). Finally our ability to generate significant testing was proofed by interlaboratory comparison and our lab was accredited by DAKKS for DIN EN ISO/IEC 17020:2012.

Conclusion: There are techniques available which also enable small-sized pathology labs for offering molecular pathologic examinations. Once established, these techniques can be carried out without the need for specialized personnel even in an accredited mode.

\title{
DGKL-P022
}

\section{Regulation of Xylosyltransferase I Expression by Activin A in Human Skin Fibroblasts: Signaling Pathway and Inhibition of Xylosyltransferase I Activity as new Approach for the Treatment of Dermal Fibrosis?}

Thanh-Diep Ly; Ricarda Plümers; Bastian Fischer; Doris Hendig; Joachim Kuhn; Cornelius Knabbe; Isabel Faust Institut für Laboratoriums- und Transfusionsmedizin, Herz- und Diabeteszentrum Nordrhein-Westfalen, Universitätsklink der Ruhr-Universität Bochum, Bad Oeynhausen, Germany, Institut für Laboratoriums- und Transfusionsmedizin, Bad Oeynhausen, Germany

Introduction: Skin fibrosis occurs in a variety of human diseases, emerging in terms of hypertrophic scarring or systemic sclerosis (SSc), the prototype of fibrotic skin diseases. The hallmark of this disorder is an excess deposition of collagenous fibers and extracellular matrix proteins in the dermis. The transition of fibroblasts to myofibroblasts in the presence of fibrotic mediators like interleukin-13, $-4,-6$, monocyte chemoattractant protein 1 (MCP-1) or activin A, a cytokine of the transforming growth factor-beta superfamily, reveals a key event of this process. However, the molecular mechanism by which fibrosis is induced has not been fully understood. In humans, the first step in the biosynthesis of proteoglycans is the addition of a xylose to a core protein. This initial step is catalyzed by one of two xylosyltransferases (XT), XT-I and XT-II. Human XT-I is a marker for myofibroblast differentiation and its increase in enzymatic activity refers to the abnormal rate of proteoglycan biosynthesis under fibrotic conditions.

Objectives: The aim of our study is to understand the underlying mechanism for the regulation of human XT-I. We hypothesize that one mechanism by which activin A contributes to dermal fibrosis is mediated by upregulation of XYLT1 expression in dermal fibroblasts. Therefore, we investigated the regulation of XYLT1 expression in normal human dermal fibroblasts (NHDF) by activin A and the underlying signaling pathway.

Methods: The expression of XYLT1 and associated target genes in cultured NHDF stimulated with activin A was assessed by quantitative real time PCR. The determination of intracellular and extracellular XT activity of NHDF was performed by radiochemical activity assay. The underlying signaling pathways were investigated using antibody arrays. To evaluate a potential therapeutic strategy for fibrosis, the inhibition of XT-I activity was evaluated at both enzymatic and signaling stage by addition of different inhibitors.

Results: Activin A is a potent regulator of XYLT1 expression in NHDF, whereas the treatment of NHDF with the profibrotic mediators interleukin-4, -13 or -6 and MCP-1 resulted in no significant induction of XYLT1 mRNA expression. Furthermore, we have determined the signaling pathway of XYLT1 expression in dermal fibroblasts after activin A stimulation. Our data show that XYLT1 is mainly regulated via activin receptor type IB pathway in a SMAD7-dependent manner. Furthermore, we were able to demonstrate the involvement of non-canonical mitogenactivated protein kinase pathway components in activin A-mediated XYLT1 induction.

Conclusion: Activin A increase the inflammatory and fibrotic potential of dermal fibroblasts through the upregulation of XT-I, a marker for myofibroblast differentiation in skin fibrosis and inflammatory disease. We could show the inhibition of XT-I-activity via components of the activin A signaling pathway to be a future approach for the treatment of SSc.

\section{DGKL-P023}

\section{Hepatitis E virus NAT blood donor screening - from bench side decision to mandatory national screening?}

\author{
Tanja Vollmer'; Benjamin Müller²; Jürgen Diekmann³ Matthias Eberhardt ${ }^{4}$; Cornelius Knabbe ${ }^{3}$; Jens Dreier ${ }^{3}$ \\ ${ }^{1}$ Herz- und Diabeteszentrum Nordrhein-Westfalen, Institut für Laboratoriums- und Transfusionsmedizin, Bad Oeynhausen, Germany; \\ ${ }^{2} \mathrm{Herz}$ - und Diabeteszentrum NRW, Universitätsklinik der Ruhr-Universität Bochum, Institut für Laboratoriums- und Transfusionsmedizin, \\ Bad Oeynhausen,Germany; ${ }^{3}$ Herz- und Diabeteszentrum Nordrhein-Westfalen, Universitätsklinik der Ruhr-Universität Bochum, Institut für \\ Laboratoriums- und Transfusionsmedizin, Bad Oeynhausen,Germany; ${ }^{4}$ TMD Gesellschaft für transfusionsmedizinische Dienste mbH, Kassel, \\ Germany
}

Background: The risk and importance of transfusion-transmitted hepatitis E virus (TT-HEV) infections in line with the question of a general HEV-NAT screening of blood products is currently a highly controversial discussed topic. Just recently, the German Advisory 
Committee on Blood announced the intention of a mandatory NAT screening for all therapeutic blood products starting from September 2019 (sensitivity: 2,000 IU HEV RNA/ml per single donation). Our transfusion facility was one step ahead of this issue and already introduced HEV-NAT screening for years after detection of a high frequency of viremic donors in 2011. Here, we present our experience of the last three years with the successful implementation of a 100\% screening of therapeutic blood products for HEV RNA, emphasizing the importance of this regulatory approach. Since the infectious dose remains an unknown quantity, we systematically review the currently known cases of TT-HEV infections and available routine NAT-screening assays to gain insight into the minimum required sensitivity, and possible screening approaches.

Methods: From January 2015 to December 2017, 288,724 donations from 57,498 donors were screened in minipools (MP) of 96 samples for the presence of HEV RNA using the RealStar HEV RT-PCR assay (Altona Diagnostics GmbH). Nucleic acids were extracted from $4.8 \mathrm{ml}$ plasma using the Chemagen MSM-I extractor (Viral 5k, Perkin Elmer Chemagen GmbH). The presence of HEV-specific IgM and IgG antibodies was determined using the anti-HEV IgM/IgG ELISA (Euroimmun, Luebeck). Follow-up samples were also tested for the presence of HEV RNA and HEV-specific antibodies.

Results: 199 HEV RNA positive donors were identified, corresponding to an average yearly incidence of $0.075 \%$. A total of 45 donors showed reactive IgM and/or IgG antibody titers (IgM +/IgG- (1.10\%), IgM +/IgG + (14.84\%), IgM-/IgG + (8.79\%)), the remaining 154 donors were HEV NAT-only positive. Merely $23.07 \%$ of HEV RNA positive donors had elevated ALT levels. Follow-up samples are currently available for 153 donors. In all of these individuals, an IgG seroconversion was observed. In the case review analysis, all blood components with an infectious dose (IFD) above 5.00E + 04 IU (definite IFD) caused an infection. The lowest IFD resulting in TT-HEV infection observed was 7.05E + 03 IU (minimal IFD). Based on these results and the amount of plasma in different blood products, we extrapolated the detection probability of HEV-RNA positive blood donors using different test strategies (NAT assay, ID vs. MP-NAT with different pool sizes).

Conclusions: The high number of identified HEV RNA positive donors emphasizes the need for HEV-NAT screening to increase the safety of blood products. MP-NAT screening (up to 96 using assays with 95\% LOD of $20 \mathrm{IU} / \mathrm{ml}$ ) of blood donations seems to be suitable as a routine screening assay to identify donors carrying viral loads in the range of the definite IFD and to cover a large part of viremic phases.

\section{DGKL-P024}

\section{microRNA-29b mediates fibrotic induction of human xylosyltransferase-I}

Lara Riedel; Bastian Fischer; Thanh-Diep Ly; Doris Hendig; Joachim Kuhn; Cornelius Knabbe; Isabel Faust

Herz- und Diabeteszentrum Nordrhein-Westfalen, Institut für Laboratoriums- und Transfusionsmedizin, Bad Oeynhausen,Germany

Background: Human xylosyltransferase-I (XT-I) is a golgi-resident enzyme, which catalyzes the rate-limiting step in proteoglycan glycosylation and is secreted in the extracellular space. Quantification of human XT-I activity in human serum or tissue reflects proteoglycan biosynthesis rate and therefore represents a non-invasive fibrosis biomarker. Furthermore, human XT-I is a cellular mediator of myofibroblast differentiation - the key step of fibrotic tissue remodeling, which is defined by accumulation of extracellular matrix molecules as for instance proteoglycans. A therapeutic approach for fibrotic diseases has not been established yet. Our current studies deal with the question, whether the interference with XT-I or associated regulatory pathways might be a suitable anti-fibrotic approach. The aim of this study was to find out whether XT-I might be regulated by microRNA29b, a key player in fibrosis.

Methods: microRNA target prediction was performed using online in silico tools. Binding of microRNA-29b to the XYLT1 3’UTR was examined by transfecting SW1353 cells with microRNA-29b and an appropriate XYLT1 3`UTR coding plasmid, followed by quantification of luciferase activity. To analyze the influence of microRNA-29b or its inhibitor on mRNA expression of XYLT1 and associated genes, normal human dermal fibroblasts (NHDF) were transfected. Gene expression analyses and quantification of XT activity were realized by quantitative realtime PCR and a radiochemical assay, respectively. To quantify specificity protein 1 (SP1) protein amount, an ELISA was performed. Identification of SP1 binding sites in the XYLT1 promoter region was conducted by in silico analyses, while SP1 binding sites in the promoter were deleted by site-directed mutagenesis. The influence of SP1 binding site deletion on XYLT1 promoter activity was monitored by a dual luciferase assay and SP1/SP3 binding to XYLT1 promoter region was verified via an electrophoretic mobility shift assay (EMSA).

Results: While we could demonstrate that XYLT1 is neither an in silico identified direct target of microRNA-29b nor an experimental confirmed 3'UTR binding partner of microRNA-29b, transfection of NHDF with microRNA-29b or its inhibitor affected both XYLT1 mRNA expression and XT activity. Combined results of the target prediction analysis and transfection experiments pointed out that there might be an indirect XT-I regulation via the transcription factor SP1. We identified seven SP1 binding sites in the XYLT1 promoter region and could subsequently show that their elimination decreased XYLT1 promoter activity. Occupancy of all predicted SP1 promoter binding sites was finally verified by an EMSA.

Conclusions: Based on our results we are able to suggest a hitherto not described microRNA-29b-mediated regulation pathway of human XT-I via SP1. Thus, the connection of these two important fibrotic players, microRNA-29b and XT-I, will improve our understanding of the complex fibrotic molecular pathways. 


\title{
Posterwalk Endokrinologie, Stoffwechsel und Herz/Kreislauf I
}

\section{DGKL-P025 / PV04}

\author{
The role of Formyl Peptide Receptor 1 in the development of non-alcoholic steatohepatitis
}

Marina Nati'; Antonios Chatzigeorgiou ${ }^{1}$; Lan-Sun Chen ${ }^{1}$; Ales Neuwirth ${ }^{1}$; Jochen Hampe ${ }^{2}$; Oliver Soehnlein ${ }^{3}$; Kyoung-Jin Chung ${ }^{1}$; Triantafyllos Chavakis $^{1}$

${ }^{1}$ Institute for Clinical Chemistry and Laboratory Medicine, Faculty of Medicine Technische Universität Dresden, Dresden, Germany; ${ }^{2}$ Department of Medicine I, Faculty of Medicine Technische Universität Dresden, Dresden, Germany; ${ }^{3}$ Institute for Cardiovascular Prevention, LudwigMaximilians-Universität München, Munich, Germany

Non-alcoholic fatty liver disease (NAFLD) affects one in four people worldwide and, despite the enormous clinical and economic burdens, there are currently no approved pharmacological treatments for this pathology. The severe necroinflammatory form of NAFLD, non-alcoholic steatohepatitis (NASH), is one of the leading causes of liver transplantation because of its potential progression to liver cirrhosis and hepatocellular carcinoma. Recent studies have shown that, among NASH histological characteristics, only the fibrosis stage predicts this progression and the disease-specific mortality. The crucial step in hepatic fibrosis development is known to be the transdifferentiation of hepatic stellate cells (HSCs) into fibrogenic myofibroblasts, in response to liver injury; however, the exact underlying mechanisms have not been fully elucidated. Here we show that Formyl Peptide Receptor 1 (FPR1), a G protein-coupled receptor known for triggering the innate immune response by sensing necroinflammation, plays a direct role in NASH-related hepatic fibrosis.

Interestingly, we found that the hepatic expression of FPR increases during the development of NASH and positively correlates with the expression of liver fibrosis markers, both in mice and in humans. Consistently, when exposed to a diet-induced model of NASH, FPR1-deficient mice displayed blunted HSC activation and consequent hepatic fibrosis compared to FPR1-sufficient mice. Unexpectedly, FPR1 deficiency protected from liver fibrosis without affecting liver inflammation, damage, and steatosis. Moreover, by performing bone marrow chimera experiments, we could indeed demonstrate that the phenotype was attributable to the absence of FPR1 in liver resident cells, while FPR1 expression in bone marrow-derived immune cells was dispensable for fibrosis development. Consistently, we found that HSCs expose FPR1 on their surface and FPR1 expression increases upon activation in vitro and NASH-associated liver fibrosis development. Furthermore, treating HSCs with the FPR1 agonist fMLP promoted their migration, activation, and collagen deposition in vitro. Interestingly, in vivo pharmacological blockade of FPR1 protected from NASH-induced fibrosis in mice.

Taken together, we show that the interaction between formylated peptides and FPR1 promotes hepatic fibrosis in an HSC-intrinsic manner, independently of inflammation. Therefore, FPR1 might represent a novel specific target for the treatment of NASH-induced liver fibrosis.

\section{DGKL-P026 / PV05}

\section{Systems medicine approach combining Multi-omics and functional analyses in hepatic and skeletal muscle mitochondria of pre-diabetic mice}

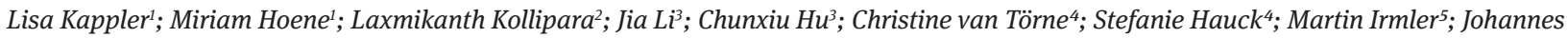
Beckers ${ }^{5}$; Martin Hrabe de Angelis ${ }^{5}$; Andreas Peter ${ }^{1}$; Guowang Xü ${ }^{3}$; Hans-Ulrich Häring ${ }^{1}$; Cora Weigert ${ }^{1}$; Albert Sickmann

${ }^{1}$ University Hospital Tuebingen, Tuebingen, Germany, Clinical Chemistry Central Laboratory, Division of Clinical Chemistry and Pathobiochemistry, Department of Diagnostic Laboratory Medicine, Tübingen, Germany; ${ }^{2}$ Leibniz-Institut für Analytische Wissenschaften - ISAS - e.V., Dortmund, Germany;, Protein Dynamics, Dortmund,Germany; ${ }^{3}$ Dalian Institute of Chemical Physics, Chinese Academy of Sciences, Dalian, China, Key Laboratory of Separation Science for Analytical Chemistry, Dalian,China; ${ }^{4}$ Helmholtz Zentrum München, Deutschland, Research Unit Protein Science, München,Germany; ${ }^{5}$ Helmholtz Zentrum München, German Research Center for Environmental Health (GmbH), 85764 Neuherberg, Germany, Institute of Experimental Genetics, München,Germany; ${ }^{6}$ Leibniz-Institut für Analytische Wissenschaften - ISAS - e.V., Dortmund, Germany;, Protein Dynamics, Dortmund,Germany

Background: Mitochondria are discussed as one of the key players in the pathogenesis of type 2 diabetes. However, experimental evidence is controversial, pointing towards a mitochondrial dysfunction associated with insulin resistance, but also towards compensatory mechanisms. Objectives: Comprehensive investigation of mitochondrial adaptions to high-energy diet (HED) by a multi-omics approach and functional studies to provide new insights into diabetes-associated pathomechanisms in a tissue-specific mitochondrial manner.

Methods: We applied a systems medicine approach combining transcriptomics and lipidomics, proteomics and functional analyses of isolated mitochondria and total tissue to elucidate the interplay between molecular fingerprints and their impact on mitochondrial function. Purified mitochondria (by ultracentrifugation) from skeletal muscle and liver were investigated by LC-coupled proteomic/lipidomic analyses (Q Exactive HF) in a pre-diabetic mouse model (6 weeks of control (10 fat/20 protein/70 carbohydrates [kJ\%]) vs high-energy diet (45 fat/20 protein $/ 35$ carbohydrates $[\mathrm{kJ} \%]$ ), $\mathrm{n}=8$ ). Mitochondrial proteome was covered by non-targeted DIA. Mitochondrial function was investigated by enzymatic assays and high-resolution respirometry, results were validated in human samples. Whole liver transcriptomics was performed. 
Results: More than 22.200 transcripts in whole liver, 950 hepatic mitochondrial proteins and above 540 proteins in isolated muscle mitochondria, as well as around 280 mitochondrial lipid species in both tissue mitochondria were quantified and linked to mitochondrial function. Mitochondria from muscle and liver showed distinctly different lipid and protein profiles, and also different substrate preferences in respirometry. After facing the HED, fatty acid metabolism pathways were enriched, both observed on protein but also transcript level and in fatty acid oxidation in muscle. Various tissue-specific mitochondrial adaptions were observed (assumed to be rescue mechanisms) to avoid substrate flooding of mitochondria, lowering the electron pressure on the electron transport chain and thereby providing protection from cell damage. However, alterations on mitochondrial lipid profile point towards the breakdown of these rescue mechanisms over time.

Conclusions: We linked the mitochondrial molecular composition with tissue-specific mitochondrial (dys)function generating a novel systems medicine tool for elucidating mitochondrial adaptions to e.g. diet-induced insulin resistance. Besides expected mitochondrial alterations due to the diet such as a changed fatty acid metabolism, rather unexpected rescue mechanisms were observed, providing further insights into the pathological remodeling of mitochondria in type 2 diabetes development.

\title{
DGKL-P027
}

\section{Repression of C1QBP precedes pathologic cardiac hypertrophy}

\author{
Eman Hagag; Božena Kesić; Peter Mirtschink \\ Universitätsklinikum Dresden, Institut f. Klinische Chemie und Laboratoriumsmedizin, Dresden, Germany
}

Background and aim: Hypertrophic heart disease is the abnormal thickening of the walls of the left ventricle in response to various systemic or genetically based stress leading to cardiac dysfunction and eventually heart failure. On the cellular level stress-induced signaling cascades trigger a specific transcriptional response largely, mediated by characteristic transcription factors. In addition, various post-transcriptional modifications have been described in the diseased state. In consequence, cardiomyocyte hypertrophy and dysfunction is implemented through fundamental reprogramming of cellular metabolism and energetics. However profound knowledge about the necessary orchestration between transcriptional and posttranscriptional gene regulation is still lacking. Thus, we aim to identify and characterize important RNAbinding proteins (RBPs) that play a key role in this process.

Methods: We have used an integrative in silico approach as well as various in vitro and in vivo analyses to identify central RBPs, with altered expression levels during pressure-overload induced pathologic cardiac hypertrophy. Potential function in growth and contractility, was assessed using mass-spectrometry based metabolomics and various growth assays.

Results: We have identified mitochondrial complement component $1 \mathrm{Q}$ subcomponent-binding protein (C1QBP) as an important RBP in heart disease. Using three different mouse models of cardiac hypertrophy, (i) surgery-induced transaortic constriction (TAC) as a model for pressure-overload induced hypertrophy, (ii) 1 Kidney-1 Clip (1K1C) as a model or renal hypertension and (iii) myocardial infarction (MI) we could demonstrate that C1QBP is consistently repressed on the mRNA and protein level. Strikingly, shRNA mediated repression of C1qb mimicked cardiomyocyte hypertrophy as observed upon alpha- and beta-adrenergic stimulation.

Conclusions: In cardiomyocytes C1QBP abundance is inversely correlated with cellular growth in response to pathologic stress and suspected to play crucial roles in the regulation of RNA splicing, cell metabolism and contractility.

\section{DGKL-P028}

\section{cGMP-dependent Protein Kinase I (cGKI) Modulates Human Hepatic Stellate Cell Activation}

\author{
Andras Franko ${ }^{1}$; Marketa Kovarova ${ }^{2}$; Susanne Feil ${ }^{3}$; Robert Feil ${ }^{3}$; Robert Wagner' ${ }^{1}$ Martin Heni'; Alfred Königsrainer ${ }^{4}$; Marc Ruo $\beta^{5}$; Andreas \\ Nüssler ${ }^{5}$; Cora Weigert ${ }^{1}$; Hans-Ulrich Häring ${ }^{1}$; Stefan Lutz ${ }^{1}$; Andreas Peter ${ }^{1}$ \\ ${ }^{1}$ Universitätsklinikum Tübingen, Innere Medizin IV - Endokrinologie und Diabetologie, Angiologie, Nephrologie und Klinische Chemie, Tübingen, \\ Germany; ${ }^{2}$ Universitätsklinikum Tübingen, Zentrallabor, Tübingen, Germany; ${ }^{3}$ Universität Tübingen, Interfakultäres Institut für Biochemie, \\ Tübingen, Germany; "Universitätsklinikum Tübingen, Allgemeine, Viszeral- und Transplantationschirurgie (AVT), Tübingen, Germany; \\ ${ }^{5}$ Berufsgenossenschaftliche Unfallklinik, Siegfried Weller Institut für Unfallmedizinische Forschung (SWI), Tübingen, Gernany
}

Background Non-alcoholic fatty liver disease and hepatic insulin resistance precede diabetes and may further develop into non-alcoholic steatohepatitis and liver fibrosis/cirrhosis. The activation of hepatic stellate cells (HSC), which is characterized by the loss of retinyl-ester stores, plays a crucial role in the pathophysiology of this process. Since cGMP-dependent protein kinase I (cGKI) deficient (cGKI-KO) mice displayed hepatic insulin resistance and disturbed glucose metabolism, as well as, cGKI is only detected in HSC but not in hepatocytes, we hypothesized that cGKI modulates HSC activation and its metabolic consequences.

Methods First, retinol storage and gene expression were studied in cGKI-KO mice. Second, we investigated the effects of cGKI-silencing on gene expression and cellular activation in the human stellate cell line LX2. Finally, cGKI expression was investigated in human liver biopsies covering a wide range of liver fat content. 
Results Retinyl-ester level in the liver of cGKI-KO mice was lower compared to wild-type animals, which was associated with increased inflammatory gene expression. Activation of LX2 cells showed increased $\alpha$-smooth muscle actin and matrix metalloproteinase 2 expression, which was augmented by silencing cGKI. On the other hand, activation of LX2 cells by TGF $\beta$ suppressed cGKI expression. Furthermore, we detected a negative correlation between cGKI mRNA and liver fat content in human biopsies.

Conclusions The lower mRNA level of cGKI either in TGF $\beta$ activated LX2 cells or in liver biopsies with high fat content suggest that cGKI function might be impaired during HSC activation. Furthermore, the higher expression of inflammatory genes and low retinyl-ester levels in cGKI-KO mice as well as the higher levels of activation markers in cGKI-silenced LX2 cells indicate that cGKI inhibits HSC activation. Since cGKI is a downstream target of nitric oxide (NO), it is conceivable that NO may exert protective effects inhibiting liver fibrosis via cGKI.

\section{DGKL-P029}

\section{Gender differences in the predictive value of biomarkers in end-stage liver disease}

Johannes Remmler'; Joachim Thiery²; Thorsten Kaiser ${ }^{2}$

${ }^{1}$ Institut für Laboratoriumsmedizin, Universitätsklinikum Leipzig, Klinische Chemie/Immunologie/Liquordiagnostik/Endokrinologie, Leipzig, Germany; Institut für Laboratoriumsmedizin, ${ }^{2}$ Universitätsklinikum Leipzig, Institut für Laboratoriumsmedizin, Leipzig, Germany

BACKGROUND: Organ allocation for liver transplantation is based on the MELD score for estimation of prognosis. The MELD score is calculated from biomarkers, namely bilirubin, INR and creatinine. The MELD score applies equally for women and men although there are known gender differences in these biomarkers.

OBJECTIVE: Based on a large clinical cohort, we quantified gender differences of several biomarkers and their predictive values in end stage liver disease.

MATERIALS AND METHODS: We performed a retrospective observational cohort study of 774 patients with end-stage liver disease (62.4\% male, median follow-up time 335 days), evaluated for liver transplantation at the Leipzig University Hospital.

RESULTS: The most relevant biomarkers with significant gender differences where creatinine (median: $\delta 83 \mu \mathrm{mol} / \mathrm{l}$,, $71 \mu \mathrm{mol} / 1$ [p $<0.001]$ ) and the MELD score $(\delta 11.5,+10.2[\mathrm{p}=0.007])$. Considering only the patients who died during follow-up without receiving an organ $(\mathrm{n}=110)$, MELD score showed a different tendency and doesn't differ significantly $(\delta 19.0$, $q$ 20.7; $[\mathrm{p}=0.212])$. Among these patients, men showed a

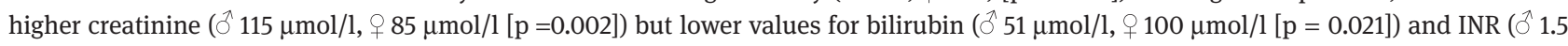
$\mu \mathrm{mol} / 1$, $+1.7 \mu \mathrm{mol} / 1[\mathrm{p}=0.014])$. The predictive value of creatinine for mortality without transplantation was higher among men compared to women (area under ROC [95\%-CI]: $\lesssim 0.71$ [0,63-0,79], 0.62 [0,51-0,72]). However for other biomarkers the predictive power was higher among

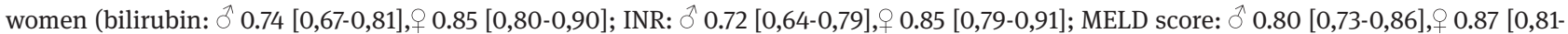
0,92]). Cystatin C predicted mortality better than creatinine and equally for women and men ( $\overbrace{}^{\lambda} 0.75$ [0,68-0,82],, 0.75 [0,68-0,82]).

DISCUSSION: The study showed substantial gender differences in the prediction of mortality, with relevance for organ allocation. Median creatinine levels as well as the predictive value of creatinine were significantly higher among men. Contrastingly, bilirubin and INR levels of patients who died without transplantation were significantly higher among women. The predictive values of bilirubin, INR and MELD score regarding mortality without transplantation were also higher among women. Furthermore, the study revealed cystatin $\mathrm{C}$ as a better mortality predictor, compared to creatinine, especially for women, where the predictive value of creatinine is limited.

CONCLUSION: There are gender specific differences in predicting mortality in end-stage liver disease patients. To prevent disadvantage for one sex, MELD score could be calculated specifically for the genders to improve mortality prediction and fairness in organ allocation. Moreover, the use of cystatin $\mathrm{C}$ instead of creatinine could improve mortality prediction, especially in women.

\section{DGKL-P030}

\section{Quantitation of Trimethylamine N-oxide and its precursors by HILIC-MS/MS and their potential role in cognitive behavior}

Alexander Gaudl; Benedikt Zöhrer; Madlen Reinicke; Uta Ceglarek

Universitätsklinikum Leipzig, Institut für Laboratoriumsmedizin, Klinische Chemie und Molekulare Diagnostik, Leipzig, Germany

Introduction: Trimethylamine N-oxide (TMAO), though already known to marine biologists for many years, recently found itself in the scientific spotlight through its association with cardiovascular disease (CVD). Because it affects cholesterol and bile acid metabolism, further association with cognitive disorders is assumable. Results from previous studies focusing on the cardiovascular angle were inconclusive regarding TMAOs role as a biomarker for CVD and studies concerning cognitive functioning are virtually absent. Furthermore, baseline TMAO levels appear to differ significantly between populations from different continents. We established a new HILIC-MS/MS method and determined normal ranges of TMAO and its precursors Betaine, Carnitine, and Choline by means of the LIFE Adult study $(\mathrm{n}=1000)$. Moreover, a potential association with cognitive behavior was assessed using SISCO score. 
Methods: Plasma or serum $(10 \mu \mathrm{l})$ was prepared by protein precipitation using $90 \mu \mathrm{l}$ methanol including internal standards. Prior to tandem mass spectrometric analysis $10 \mu \mathrm{l}$ of the supernatant was diluted with $990 \mu \mathrm{l}$ Acetonitrile/0.2 M ammonium formate buffer 90/10 v/v representing the initial conditions of the chromatographic separation. Rapid hydrophilic interaction liquid chromatography (Kinetex@ HILIC $50 \times 2.1 \mathrm{~mm}$, Phenomenex) was coupled to tandem mass spectrometric detection on a SCIEX QTRAP® 6500, applying electrospray ionization in positive ion mode. Injection volume was $5 \mu$ land the total run time was 5 minutes. Deuterium-labeled analytes were used as internal standards. Results: Between-run imprecision ranged from $4.7 \%$ to $6.8 \%$ and LLOQ were below $0.3 \mu \mathrm{M}$ at thousandfold dilution. Recovery rates ranged from $97 \%$ to $102 \%$. Preanalytical investigations regarding hands on time of whole blood, blood plasma and processed samples at different temperatures, as well as stability towards freezing and thawing of biological samples or influence of sample material showed high stability and low variance in general. Solely choline concentration showed a slight increase during storage of whole blood at room temperature. Preliminary base data for LIFE Adult gave median TMAO concentrations of $3.8 \mu \mathrm{mol} / \mathrm{l}$ (IQR: $2.85 .5 \mu \mathrm{mol} / \mathrm{l}$ ).

Conclusion: We present a robust and highly reproducible HILIC-MS/MS method for the quantitation of TMAO and its precursors Betaine, Carnitine, and Choline. Furthermore, it proved its reliability being applied to 1000 samples of the LIFE Adult study.

\section{DGKL-P031}

\section{Associations of a panel of circulating adipokines with fat deposits, metabolic risk factors, and renal function in a general population}

Stephanie Zylla ; Henry Völzke²; Jan Kassubek ${ }^{3}$; Jens-Peter Kühn ${ }^{4}$; Matthias Nauck ${ }^{1}$; Nele Friedrich ${ }^{1}$

${ }^{1}$ Universitätsmedizin Greifswald, Institute for Clinical Chemistry and Laboratory Medicine, Greifswald, Germany; ${ }^{2}$ University Medicine Greifswald, Germany, Institute for Community Medicine, Greifswald, Germany; ${ }^{3}$ University of Ulm, Department of Neurology, Ulm, Germany; ${ }^{4}$ University Medicine Greifswald, Germany, Department of Diagnostic Radiology and Neuroradiology, Greifswald

BACKGROUND: Adipose tissue is known to secrete a multitude of bioactive adipokines and it seems that the majority of these is implicated in the pathogenesis of obesity and associated metabolic diseases. Existing research studies mostly concentrated on one or two adipokines and therefore, comparisons of the involvement of different adipokines in these disorders are difficult. Furthermore, associations of adipokine concentrations with fat deposits or behavioral risk factors are mostly missing. This study aimed to provide a comprehensive overview of the associations of a panel of adipokines (adiponectin, chemerin, galectin-3, leptin, resistin) with magnetic resonance imaging (MRI)-quantified fat deposits, behavioral risk factors, metabolic parameters, blood pressure, and renal function in a large population-based study.

METHODS: Data from 4,114 subjects of the Study of Health in Pomerania were analyzed. Residual method was used to investigate the associations of MRI-quantified liver fat content as well as visceral (VAT) and subcutaneous adipose tissue (SAT) with circulating adipokine concentrations. Multivariable regression models were applied to examine the associations of adipokine concentrations with behavioral risk factors, metabolic phenotypes, blood pressure, renal function as well as metabolic syndrome and its components.

RESULTS: Independently of other fat compounds, liver fat content, SAT, and VAT were inversely associated with adiponectin. An independent positive association of liver fat content and SAT with chemerin was observed. The strongest independent association was detected between SAT and leptin. Physically inactive subjects had higher chemerin and leptin concentrations. Smokers had higher chemerin and galectin-3 as well as lower adiponectin and leptin concentrations. Alcohol consumption was associated with adiponectin (positive) and resistin (inverse). Obese subjects showed higher chemerin, galectin-3, and leptin concentrations, but lower adiponectin concentrations. All adipokines were associated with at least on lipid marker. Associations with parameters of glucose metabolism were seen for adiponectin, chemerin, galectin-3, and leptin. With the exception of adiponectin, adipokines were inversely associated with estimated glomerular filtration rate to a similar strength.

CONCLUSION: Our results indicate that the associations between different adipokine concentrations and metabolic risk factors as well as renal function significantly differ. In general, high adiponectin concentrations seem to have a protective role whereas high chemerin, galectin-3, leptin, or resistin concentrations seem to be disadvantageous. Leptin concentrations are strongly associated with abdominal obesity, whereas adiponectin and chemerin concentrations showed strong associations with widespread metabolic disorders. Four of the five adipokines were related to renal function which should be considered in future investigations on adipokine concentrations.

\section{Posterwalk Endokrinologie, Stoffwechsel und Herz/Kreislauf II}

\section{DGKL-P032}

\section{Plasma chemerin concentrations are inversely related to bone quality in obesity}

Lejla Kadric; Stephanie Zylla; Matthias Nauck; Nele Friedrich; Anke Hannemann

Universitätsmedizin Greifswald, Institut für Klinische Chemie und Laboratoriumsmedizin, Greifswald, Germany

Background: Chemerin is an adipokine associated with parameters of inflammation and the metabolic syndrome. Small observational studies suggested that high circulating chemerin levels are also related to bone erosion. We aimed to determine whether plasma 
chemerin levels are related to bone quality in the general population and to investigate the influence of body mass index (BMI) on that relation.

Material and Methods: Data from 3,583 adults who participated in the population-based Study of Health in Pomerania (SHIP)-Trend were obtained. The participants were divided into three groups according to their BMI: lean $\left(<25 \mathrm{~kg} / \mathrm{m}^{2}\right)$, overweight $\left(25-30 \mathrm{~kg} / \mathrm{m}^{2}\right)$, and obese $(\geq$ $30 \mathrm{~kg} / \mathrm{m}^{2}$ ). Chemerin concentrations were determined in EDTA plasma (Mediagnost, Reutlingen, Germany). Bone quality was assessed using quantitative ultrasound at the heel (Achilles InSight, GE Healthcare, U.K.). The broadband ultrasound attenuation (BUA), speed of sound (SOS), stiffness index, and the osteoporotic fracture risk were derived from this measurement. Sex-and BMI-specific linear regression models adjusted for age, physical activity, smoking, diabetes mellitus, HbA1c, eGFR, fibrinogen and BMI were calculated to assess the associations between plasma chemerin levels and the QUS parameter. Logistic regression models with the same adjustment set were calculated to analyze the association between chemerin levels and a medium/high osteoporotic fracture risk.

Results: About $78 \%$ of men and $60 \%$ of women in our study population were overweight or obese. With respect to plasma chemerin levels, a strong increase over BMI groups was observed in both sexes. The linear regression models revealed inverse associations between plasma chemerin levels and BUA in obese men. In obese women inverse relations between plasma chemerin levels and SOS or stiffness index were found. Logistic regression models confirmed these observations as positive associations between plasma chemerin and the osteoporotic fracture risk were detected. In lean or overweight subjects no statistically significant associations were found.

Conclusion: Our sex- and BMI-specific analyses showed that inverse associations between plasma chemerin levels and bone quality are restricted to obese men and women. The observed association may be due to a chemerin-induced negative affect on bone metabolism, possibly due to abrogation of osteoblastogenesis or stimulation of adipogenesis.

\title{
DGKL-P033
}

\section{Acylated ghrelin and unacylated ghrelin in subjects at risk of type $\mathbf{2}$ diabetes mellitus and alterations (of acylation) during oral glucose tolerance tests}

Magnus Wolf ${ }^{1}$; Martin Heni' ${ }^{1}$ Anita M. Hennige ${ }^{2}$; Joachim Stangier ${ }^{2}$; Hans-Ulrich Häring ${ }^{1}$; Andreas Fritsche ${ }^{1}$; Andreas Peter ${ }^{1}$

${ }^{1}$ University of Tübingen, Department of Internal Medicine IV, Division of Endocrinology, Diabetology, Vascular Medicine, Nephrology and Clinical Chemistry, Tübingen, Germany; ${ }^{2}$ Boehringer Ingelheim, International GmbH, Biberach, Germany

The gastric orexigenic peptide hormone ghrelin has been implicated in the pathophysiology of obesity and type 2 diabetes mellitus with a unique posttranslational acyl modification regulating its biochemical activity and translocation into the central nervous system. Modulation of ghrelin levels as well as acylation may therefore be promising pharmacological targets.

In this study we aimed to investigate the relation of acylated (AcG) as well as unacylated ghrelin (UnG) with body weight and glucose homeostasis in the fasting ( $\mathrm{n}=545$ subjects) and post-oral glucose tolerance test (oGTT) state ( $\mathrm{n}=280$ subjects) in a cohort covering a broad range of BMI $\left(17.95 \mathrm{~kg} / \mathrm{m}^{2}-76.25 \mathrm{~kg} / \mathrm{m}^{2}\right)$.

Baseline median (25th percentile; 75th percentile) levels of AcG and UnG were $94.2 \mathrm{pg} / \mathrm{ml}$ (53.5 pg/ml; $154.9 \mathrm{pg} / \mathrm{ml})$ and $175.3 \mathrm{pg} / \mathrm{ml}$ (97.3 pg/ $\mathrm{ml} ; 337.4 \mathrm{pg} / \mathrm{ml})$ with an $\mathrm{AcG} / \mathrm{UnG}$ ratio of 0.48 (0.34; 0.75).

Fasting AcG $(\mathrm{p}<0.0001, \mathrm{r}=-0.22)$ and UnG $(\mathrm{p}<0.0001, \mathrm{r}=-0.33)$ were negatively and the AcG/UnG ratio was positively ( $\mathrm{p}<0.0001$, $\mathrm{r}=+0.19)$ correlated with BMI. Matsuda's insulin sensitivity index (ISI) correlated positively with AcG $(\mathrm{p}=0.0014, \mathrm{r}=+0.14)$ and UnG $(\mathrm{p}=0.0004, \mathrm{r}=+0.15)$ but not with the AcG/UnG ratio $(\mathrm{p}=0.2978)$. In a multivariate analysis including BMI and ISI, all correlations remained significant for BMI $(\mathrm{p}<0.0001)$ but only the AcG/UnG ratio for ISI $(\mathrm{p}=0.0333)$.

During an oGTT, AcG and UnG showed slight decreases at early (UnG after $30 \mathrm{~min}: \mathrm{p}=0.0321,-6.8 \%$ ) and increases at later time points (AcG after 90 min: $p=0.0009,+13.6 \%$; AcG after $120 \mathrm{~min}: \mathrm{p}=0.0158,+14.9 \%)$. AcG/UnG ratio increased after $60 \mathrm{~min}(\mathrm{p}=0.0164,+9.4 \%)$ and $90 \min (\mathrm{p}=0.0086,+12.1 \%)$.

Stratification of the subjects into low BMI $\left(<30 \mathrm{~kg} / \mathrm{m}^{2}\right)$, medium BMI $\left(30 \mathrm{~kg} / \mathrm{m}^{2}-40 \mathrm{~kg} / \mathrm{m}^{2}\right)$ and high BMI $\left(>40 \mathrm{~kg} / \mathrm{m}^{2}\right)$ groups revealed more pronounced changes during oGTTs in the low and medium BMI groups.

In conclusion, our data demonstrates a downregulation of both AcG and UnG in obesity with an increased AcG/UnG ratio. Post-oGTT changes in ghrelin are modest in lean and blunted in obese human subjects.

\section{DGKL-P034}

\section{The role of MFGE8 in obesity-related metabolic dysregulation}

\author{
Antonios Chatzigeorgiou; David Haddad; Marina Nati; Kyoung-Jin Chung; Triantafyllos Chavakis \\ TU Dresden Institut für klinische Chemie und Laboratorium, Institut für Klinische Chemie und Laboratoriumsmedizin, Dresden, Germany
}

During the course of obesity, the adipose tissue is beset by a number of pathological dysregulations, including insulin resistance, low grade inflammation, and lipodystrophy. Milk fat globule-EGF-factor 8 (MFG-E8) - a glycoprotein well-characterized in opsonophagocytosis - is a 
factor, which is highly responsively upregulated during diet-induced obese conditions. Using an MFG-E8 deficient mouse model, we aimed to elucidate the role of this protein in the context of a number of processes which are critically modulated in diet-induced obese conditions; including metabolic, immunological, angiogenic and adipogenic adaptations. We found that high-fat diet fed Mfg-e8-/- mice are protected from the insulin resistance and inflammatory phenotype of the obese adipose tissue compared with wild-type counterparts, resulting furthermore in ameliorated systemic insulin sensitivity and dyslipidemia - with no phenotypic differences being observed under a corresponding normal diet feeding. Conversely, however, MFG-E8 deficient mice presented with an impaired capacity of adipocytes to expand in respond to high lipid uptake, and countervailed by increased cell proliferation, while total adipose tissue weight and vascularization was unchanged. Therefore, we propose that MFG-E8 helps to positively regulate the programming events, which drive these processes in the context of obesity, and thereby leads to the exacerbation of high fat diet-induced metabolic dysregulation by abetting excessive adipocyte hypertrophy.

\title{
DGKL-P035
}

\section{Free 25-hydroxyvitamin D in normal human pregnancy}

\author{
Oleg Tsuprykov' ${ }^{1}$ Claudia Buse $^{1}$; Roman Skoblo ${ }^{1}$; Berthold Hocher ${ }^{2}$ \\ ${ }^{1}$ IFLb Laboratoriumsmedizin Berlin GmbH, Berlin, Germany; ${ }^{2}$ Hunan Normal University, Changsha, China; University of Potsdam, Potsdam, \\ Germany, Key Laboratory of Study and Discovery of Small Targeted Molecules of Hunan Province, Potsdam,
}

Low vitamin D in pregnancy may lead to a wide range of adverse health outcomes in offspring. 25-hydroxyvitamin D (250HD) is a routine marker of vitamin D status. Since circulating 25OHD is mostly bound to vitamin D-binding protein (DBP) and DBP is elevated during pregnancy, it is doubtful whether $250 \mathrm{HD}$ levels adequately reflect vitamin D status in pregnancy. We aimed to: (1) compare the correlation strength between free 250HD and markers of bone health as well as other markers with total 25OHD; (2) compare two methods of free 250HD determination - a direct measurement and calculation via formula; (3) establish reference intervals for free 250HD. The study participants were 368 Caucasian pregnant healthy women residing in Berlin, Germany. Total 250HD (250HDt) was assessed by Abbott Architect platform. Directly measured free 250HD (250HDm) was determined by an ELISA kit by Future Diagnostics (Netherlands). DBP was measured by an ELISA with polyclonal antibodies (Immundiagnostik, Germany). Calculated free 250HD (250HDc) was determined using the formula based on the laws of protein-ligand binding kinetics. Spearman rank correlation analysis was used (Spearman's rho ( $\rho)$ ). Calcium positively correlated with free 250HD ( $\rho=0.141, p=0.007$ and $\rho=0.237, p<0.001$ for 250HDm and 250HDc, respectively), but not with 250HDt ( $\rho=0.060, p=0.251$ ). All 25OHD isoforms negatively correlated with PTH $(\rho=-0.138, p=0.008 ; \rho=-0.196, p<0.001 ; \rho=-0.227, p<0.001$ for 25OHDm, 250HDc, and $25 \mathrm{OHDt}$, respectively). Although all 250HD isoforms negatively correlated with BSAP, the association strength was higher with free 25OHD isoforms ( $\rho=-0.203, p<0.001 ; \rho=-0.211, p<0.001 ; \rho=-0.108, p=0.038$ for 250HDm, 25OHDc, and 25OHDt, respectively). Adiponectin positively correlated with free 250HD ( $\rho=0.142, p=0.008$ and $\rho=0.161, p=0.003$ for 250HDm and 25OHDc, respectively), but not with 250HDt ( $\rho=0.054$, $p=0.307$ ). Urea positively correlated with free 250HD ( $\rho=0.114, p=0.032$ and $\rho=0.149, p=0.005$ for 25OHDm and 25OHDc, respectively), but not with 25OHDt $(\rho=0.008, p=0.887)$. Free thyroxine, vitamin B12 and low-density lipoprotein (LDL) correlated with 250HDm and 250HDc (negatively with thyroxine and vitamin B12, and positively with LDL), but not with 250HDt. High-density lipoprotein correlated (positively) with 250HDt, but not with free 250HD isoforms. The two methods of free 250HD determination showed strong correlation with each other $(\rho=0.858, \mathrm{p}<0.001)$. The proposed reference intervals for 250HDm are 1.74-8.00, 1.11-7.41, and 1.20-6.51 pg/mL and for 250HDc are 0.84-6.62, $0.79-5.91$, and $0.49-6.20 \mathrm{pg} / \mathrm{mL}$ for 1st, 2nd, and 3rd trimesters, respectively. Free 250HD showed better correlation with markers of bone health compared to total 25OHD. Optimal monitoring of vitamin D status in pregnancy in addition to total 25OHD should include free 25OHD determination. Both way of free $250 \mathrm{HD}$ determination can be applied. We established trimester-specific reference intervals for free $250 \mathrm{OH}$ in normal pregnancy.

\section{DGKL-P036}

\section{Increased hepatic cholesterol biosynthesis due to Abcc6 deficiency in mice}

\author{
Bettina Ibold ${ }^{1}$;ta Ceglarek ${ }^{2}$; Julia Dittrich²; Janina Tiemann ${ }^{1}$; Cornelius Knabbe ${ }^{1}$; Doris Hendig ${ }^{1}$ \\ ${ }^{1}$ Herz- und Diabeteszentrum Nordrhein-Westfalen, Universitätsklinik der Ruhr-Universität Bochum, Institut für Laboratoriums- und \\ Transfusionsmedizin, Bad Oeynhausen, Germany; \\ ${ }^{2}$ Universitätsklinikum Leipzig, Institut für Laboratoriumsmedizin, Klinische Chemie und Molekulare Diagnostik, Leipzig, Germany
}

Objectives: Pseudoxanthoma elasticum (PXE) is a metabolic disease characterized by progressive connective tissue calcification of elastic fibers due to mutations in the adenosine triphosphate-binding cassette transporter, subfamily C, member 6 (ABCC6) gene. Genetic studies link sequence variations of ABCC6 to altered high-density lipoprotein(HDL) cholesterol and triglyceride levels and an elevated risk for coronary artery diseases. However, the role of ABCC6 in cholesterol homeostasis is largely unknown. Therefore, we analyzed cholesterol biosynthesis activity and proprotein convertase subtilisin/kexin type 9 (Pcsk9) expression in Abcc6 deficient mice and in PXE patients. 
Methods: We quantified serum concentration of cholesterol precursors lanosterol, zymosterol/desmosterol/7-dehydrocholesterol (zy/ de/7dhc), cholesterol as well as campesterol and $\beta$-sitosterol in 6-month-old and 12-month-old wildtype (WT) and Abcc6-/- mice to assess cholesterol biosynthesis activity. Moreover, we performed quantitative real time PCR with murine liver tissue samples for analyzing relative gene expression of 3-hydroxy-3-methylglutaryl-coenzyme A reductase (Hmgcr), farnesyl pyrophosphate synthase (Fdps), farnesyl-diphosphate farnesyltransferase 1 (Fdft1), geranylgeranyl pyrophosphate synthase 1 (Ggps1), lanosterol synthase (Lss), low-density lipoprotein receptor (Ldlr) and Pcsk9. In addition, we determined Pcsk9 protein serum levels in these mice as well as in PXE patient serum compared to healthy controls.

Results: Here, we report that Abcc6 deficiency in mice leads to decreased free, esterified as well as total cholesterol and phytosterol serum levels up to $45 \%$ in both 6- and 12-month-old Abcc6-/- mice compared to WT mice. Furthermore, we found increased ratios of zy/de/7dhc to total cholesterol serum level in both ages of Abcc6 deficient mice. Hepatic Hmgcr and Ggps1 gene expression tended to be enhanced in 6- and 12-month-old Abcc6-/- mice relative to WT mice. By contrast, transcript levels of Fdft1 were significantly downregulated by $30 \%$ in liver tissue of 12-month-old Abcc6-/- mice, whereas Fdft1 expression exhibited a 1.8-fold increase in younger Abcc6-/- mice in comparison to age matched WT mice. We detected an elevated Pcsk9 serum concentration in both Abcc6 knockout mice and PXE patients in comparison to appropriate controls. However, gene expression of Pcsk9 in liver tissue of Abcc6-/- mice was not altered.

Conclusion: In summary, our findings underline an activated cholesterol biosynthesis due to an Abcc6 deficiency in younger and older mice. We also showed an elevated Pcsk9 serum level in both Abcc6 knockout mice and PXE patients. These results consequently indicate impaired cholesterol metabolism as important mechanism involved in pathophysiology of PXE.

\title{
DGKL-P037
}

\section{Characterization of CD248 (TEM1) during high glucose induced mesangial dysfunction}

Ronald Biemann'; Shruthi Krishnan²; Sophie-Cecil Mathieu²; Jayakumar Manoharan²; Hongjie Wang²; Berend Isermann² ${ }^{1}$ Institut für Klinische Chemie und Pathobiochemie, Endokrinologie, Magdeburg, Germany; ${ }^{2}$ Otto-von-Guericke-Universität Magdeburg, Institut für Klinische Chemie und Pathobiochemie, Magdeburg, Germany

Aim of the study: Diabetic Nephropathy (dNP) is the most common cause of chronic kidney disease worldwide and a growing public health challenge. Progress of dNP is promoted by dysfunctional mesangial cells in the glomerular compartment that show increased cellular expansion, extracellular matrix production and maladaptive cellular crosstalk with epithelial and endothelial cells. Aim of our study is to investigate the role of the pericyte specific transmembrane receptor TEM1 in mesangial cells that are exposed to diabetic conditions in vivo and in vitro. Methods and results: We compared the glomerular expression of TEM1 in diabetic and non-diabetic mice. Furthermore, we analyzed glomeruli of TEM1-/- mice after 26 weeks of streptozotocin induced diabetes, showing that loss of TEM1 protects mice from diabetes induced glomerular fibrosis. We overexpressed and silenced TEM1 in murine mesangial cells in vitro to analyze how TEM1 interacts with different pathways that may promote mesangial expansion and mesangial extracellular matrix production. We found that knock down of TEM1 prevents high glucose induced activation of unfolded protein response (UPR), Smad2/3 phosphorylation and mTORC1 activation by phosphorylation of mTOR, Raptor and its downstream effector p70S6 kinase. These results were validated in primary murine mesangial cells. We further identified HSP90 as binding partner of TEM1 by FLAG immunoprecipitation. HSP90 has been shown to control UPR.

Conclusion: High glucose induced mesangial TEM1 expression promotes mesangial dysfunction in vivo and in vitro by activation of UPR and mTORC1. Hence, targeting mesangial TEM1 expression by therapeutic interventions may slow down, prevent or even reverse diabetes induced glomerular fibrosis.

\section{DGKL-P038}

\section{Characterization and Potential Clinical Application of a Novel Test System for the Quantification of Histamine Degradation Activity in Humans}

\author{
Christian Lutz'; Gabrielle Frost ${ }^{1}$; Thomas Knittel' ${ }^{1}$; Albert Missbichler ${ }^{2}$ \\ ${ }^{1}$ Frost Diagnostika, F\&E, Otterstadt, Germany; ${ }^{2}$ LLDORM Consulting Group, F\&E, Wien, Austria
}

\begin{abstract}
Aims: Histamine intolerance (HIT) is a multifaceted clinical condition triggered by histamine-rich foods, alcohol and/or by drugs that liberate histamine or block diamine oxidase (DAO), the main enzyme involved in the metabolism of ingested histamine. The aim of the study was the development of an assay measuring the total histamine degrading capacity (THDC) in an easily accessible human specimen such as serum independent of the patient-specific nutritional status and to compare results with state-of-the-art assays (DAO REA) as well as clinical data. Methods: The FD THAK/THDC test quantitatively determines the capability to degrade histamine in human serum samples irrespective of the way of degradation. Samples were incubated with a histamine-containing- provocation solution and histamine levels before and after provocation were determined with the THAK/THDC ELISA. Values are expressed as \% histamine degrading capacity based on the histamine levels
\end{abstract}


before and after provocation. A 0-25\% degradation was regarded to indicate insufficient or low total histamine degrading capacity (THDC), the $25-40 \%$ range to indicate limited THDC and values $>40 \%$ as normal THDC. THDC serum values were compared to total DAO serum levels (DAO ELISA) and DAO activity as determined by the DAO REA assay.

Results: In 36 patients with a clinically defined HIT diagnosis insufficient of low THDC levels ( $<25 \%$ degradation) were detected in $94 \%$ of cases $(n=34)$, in 2 patients THDC was between $25-40 \%$ and no cases of a THDC $<40 \%$ were evident. In these 36 HIT patients DAO REA levels below $3 \mathrm{U} / \mathrm{ml}$ were detectable in $20 \%$ of cases only $(\mathrm{n}=7)$, between $3-10 \mathrm{U} / \mathrm{ml}$ in 24 pts and $>10 \mathrm{U} / \mathrm{ml} \mathrm{in} 5$ cases. DAO serum levels were below $3 \mathrm{U} / \mathrm{ml}$ in $2 \mathrm{pts}$, between $3-10 \mathrm{U} / \mathrm{ml}$ in $28 \mathrm{pts}$ and $>10 \mathrm{U} / \mathrm{ml}$ in $6 \mathrm{pts}$. In this HIT patient cohort there was a correlation between a pathological THDC and DAO REA test (7/7 pts) and vice versa (7/33 pts.)

In patients with clinical symptoms after food uptake regarded as food hypersensitivity ( $\mathrm{n}=21)$ a THDC value below $25 \%$ was not detectable, THDC values between 25-40\% were present in 19 cases and $>40 \%$ in 2 cases. In this group DAO REA levels were below $3 \mathrm{U} / \mathrm{ml}$ in 0 cases, between $3-10 \mathrm{U} / \mathrm{ml}$ in 11 cases and $>10 \mathrm{U} / \mathrm{ml}$ in 10 cases, while DAO serum levels were below $3 \mathrm{U} / \mathrm{ml}$ in 0 pts, between 3-10 U/ml in 10 pts and $>10 \mathrm{U} / \mathrm{ml}$ in 11 pts.

In control patients $(n=62)$ a pathological THDC was not detectable and all patients had levels $>40 \%$ total histamine degrading capacity, while borderline DAO REA values (3-10 U/ml) were evident in 3 patients and in 14 pts for borderline DAO serum levels (3-10 U/ml).

Conclusions: The easy to use FD THAK/THDC test is a promising novel assay system detecting more cases of histamine intolerance than the currently employed standard marker DAO REA and DAO ELISA. The test could be a valuable diagnostic tool for the identification and characterization of patients with clinical symptoms related to impaired histamine metabolism.

\title{
DGKL-P039
}

\section{Inhibition of BRD4 by JQ1 and RVX-208 rescues endothelial cell dysfunction in an in vitro model of atherosclerosis}

Sidra Shahid

Universität Göttingen, Institute of Clinical Chemistry, Göttingen, Germany

Endothelial cell (EC) monolayer lines the inner lining of vascular system and maintains vessel homeostasis. EC dysfunction, caused by cardiovascular risk factors, is one of the major contributing factors in development of atherosclerosis. During EC dysfunction, EC becomes activated

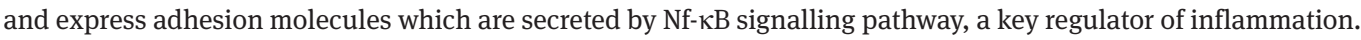

Bromodomain Protein 4 (BRD4) is a transcriptional regulator which is important for multiple cellular activities such as inflammation, DNA damage repair, spermatogenesis, and cancer. Recently it has been shown that BRD4 redistributes from basal cell state maintaining gene loci to super enhancers of inflammatory gene loci during EC dysfunction and binds to NfkB. There are two naturally occurring splice variants/ isoforms of BRD4, named as long and short isoforms. These two isoforms are shown to have opposite roles in breast cancer progression. In the present study, we systematically analysed the role of BRD4 isoforms in EC dysfunction and investigated whether BRD4 inhibition restores EC function under inflammation-stimulating conditions. Towards this end, we treated Human Umbilical Cord derived Vascular Endothelial Cells (HUVECs) with TNF $\alpha$ and established an in vitro model of inflammatory phenotype. We observed overexpression of both BRD4 isoforms upon TNF $\alpha$ treatment to HUVECs, while pre-treatment with BRD4 inhibitors (JQ1 and RVX-208) abrogated the observed overexpression and inflammatory phenotype. We also observed that HUVECs treated with TNF $\alpha$ showed disrupted monolayer integrity and increased permeability, while HUVECs pre-treated with JQ1 or RVX-208 retained their monolayer integrity. We also analysed the expression of Midkine, a small cytokine molecule and an indicator of disrupted monolayer integrity, which has elevated serum levels in human and mice with atherosclerotic plaques. When HUVECs were exposed to TNF $\alpha$ for 72 hours they secreted Midkine, while the cells pre-treated with BRD4 inhibitors showed significantly reduced secretion of Midkine. Collectively, our results indicate that BRD4 inhibition reduces EC dysfunction, however further studies aimed to study the potential of BRD4 inhibition as a therapeutic option of atherosclerosis are warranted.

\section{Posterwalk Onkolgie, Hämatologie und Hämostaseologie I}

\section{DGKL-P040 / PV06}

\author{
Human Plasma Fibronectin as a DNA-Binding Protein \\ Maximilian Strauß; Maren Hedtke; Victor Costina; Michael Neumaier \\ Medical Faculty Mannheim of Heidelberg University, Institute for Clinical Chemistry, Mannheim, Germany
}

Background: Circulating free DNA (cfDNA) is an emerging diagnostic and prognostic biomarker in neoplastic diseases. Since at least part of the cfDNA in an individual suffering from a neoplastic disease originates from tumor cells and contains potentially the whole tumor genome, 
it is a promising target for tumor genotyping. Accordingly, correlations between genetic alterations in the cfDNA could already be demonstrated for some tumor entities. Moreover, several studies were able to identfy other parameters of the cfDNA such as the methylation status, its global concentration and its fragmentation which showed tumor-associated changes and thus might also be of clinical importance in the future.

Although data regarding the generation of cfDNA is quite abundant, very little is known about its stability in and clearance from the bloodplasma, and which pre-analytical variables might be of importance. Our previous research suggests that one of the factors influencing cfDNA stability is the extent of its methylation. If so, this effect may be mediated by a yet undescribed interaction with a plasma protein. Our goal in the present study is to identify plasma proteins binding to cfDNA dependent on its methylation state.

Materials and Methods: We attached both in-vitro methylated and unmethylated amplicons to magnetic streptavidin beads and incubated them in blood plasma using serial dilutions with PBS to detect low-affinity specific differences in the affinity of potential interactions. The amplicons were derived from the RASSF1A promotor region, from genomic DNA isolated from human Melanoma cells. That region was identified as a site exhibiting varying degrees of hypermethylation in melanoma patients. Additionally, as a control for potential unspecific binding, an amplicon from an intron of the same gene was used

After incubation of the bead-amplicon complex in plasma and several washing steps we detached the beads from the amplicons using restriction enzymes and magnetically removed the beads. The remaining supernatant containing the amplicon and eventually bound proteins was then analysed using mass spectrometry.

Results: Plasma Fibronectin was identified as a protein binding to both methylated and unmethylated amplicons from the promotor region as well as the intronic amplicon. Serial dilutions of the plasma with PBS showed no significant difference between any of the amplicons.

Unspecific binding of Fibronectin to the beads was excluded using empty beads without bound DNA.

Discussion: Human plasma Fibronectin has long been known to potentially have DNA binding properties. However, we provide the first evidence of the relevance of this interaction in vivo and thus propose a new role for Fibronectin as a DNA-binding protein in plasma. While our results suggest that this binding is unspecific and independent of the methylation status or other characteristics of the DNA such as binding sites etc., further quantification of this interaction is warranted.

\title{
DGKL-P041
}

\section{Establishing a method for isolation and purification of exosomes}

\author{
Sara Alameldin ${ }^{1}$; Michael Neumaier ${ }^{2}$; Maren Hedtke ${ }^{2}$ \\ ${ }^{1}$ Medizinische Fakultät Mannheim, Universität Heidelberg, Institut für Klinische Chemie, Mannheim, Germany; ${ }^{2}$ Uniklinikum Mannheim, Institut \\ für Klinische Chemie, Mannheim, Germany
}

Introduction: Exosomes are small, lipid bilayer membrane vesicles, 30-100 nm in diameter, secreted by various cell types and found in all body fluids. These vesicles, loaded with unique RNA and protein cargo, have a wide range of biological functions, including cell-to-cell communication and signaling in both normal physiology and disease states, particularly cancer.

Many aspects of exosomes present them as novel means to identify cancer biomarkers for early detection and as therapeutic targets, and using intrinsic and engineered characteristics of exosomes as therapeutic devices to ameliorate the progression of the disease.

Isolation of vesicles from plasma with good recovery and without contamination of proteins and lipoproteins, however, is a challenge.

Lipoproteins are main contaminants in most of the procedures for isolating exosomes. By isolating exosomes using size exclusion chromatography (SEC) we can deplete most of the HDL and LDL. However, exosomes are still not pure enough for identifying the broadly accepted exosomal markers (e.g. tetraspanins) by Mass Spectrometry. One of the ways to precipitate lipoproteins is using Phosphotungstic acid.

Aim: Studying the effect of precipitating lipoproteins by Phosphotungstic acid applied to the fraction of exosomes previously isolated by SEC to increase the specific exosome fraction.

Materials and methods: Exosomes are isolated from human plasma by size exclusion chromatography columns (HansaBioMed, Estonia). Lipoproteins are precipitated by 2.35 M Sodium Phosphotungstate and 2.0 M Magnesium Chloride. Supernatant is separated from the pellet by centrifugation and investigated by Western blot using anti-CD9 antibody (ThermoFisher, Darmstadt) as a standard exosomal marker. Levels of LDL and HDL in plasma, eluted SEC fractions and the collected supernatant after phosphotungstic acid precipitation, are measured by using routine Clinical Chemistry methods (Siemens, Eschborn).

Results: Values of LDL and HDL drop by approximately $98 \%$ and $90 \%$ respectively after size exclusion as compared to the original plasma sample. The value of LDL drops again to approximately 50\% after Phosphotungstic acid precipitation of lipoproteins in the exosome containing SEC fraction.

We observed that Phosphotungstic acid, besides precipitating lipoproteins, unfortunately also co-precipitates all the exosomes as well. By serially reducing the molarity of the Phosphotungstic acid used, the LDL level still drops but with different effects on exosomal distribution between supernatant and pellet as seen by Western Blot. This enables us to use the supernatant, now almost free of LDL, in downstream analysis. 


\title{
DGKL-P042
}

\section{Evaluation of cobas $\mathbf{m} 511$ hematology analyzer in routine diagnostic}

\author{
Nico Vogt; Hannsjörg Baum \\ Regionale Kliniken Holding RKH GmbH, Zentrum für Laboratoriumsmedizin, Mikrobiologie, Blutdepot, Ludwigsburg, Germany
}

Objective: A complete blood count is a fast and reliable diagnostic tool. An automated cell counter samples and counts many cells, the results are very precise. However, certain abnormal cells or aggregates in the blood may not be identified correctly, requiring manual review of the instrument's results. Preparation and staining of slides of patient blood samples as well as the microscopic viewing is time consuming. We used the cobas m511 (Roche) with bloodhound technology to automate these processes and compared the results to our haematologic routine analyser Nexus 120 (HORIBA) and the manual leucocyte differentiation.

Methode: Routine samples of complete blood counts and reticulocytes were measured on cobas m511 bloodhound directly following routine measurement and compared to routine analyser results using linear regression and passing-bablock regression with the statistic software "R" including package "mrc". Monocytes, eosinophil and basophil cells were also compared to the manual count.

Result: Comparison of 882 complete blood counts including 126 reticulocyte counts to the routine analyser showed high correlation $\left(\mathrm{R}^{\wedge} 2>0,95\right)$ for WBC, Hb, neutrophils and lymphocytes, a still good correlation $\left(\mathrm{R}^{\wedge} 2>0,90\right)$ for $\mathrm{RBC}$, HCT, MCH and thrombocytes and some correlation $\left(0,7<R^{\wedge} 2<0,90\right)$ for $M C V\left(R^{\wedge} 2=0,88\right)$, monocytes, eosinophiles and reticulocytes $\left(R^{\wedge} 2=0,84\right)$. Correlation was poor $\left(\mathrm{R}^{\wedge} 2<0,7\right)$ for MCHC, RDW, MTV and basophils.

The result of the automated estimation of erythroblasts $(n=51)$ compared to the much more variable manual count showed a nice correlation $\left(\mathrm{R}^{\wedge} 2=0,895\right)$.

Further measurements of monocytes, eosinophils and basophils were compared to the manual routine method for both, the cobas $\mathrm{m} 511$ bloodhound and the routine analyser. For all three, a poor correlation of both analysers to the routine method was detected, with slightly higher correlation for the cobas $\mathrm{m} 511$.

Conclusion: In conclusion, most parameters are similar measured by both systems. Surprisingly, the measurement of erythrocyte and thrombocyte volumes does only in part correlate well, while for example neutrophil and lymphocytecell counts fit much better. These differences in volume and number of erythrocytes are most probably due to the different methods used.

Taken together, the automated workflow of the cobas m511 bloodhound saves a lot of time for special probes that include aggregates or uncommon cells of hemato-oncologic patients. Further, the reduced hands-on time and the faster turn-around time compared to manual staining could be beneficial for samples of the emergency unit.

\section{DGKL-P043}

\section{Induction of Tissue Factor by anti- $\beta 2 \mathrm{GPI}$ antiphospholipid antibodies is mediated by TNF $\alpha$}

\author{
Anne Hollerbach ${ }^{1}$; Nadine Müller-Calleja ${ }^{2}$; Karl Lackner ${ }^{2}$ \\ ${ }^{1}$ Universitätsmedizin Mainz, Centrum für Thrombose und Hämostase / Institut für klinische Chemie und Laboratoriumsmedizin, Mainz, Germany; \\ ${ }^{2}$ Universitätsmedizin Mainz, Institut für klinische Chemie und Laboratoriumsmedizin, Mainz, Germany
}

Background: In the past it has been shown that antiphospholipid antibodies (aPL) induce several proinflammatory and procoagulant cellular responses e.g. in monocytes and endothelial cells, which are believed to contribute to the clinical manifestations of the antiphospholipid syndrome (APS). Tissue factor (TF) has been implicated in the pathogenesis of APS for many years and it has been shown that TF can be induced by aPL. We have recently shown that both anticardiolipin (aCL) and anti- $\beta 2$-glycoprotein I (anti- $\beta 2 \mathrm{GPI}$ ) aPL induce tumor necrosis factor (TNF) $\alpha$ in monocytes. However, the underlying signaling pathways are completely different.

Aims of the study: Analysis if TF induction by different aPL is direct or mediated by proinflammatory cytokines, e.g. TNF $\alpha$.

Materials and Methods: To study this issue we analyzed the ability of three human monoclonal aPL with distinctly different antigenic specificities to induce TF mRNA in monocytes (MM1) and endothelial cells (HUVEC). As inhibitors we used anti-TNF $\alpha$ antibody adalimumab and the endosomal NOX inhibitor niflumic acid (NFA). TF protein expression was measured by flow cytometry and endosomal ROS generation was detected by use of the fluorogenic reagent OxyBURST Green H2HFF-BSA. Results obtained with human monoclonal aPL were validated with IgG-fractions obtained from patients with APS.

Results: A human monoclonal anticardiolipin aPL induced TF mRNA in MM1 and HUVEC cells within $1 \mathrm{~h}$, with a maximum at $3 \mathrm{~h}$. TF-induction was blocked by NFA, whereas adalimumab had no discernable effect. Induction of TF mRNA by aPL reactive against $\beta 2 \mathrm{GPI}$ followed a slower time course and was completely inhibited by adalimumab, NFA had no effect. The inhibitory effect of adalimumab on anti- $\beta 2 \mathrm{GPI}$ antibodies was further confirmed in MM1 cells, as the expression of TF protein and ROS production were also fully blocked by adalimumab. These data could be confirmed with patient IgG, as TF mRNA induction was only reduced by adalimumab if IgG fraction contained anti- $\beta 2 \mathrm{GPI}$ activity. Discussion/Conclusion: The induction of TF by antiphospholipid antibodies is dependent on antigen specificity. While anticardiolipin aPL are able to induce TF transcription directly, the induction of TF by anti- $\beta 2 \mathrm{GPI}$ aPL is mediated by TNF $\alpha$. All observed effects on TF induction by anti- $\beta 2$ GPI aPL were blocked by the TNF $\alpha$ inhibiting antibody adalimumab. 


\title{
DGKL-P044
}

\section{Platelet Biology Revisited}

\author{
Hans-Åke Fabricius \\ privat, Grundlagenforschung, Esgrus, Germany
}

Thrombozyten können Tumorzellproliferation und Metastasierung wesentlich erleichtern. Zirkulierende Tumorzellen akquirieren eine für eine erfolgreiche metastatische Aussaat wesentliche Schicht von Plättchen. Plättchen werden bei Kontakt mit einer Tumorzelle schnell aktiviert und setzen Mediatoren und Substanzen frei, die nicht nur für die Gerinnung, sondern auch für Signalkaskaden der Zellproliferation wichtig sind. In vitro hängt das Wachstum von Zellen generell von der externen Zufuhr von Wachstumsfaktoren ab, die aus dem Serumzusatz im Kulturmedium oder aus anderen solche Faktoren enthaltenden Supplementen stammen. Wenn dieses Supplement weggelassen wird, hören die Zellen auf zu wachsen. Serum enthält Signal-substanzen, die bei der Gerinnung aus Plättchen freigesetzt werden. Wachstumsfaktoren werden in Megakaryozyten synthetisiert und in vivo in $\alpha$-Granula von Thrombozyten transportiert. Danach müssen Plättchen eine wichtige Rolle bei der Kontrolle des Zellwachstums in vivo spielen.

Wir zeigen einen Mechanismus, den eine breite Vielzahl von Zellen, welche in vivo zur Proliferation bestimmt sind nutzen, um Zugang zu Wachstumsfaktoren aus Plättchen zu erhalten.

Unsere Zellkulturexperimente zeigen, dass plättchenfreies Plasma das Zellwachstum nicht fördern kann. Im Gegensatz dazu wachsen Tumorzelllinien, die einem kurzen täglichen Kontakt mit Plättchen ausgesetzt sind, kontinuierlich weiter, ganz ohne Serumzusatz.

Wir zeigen auch, dass permanent proliferierende Tumorzellen in vitro Thrombozyten-Rezeptoren besitzen und dass aktivierte Zellen in vitro solche Rezeptoren beim Eintritt in den Zellzyklus entwickeln und dass primäre Zelllinien in der log-Phase ihres Lebenszyklus solche Rezeptoren exprimieren. Diese Rezeptoren verschwinden allmählich, wenn die primären Zelllinien die logarithmische Phase des Zellwachstums beenden. Plättchen setzen ihren Inhalt in unmittelbarer Nähe zu geeigneten RTKs auf der Zelloberfläche frei. Tumorzellen können auch das andockende Plättchen aufnehmen, welches dann seinen Inhalt direkt in das Zytoplasma der Zelle abgeben kann.

Vorstehende Beobachtungen führen zu der Folgerung, dass

1. Tumorzelllinien in vitro nicht in der Lage sind, Wachstum aufrecht zu erhalten ohne externe Zufuhr von Signalsubstanzen, welche Zellwachstum fördern. Diese Beobachtung widerspricht einem grundlegenden Hallmark of Cancer.

2. Die meisten zur Proliferation bestimmten Zellen besitzen einen Mechanismus, um auf die in vivo Träger dieser Signalsubstanzen zuzugreifen.

3. Thrombozyten spielen eine wesentliche Rolle bei der Kontrolle der Zellproliferation in vivo.

Auch haben wir eine Klasse von Substanzen gefunden, die verhindern können, dass Plättchen den auf proliferierenden Zellen exprimierten Rezeptor ligieren. Diese Beobachtung könnte Hinweise auf eine neue Klasse von Therapeutika geben, die gegen Metastasen gerichtet sind und damit eher auf das Kontrollsystem für Zellwachstum als auf die Tumorzelle selbst abzielen.

\section{DGKL-P045}

\section{A new insight into Trousseau's syndrome: Tumor cell intrinsic prothrombin (F2) drives tumor progression and contributes to a hypercoagulable state}

\author{
Lisa Pruschinski ${ }^{1}$; Ludwig Eder ${ }^{1}$; Shazad Khokhar ${ }^{1}$; Kashif Muhammad ${ }^{1}$; Sergey Tokalov ${ }^{1}$; Sven Danckwardt ${ }^{2}$ \\ ${ }^{1}$ Universitätsmedizin Mainz, Institut für Klinische Chemie und Laboratoriumsmedizin, Mainz, Germany; ${ }^{2}$ Universitätsmedizin Mainz, Institut für \\ klin. Chemie und Laboratoriumsmedizin, Center for Thrombosis and Hemostasis, Mainz, Germany
}

Thrombin (F2) is a key enzyme of the clotting cascade. Beyond its function in hemostasis, F2 has a wide spectrum of functions in other (patho)physiological processes such as embryogenesis, inflammation but also during tumorigenesis. In adults F2 is exclusively expressed in the liver and secreted into the circulation, while ubiquitous (extrahepatic) F2 appears to play an important functional role during embryogenesis.

Here we assessed whether extrahepatic F2 expression is a functionally relevant driver of F2-associated pathologies. Using a newly generated transgenic reporter mouse model („D-Insight“) combined with molecular optical in vivo imaging, we discover extrahepatic F2 expression in sarcomas. We identify that tumor-derived F2 is functionally active; it has direct tumor-promoting properties and contributes to a tumorassociated hypercoagulable state. Due to the high and ubiquitous F2 expression during embryonic development, we speculated that epigenetic regulation might represent an important switch that accounts for extrahepatic F2 expression in tumors. Employing a systematic small molecule compound screening based on D-Insight transgenic cell model systems, we obtain evidence suggesting epigenetic modulation of F2. This finding may represent an explanation for aberrant F2 expression during tumorigenesis and thereby shed novel light onto the association between cancer and excessive procoagulatory activities first noted by Armand Trousseau in 1965. This finding may also pave the way for novel 'targeted' therapeutics in the future. 


\section{DGKL-P046}

\section{Revisiting defects of the contact system of blood coagulation: Detection and differential diagnosis of prekallikrein (PK) deficiency}

Stefanie Sollfrank ${ }^{1}$; Stefano Barco ${ }^{2}$; Alice Trinchero ${ }^{2}$; Luigi Tomao ${ }^{3}$; Barbara Zieger ${ }^{4}$; Johanna A. Kremer Hovinga ${ }^{5}$ : Laura Conti ${ }^{6}$; Anke

Adenäuer'; Erwin Miloni'; Karl Lackner'; Heidi Rossmann'; Bernhard Lämmle ${ }^{2}$

${ }^{1}$ Universitätsmedizin Mainz, Institut für Klinische Chemie und Laboratoriumsmedizin, Mainz, Germany;

${ }^{2}$ Universitätsmedizin Mainz, Centrum für Thrombose und Hämostase (CTH), Mainz, Germany;

${ }^{3}$ IRCCS Bambino Gesù Children's Hospital, Rome, Department of Pediatric Hematology/Oncology, Rome, Italy;

${ }^{4}$ Universitätsklinikum Freiburg i. Br., Zentrum für Kinder- und Jugendmedizin, Klinik IV, Freiburg i. Br., Germany;

Inselspital Universitätsspital Bern, Universitätsklinik für Hämatologie und Hämatologisches Zentrallabor, Bern, Switzerland;

${ }^{6}$ Istituto Nazionale Tumori “Regina Elena”, Roma, UOSD Patologia Clinica, Rome, Italy;

${ }^{7}$ Practicing Physician, Bern, Bern, Switzerland

Objectives: PK and high-molecular-weight kininogen (HK) deficiencies are ultra-rare, autosomal-recessive defects of the contact system caused by mutations in the KLKB1 and KNG1 genes, respectively. Since carriers do not manifest a bleeding phenotype, a correct diagnosis is essential to prevent the administration of prohemostatic agents or plasma, and to avoid delay of surgery.

Aims: We describe a new case of PK deficiency identified at UMC Mainz. In addition, we performed a systematic review of the literature in order to i) collect blood material for genetic studies of reported PK deficient cases and ii) provide an individual patient analysis for clinical associations and diagnostic criteria (ongoing).

Methods: MEDLINE and EMBASE were searched without time and language restrictions. Reference lists of retrieved articles, abstract books of hematology congresses and grey literature were manually reviewed. Authors of articles on PK deficient cases not assessed for genetic defects, were contacted in order to retrieve blood samples. PK:C and HK:C were measured using PK and HK deficient plasmas, respectively. PK and HK antigens (PK:Ag and HK:Ag) were determined by ELISA. Routine aPTT and coagulation factor measurements were performed using an ACL TOP (IL). Genetic testing was performed by Sanger-, Pyrosequencing and/or NGS.

Results: Our patient was a 69-year-old woman of African descent referred for preoperative evaluation of an isolated aPTT prolongation. Diagnosis of PK deficiency was based on absent PK:C and PK:Ag ( $\leq 1 \%$ of normal, each). The homozygous KLKB1 mutation p.Ser151Phefs 34 was found, which was not yet described as a cause of PK deficiency, but had been detected in the African sub-collective (MAF 1.3\%) of the 1000 Genomes cohort.

A total of 1,913 studies were identified by systematic literature review. Eleven studies with genetic data were found. Blood from 4 unrelated European families without previous genetic testing was analysed, including 3 index cases and 5 relatives. The KLKB1 mutation p.Cys548Tyr was found in 2 families with one index patient being homozygous. This data and the literature suggest that p.Cys548Tyr may be the most frequent KLKB1 mutation in Caucasians, associated with lacking PK:C but low amount of PK:Ag.

One patient diagnosed with PK deficiency based on (incomplete) normalization of aPTT with increased preincubation time and low PK:C (7\%) was found to carry compound heterozygous mutations in KNG1 (c.1038+1G > A and c.1165C > T, p.Arg389*) but no mutations in KLKB1.

Conclusions. PK deficiency may not be as rare as previously thought, especially in subjects of African origin. Incomplete normalization of severely prolonged aPTT upon prolonged preincubation and low PK levels are not sufficient for the diagnosis of PK deficiency. Our latter case and data from literature suggest that patients with HK deficiency usually present with moderately low PK levels: therefore, PK and HK:C and HK:Ag should be determined.

\section{DGKL-P047}

\section{Functional and genetic characterization of patients with Glanzmann Thrombasthenia}

Katharina von Bargen'; Max-Joseph Kraus²; Erwin F. Strasser ${ }^{3}$; Günther Kappert ${ }^{4}$; Susan Halimeh ${ }^{4}$; Franziska Knüttgen ${ }^{1}$; Tobias Flieder'; Cornelius Knabbe ${ }^{1}$; Ingvild Birschmann ${ }^{1}$

${ }^{1}$ Herz- und Diabeteszentrum Nordrhein-Westfalen, Institut für Laboratoriums- und Transfusionsmedizin, Bad Oeynhausen, Germany;

${ }^{2}$ Praxis Geiselgasteig, Praxis Geiselgasteig, Grünwald, Germany;

${ }^{3}$ Universitätsklinikum Erlangen, Transfusionsmedizinische und Hämostaseologische Abteilung, Erlangen, Germany;

${ }^{4}$ Gerinnungszentrum Rhein-Ruhr, Gerinnungszentrum Rhein-Ruhr, Duisburg, Germany

Objective: Platelets play a central role in hemostasis, a physiological process which stops bleeding. A crucial component of hemostasis is the fibrinogen receptor on the surface of the platelets. It is an integrin which consists of two subunits, the $\alpha$ IIb- and beta3-subunit. The receptor is necessary to cross-link platelets during aggregation. Quantitative or qualitative abnormalities of the integrin $\alpha \operatorname{IIb} \beta 3$ (GPIIb/IIIa) lead to the development of Glanzmann Thrombasthenia (GT). The defects of the platelet integrin $\alpha \operatorname{Ilb} \beta 3$ resulting from pathogenic mutations in either the ITGA2B ( $\alpha \mathrm{IIb})$ or the ITGB3 ( $\beta 3$ ) gene on chromosome 17q21.31-32. GT is a platelet dysfunction, which is characterized by spontaneous mucosal bleeding caused by the absence of aggregation. 
Usually routine diagnostics and the functional analysis by light transmission aggregometry are sufficient for the distinct diagnosis. In addition, flow cytometry as well as DNA sequencing can be performed for more detailed analysis.

Methods: In our study we analyzed six patients with GT. For the functional analysis we used the PFA-200 system and the light transmission aggregometry. The genetic characterization was carried out by sequencing the respective exon region with the Sanger method. Additionally we studied the surface expression of $\alpha \operatorname{IIb} \beta 3$ by flow cytometry.

Results: All patients in our GT population show pathological PFA-200 closure times as well as an absence of platelet aggregation in response to all physiological agonists. Only with Ristocetin is a reversible aggregation detectable. The surface expression analysis by flow cytometry displays an absence of the fibrinogen receptor in patient 3, 4, 5 and 6. Only patient 1 and 2 have an expression about 5-15\% compared to a healthy donor population.

The genetic characterization showed a homozygote $\mathrm{C}$ to $\mathrm{G}$ mutation in the exon 18 of the IGTA2B gene in patient 5 and 6 (pair of siblings). This already described pathogenic mutation is an exchange of glutamine to histidine. In another sibling pair (patient 1+2), a mutation in exon 10 of ITGB3 gene was identified. It is a novel mutation which resulted in a cysteine to tryptophan amino acid substitution. Furthermore, two other novel mutations in the ITGA2B gene could be identified in patient 3 and 4 . The patient 3 showed a $\mathrm{T}$ to $\mathrm{A}$ transition which leads to an amino acid substitution of valine to glutamic acid on position 422. Whereas patient 4 exhibits a frameshift mutation caused by an 8 bp deletion in exon 14 . Conclusions: All six patients show pathological PFA-200 closure times and the for GT typical pathognomonic aggregation. The flow cytometry analysis displays the quantitative defect of the fibrinogen receptor in all patients. Additionally three novel GT mutations could be identified in our study, which are discussed in our scientific contribution. We suggest that the GT phenotype is the result of the mutations outlined above.

\section{DGKL-P048}

\section{A case study: Is core-fucosylated prostate-specific antigen (PSA) a refined biomarker for differentiation of benign prostate hyperplasia (BPH) and prostate cancer of different aggressiveness?}

Robert Lang'; Michael Vogeser ${ }^{2}$

${ }^{1}$ Roche Diagnostics, Penzberg, Germany; ${ }^{2}$ Hospital of the Ludwig-Maximilians University, Institute of Laboratory Medicine, Munich, Germany

Objectives: Prostate cancer (PCa) represents a major cause for cancer death in men worldwide. Novel non-invasive methods are still required for differentiation of non-aggressive from aggressive tumors. Recently, changes in PSA glycosylation pattern, such as core-fucosylation have been described in PCa. The objective of this study was to evaluate whether the core-fucosylation determinant of serum PSA may serve as refined marker for differentiation between benign prostate hyperplasia (BPH) and PCa or identification of aggressive PCa.

Method: A previously developed LC-MS/MS-based strategy was used for multiplex analysis of core-fucosylated PSA (fuc-PSA) and total PSA levels in sera from $50 \mathrm{BPH}$ and $100 \mathrm{PCa}$ patients of different aggressiveness (Gleason scores 5-10) covering the critical grey area (2-10 ng/ml). Sample preparation comprised an immunoaffinity capture step to enrich total PSA from human serum using anti-PSA antibody coated magnetic beads followed by consecutive two-step on-bead partial deglycosylation with endoglycosidase F3 and tryptic digestion prior to LC-MS/ MS analysis.

Results: Our data showed that the ratio fuc-PSA to total PSA (\%-fuc-PSA) was decreased in aggressive PCa (Gleason score > 6) in comparison to non-aggressive PCa (Gleason score $\leq 6$ ) and yielded a 5 to $8 \%$ increase in the area under the curve (AUC $=0.60$ ) than the currently used total PSA (AUC $=0.52)$ and \%-free PSA (AUC $=0.55)$ tests. In contrast, both fuc-PSA and \%-fuc-PSA had no diagnostic value for differentiation of BPH from PCa.

Conclusions: A higher tendency for differentiation of non-aggressive and aggressive PCa was obtained using \% fuc-PSA compared to conventional diagnostic PCa markers. Therefore, \%-fuc-PSA should be further investigated e.g. in larger patient cohorts or by more precise methods whether it could be clinically used in PCa diagnosis.

\section{Posterwalk Onkologie, Hämatologie und Hämostaseologie II}

\section{DGKL-P049 / PV07}

\section{Large-scale identification of functional miRNA targeting reveals cooperative regulation of the hemostatic network}

Jamie Nourse' ${ }^{1}$ Juliane Braun²; Karl Lackner'; Stefan Hüttelmaier²; Sven Danckwardt ${ }^{3}$

${ }^{1}$ University Medical Center of the Johannes Gutenberg University, Institute for Clinical Chemistry and Laboratory Medicine, Mainz, Germany; ${ }^{2}$ Martin Luther University Halle (Saale), Institute of Molecular Medicine, Halle, Germany; ${ }^{3}$ University Medical Center of the Johannes Gutenberg University, Center for Thrombosis and Hemostasis and Institute for Clinical Chemistry and Laboratory Medicine, Mainz, Germany

miRNAs confer robustness to complex molecular networks regulating biological functions. However, despite the involvement of miRNAs in almost all biological processes, and the importance of the hemostatic system for a multitude of actions in and beyond blood coagulation, the 
role of miRNAs in hemostasis is poorly defined. Here we comprehensively illuminate miRNA-mediated regulation of the hemostatic system in an unbiased manner. In contrast to widely applied association studies, we used an integrative screening approach that combines functional aspects of miRNA silencing with biophysical miRNA interaction based on RNA pull-downs (miTRAP) coupled to next generation sequencing. Examination of a panel of 27 hemostatic-associated gene 3'UTRs revealed the majority to possess substantial Dicer-dependent silencing capability, suggesting functional miRNA-targeting. miTRAP revealed 150 specific miRNA interactions with 14 3'UTRs, of which 52, involving 40 miRNAs, were functionally confirmed. This includes cooperative miRNA regulation of key hemostatic genes comprising pro- (F7, F8, F11, FGA, FGG, KLKB1) and anti-coagulant (SERPINA10, PROZ, SERPIND1, SERPINC1) as well as fibrinolytic (PLG) components. Bioinformatic analysis of miRNA functionality reveals established and potential novel links between the hemostatic system and other pathologies, such as cancer, bone metabolism and renal function. Our findings provide, along with an in-vivo proof of concept, deep insights into the regulatory network of miRNAs regulating the hemostatic system and present a foundation for novel targeted therapeutics for correction of de-regulated hemostasis and associated processes in the future.

\section{DGKL-P050}

\section{Large-scale identification of functional miRNA targeting reveals cooperative regulation of the hemostatic network}

James Nourse ${ }^{1}$; Juliane Braun²; Karl Lackner'; Stefan Hüttelmaier²; Sven Danckwardt ${ }^{3}$

${ }^{1}$ Universitätsmedizin Mainz, Institut für Klinische Chemie und Laboratoriumsmedizin, Mainz, Germany; ${ }^{2}$ Martin Luther University Halle (Saale), Institute of Molecular Medicine, Halle, Germany; ${ }^{3}$ Universitätsmedizin Mainz, Institut für klin. Chemie und Laboratoriumsmedizin, Center for Thrombosis and Hemostasis, Mainz, Germany

miRNAs confer robustness to complex molecular networks regulating biological functions. However, despite the involvement of miRNAs in almost all biological processes, and the importance of the hemostatic system for a multitude of actions in and beyond blood coagulation, the role of miRNAs in hemostasis is poorly defined. Here we comprehensively illuminate miRNA-mediated regulation of the hemostatic system in an unbiased manner. In contrast to widely applied association studies, we used an integrative screening approach that combines functional aspects of miRNA silencing with biophysical miRNA interaction based on RNA pull-downs (miTRAP) coupled to next generation sequencing. Examination of a panel of 27 hemostatic-associated gene 3'UTRs revealed the majority to possess substantial Dicer-dependent silencing capability, suggesting functional miRNA-targeting. miTRAP revealed 150 specific miRNA interactions with 14 3'UTRs, of which 52, involving 40 miRNAs, were functionally confirmed. This includes cooperative miRNA regulation of key hemostatic genes comprising pro- (F7, F8, F11, FGA, FGG, KLKB1) and anti-coagulant (SERPINA10, PROZ, SERPIND1, SERPINC1) as well as fibrinolytic (PLG) components. Bioinformatic analysis of miRNA functionality reveals established and potential novel links between the hemostatic system and other pathologies, such as cancer, bone metabolism and renal function. Our findings provide, along with an in-vivo proof of concept, deep insights into the regulatory network of miRNAs regulating the hemostatic system and present a foundation for novel targeted therapeutics for correction of de-regulated hemostasis and associated processes in the future.

\section{DGKL-P051}

\section{Platelet count in autoimmunthrombocytopenia during pregnancy}

Theresa Wagner'; Günther Giers'; Folker Wenzel ${ }^{2}$

${ }^{1}$ Universität Düsseldorf, Institut für Transfusionsmedizin, Düsseldorf, Germany; ${ }^{2}$ Hochschule Furtwangen, Medical and Life Sciences, Villingen Schwenningen, Germany

Introduction: During pregnancy thrombocytopenias can be observed in up to $15 \%$ of the women caused by different entities. In one of these entities pregnant women suffering from an autoimmunthrombocytopenia (AITP). The maternal autoantibodies led not only to a thrombocytopenia in the mother but also in the fetus. To minimize bleeding risks there are different therapeutic options. In this study we examined the therapeutic success for mother and fetus.

Material and Mehods: 16 pregnant women suffering from AITP could be observed. Different maternal blood markers (e.g. platelet count, reticulocytes, LDH) were monitored, the fetus was sonographically examined. The women were treated by anti-Rh0-antibodies (1.200 mikrog) in the 30. - 32. week of gestation, and fetal platelet count were determind in the 34. - 35. week of gestation.

Results: In the 16 patients no adverse therapeutic effects could be observed. IN the pregnant women, platelet count showed a significant $(\mathrm{p}<0,05)$ increase from values $<20.000 / \mathrm{mikrol}$ to values above $60.000 / \mathrm{mikrol}$. In the fetuses, 5 cases $(31,3 \%)$ showed platelet counts above $100.000 / \mu \mathrm{l}$ and could be delivered via naturalis. Overall, no fetal anemia or hemolysis could be observed.

Conclusion: In maternal AITP, anti-Rh0-immunoglobulin treatment seems to be a possible therapeutic option to increase maternal platelet count without fetal hemolysis. 


\section{DGKL-P052}

\section{Hematopoietic stem cells can differentiate into restricted myeloid progenitors before cell division in mice}

Ben Wielockx

IKL - Dresden, Dresden, Germany

Hematopoietic stem cells (HSCs) continuously replenish all blood cell types through a series of differentiation steps and repeated cell divisions that involve the generation of lineage-committed progenitors. However, whether cell division in HSCs precedes differentiation is unclear. To this end, we used an HSC cell tracing approach and Ki67RFP knock-in mice, in a non-conditioned transplantation model, to assess divisional history, cell cycle progression, and differentiation of adult HSCs. Our results reveal that HSCs are able to differentiate into restricted progenitors, especially common myeloid, megakaryocyte-erythroid and pre-megakaryocyte progenitors, without undergoing cell division and even before entering the S phase of the cell cycle. Additionally, the phenotype of the undivided but differentiated progenitors correlated with expression of lineage-specific genes and loss of multipotency. Thus, HSC fate decisions can be uncoupled from physical cell division. These results facilitate a better understanding of the mechanisms that control fate decisions in hematopoietic cells.

\section{DGKL-P053}

\section{Quantification of free hemoglobin in plasma: a specrophotometric algorithm}

Michael Paal'; Alexander Lang'2 ; Hennig Georg'; Marie-Luise Buchholtz'; Ronald Sroka²; Michael Vogeser ${ }^{1}$ ${ }^{1}$ Klinikum der Universität München - LMU München, Institut für Laboratoriumsmedizin, München, Germany; ${ }^{2}$ Klinikum der Universität München - LMU München, Laser-Forschungslabor, München, Germany

Background: Assessment of hemolysis is becoming increasingly relevant in critical care. Currently used spectrophotometric methods for the quantification of free hemoglobin (fHB) produce unsatisfactory results in case of hyperbilirubinemia, a frequent condition in patients at risk for intravascular hemolysis. The objective of the current study was therefore to develop a highly selective spectrophotometric fHb quantification method using computational processing.

Methods: The novel second-derivative fitting algorithm uses spectrophotometric data from 350 to $650 \mathrm{~nm}$ recorded with standard instruments as input. For evaluation, plasmas of patients and non-icteric plasma of healthy volunteers were spiked with fHb concentrations up to $200 \mathrm{mg} /$ $\mathrm{dl}$, and compared to methods described in the literature by Harboe, Noe and Fairbanks. All measurements were done in compliance with the bioanalytical method validation protocol from the European Medicines Agency and "Milan consensus" for analytical performance.

Results: Both the second-derivative fitting algorithm as well as the methods of Harboe, Noe and Fairbanks quantified fHb accurately in non-icteric samples, with inaccuracy and imprecision below $10 \%$. For icteric specimen, false high results were obtained with the established formulas for $\mathrm{fHb}$ concentrations below $700 \mathrm{mg} / \mathrm{L}$. In contrast, no interference was found with the second-derivate fitting method for bilirubin concentrations up to $27.2 \mathrm{mg} / \mathrm{dl}$. The lower limits of quantifications for the second-derivative fitting algorithm were specified in agreement with the EMA guideline with $25 \mathrm{mg} / \mathrm{L} \mathrm{fHb}$ for both non-icteric and icteric specimens. The method was shown to be highly selective for quantification of free hemoglobin.

Conclusions: We report a user-friendly computer-based algorithm that allows the accurate quantification of plasma free hemoglobin concentrations by UV/VIS-spectrophotometry in the presence of high bilirubin concentrations. The second-derivative fitting algorithm also quantifies total bilirubin and is therefore named HEBIFit ("Hemoglobin Bilirubin Fit"). Using a uniform sample preparation with a single dilution step HEBIFit can be readily implemented in any laboratory on standard spectrophotometers using a provided Microsoft Excel macro.

\section{DGKL-P054}

\section{Comparison of the heavy/light chain assay to other quantitative methods in the diagnosis of IgM multiple myeloma patients}

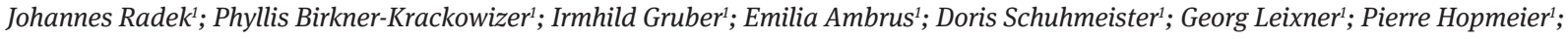
Alexander Claus Haushofer'; Doris Trubert-Exinger ${ }^{1}$

${ }^{1}$ University Hospital St. Pölten, Austria, Institute of Laboratory Medicine, St. Pölten, Austria; ${ }^{2}$ Klinikum Wels-Grieskirchen, Austria, Institute of Medical and Chemical Laboratory Diagnostics with Blood Bank, Wels-Grieskirchen, Austria

Background: The heavy / light chain (HLC) immunoassays (Hevylite ${ }^{\circledR}$, The Binding Site, UK) separately identify and quantify the immunoglobulin isotypes like IgM-Kappa and IgM-Lambda. This enables the calculation of the heavy / light chain Kappa/Lambda ratio (HLCR) which 
provides additional information about the underlying disease. The HLC assays are used for diagnosis, monitoring, response assessment and prognosis of monoclonal gammopathies.

Objective of the study: The aim of the study was to assess the correlation between HLCR and other standard laboratory methods. Results were compared retrospectively with serum immunofixation electrophoresis (sIFE), total IgM, total light chains and serum free light chains (sFLC).

Patients and Methods: Sera from 42 IgM myeloma patients, selected on the basis of positive sIFE results, were studied. sFLC (Freelite ${ }^{\circledR}$, The Binding Site, UK) and HLC were quantified using polyclonal antisera assays on the BNTMII nephelometer (Siemens Healthcare Diagnostics Inc). Total IgM and total light chains were measured on the BNTMII. sIFE was performed using the Sebia Hydrasys 2 system.

Results: An abnormal IgM-HLCR was present in $85,7 \%$ of the IgM samples with a positive sIFE result. Total IgM was abnormal elevated in 76,2 $\%$ and total light chain ratio was abnormal in $47,6 \%$ of the cases.

An abnormal sFLC ratio was found in $100 \%$ of the sIFE positive samples.

Two patients with a biclone (IgM-Lambda, IgG-Kappa) which presented with normal values of total IgM and normal total light chain ratio but abnormal HLCR had been identified.

Conclusion: A 85,7\% and a $100 \%$ agreement with positive sIFE was found with an abnormal IgM-HLCR as well with an abnormal FLC ratio. HLCR was more sensitive than total IgM or total light chain ratio. The separate analysis of involved and uninvolved HLC could give additional information in case of biclonality.

\section{DGKL-P055}

\section{Magnesium Sulfate an alternative in vitro anticoagulant for measuring platelet activation}

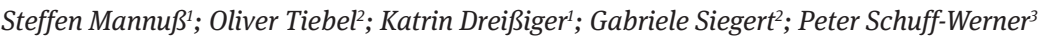

${ }^{1}$ Universitätsmedizin Rostock, Institut für Klinische Chemie und Laboratoriumsmedizin, Rostock, Germany; ${ }^{2}$ Universitätsklinikum Carl Gustav Carus, TU Dresden, Institut für Klinische Chemie und Laboratoriumsmedizin, Dresden; ${ }^{3}$ MDI Laboratorien GmbH Medizinisches Versorgungszentrum, Klinische Chemie, Berlin, Germany

Objective: Platelet activation triggers the expression of CD62p (p-selectin) and CD63 (granulophysin). For measuring activation markers by flow cytometry, citrate anticoagulation is recommended because citrate causes less spontaneous platelet activation than EDTA (Macey 1999, Mody 1999). Beside the measurement of platelet activation markers, the formation of platelet-leukocyte-complexes can be utilized as indicator of platelet activation (Pearson 2009, Nagasawa 2013). In order to classify the impact of the magnesium sulfate anticoagulation on the expression of CD62p, CD63 and the formation of these aggregates, we conducted this investigation. Methods: Whole blood of healthy volunteers was carefully drawn from the cubital vein after release of the tourniquet using a 21-gauge butterfly needle. Blood was collected in commercial tubes anticoagulated either with EDTA, sodium citrate or magnesium sulfate. CD62p and CD63 expression was determined by flow cytometry (Navios, Beckman Coulter), whereas platelet-leukocyte-aggregates measurement was performed using a flow cytometer from BD Bioscience (FACS Canto II). Statistical analysis and graphical presentation of the results was done by SigmaPlot software version 11 .

Results: In EDTA anticoagulated blood we could measure a spontaneous CD62p and CD63 expression, whereas such an activation was not observed in citrate and magnesium sulfate samples. On the other hand the increase of activation after adding agonists (ADP, arachidonic acid) is between 20 and $49 \%$; after 300 minutes the activation is 2-3 fold higher. Another parameter of platelet activation is the proportion of leukocytes binding activated platelets (CD42b positive granulocytes, lymphocytes and monocytes) as shown in the table.

\begin{tabular}{lrcr}
\hline & & \multicolumn{2}{c}{ Percentage of CD42b-positive cells } \\
\cline { 2 - 4 } & Granulocytes & Lymphocytes & Monocytes \\
\hline EDTA & $21,63+/-9,11 \%$ & $14,53+/-7,62 \%$ & $26,82+/-15,82 \%$ \\
Citrate & $54,09+/-20,89 \%$ & $14,97+/-8,09 \%$ & $71,31+/-18,61 \%$ \\
MgSO4 & $44,92+/-16,12 \%$ & $14,53+/-7,30 \%$ & $77,77+/-16,74 \%$ \\
\hline
\end{tabular}

Conclusions: The results of this study clearly show that magnesium sulfate is a suitable anticoagulant for the measurement of platelet activation markers: In contrast to EDTA, magnesium sulfate anticoagulated platelets show no activation although activation by agonists is still working. Magnesium sulfate is therefore an alternative to the citrate anticoagulation for measuring platelet activation. 


\section{DGKL-P056}

\section{Identification of SNPs as independent risk factors in patients with miscarriage and deep vein thrombosis}

Maximilian Kittel ${ }^{1}$; Parviz Ahmad-Nejad²; Alexandra Kritter ${ }^{3}$; Michael Neumaier ${ }^{3}$; Verena Haselmann ${ }^{3}$

${ }^{1}$ Universitätsmedizin Mannheim, Institut für klinische Chemie, Mannheim, Germany; ${ }^{2}$ HELIOS Klinikum Wuppertal, Witten/Herdecke University, Institute for Medical Laboratory Diagnostics, Center for Clinical and Translational Research, Wuppertal,Germany; ${ }^{3}$ Medical Faculty Mannheim of Heidelberg University, Institute for Clinical Chemistry, Mannheim, Germany

Background: Thrombophilia leads to an increased risk for developing thromboembolic events like deep vein thrombosis or a pulmonary embolism, and is additionally associated with abortion or reduced fertility. According to ACCP guidelines, only factor V Leiden and prothrombin G20210A mutation are being concerned in respect to therapeutic decisions. As reanalysis of the Leiden collective has identified additional sequence variations as risk factors for thrombophilia, we wanted to confirm these variants in an independent case-control study.

Material and Methods: Patients with abortion, thromboembolic events or a positive family history were recruited at our hemostaseology outpatient department within the last years. As controls, age and sex matched gDNA samples from anonymized blood donors were used.

For each patient and control sample gDNA from 500 $\mu$ EDTA blood was isolated and following sequence variations were analyzed by pyrosequencing: CYP4V2 (rs13146272), F2 (rs1799963), F5 (rs6025; rs4524; rs1800595), F9 (rs6048), F12 (rs1801020), FGB (rs1800790), GP6 (rs1613662), KLKB1 (rs3087505), NR1I2 (rs1523127), RGS7 (rs670659), SERPINC1 (rs2227589), and SERPINE1 (rs587776796). A student t-test was performed for statistical differences between case- and control group. Logistic regression models were used to calculate the odds ratio, 95\% confidence interval. Results: F2 (rs1799963), F5 (rs6025; rs4524; rs1800595), GP6 (rs1613662) and NR1I2 (rs1523127) were identified as independent risk factor for developing a thromboembolic event in our study population. F2 (rs1799963), CYP4V2 (rs13146272), F9 (rs6048), F12 (rs1801020), and FGB (rs1800790) have shown to be associated with an increased risk of miscarriage, and CYP4V2 (rs13146272), F5 (rs6025; rs4524; rs1800595), GP6 (rs1613662), SERPINC1 (rs2227589) are associated with a positive family history for deep vein thrombosis. Interestingly, in the group of patients with abortion, Prothrombin G20210A, F12C46T and CYP4V2 proved to have the highest Odds ratios.

Conclusion: The study's results support the hypothesis that certain single-nucleotide variations are strongly correlated with the risk of thromboembolic events and miscarriage. According to our data, further sequence variations should be considered in respect to therapeutic decisions. However, further randomized clinical trials are warranted in order to prove the clinical relevance and for assessment of the cumulative risk.

\section{Posterwalk Inflammation, Infektion und Autoimmunerkrankungen I}

\section{DGKL-P057 / PV08}

\section{High- throughput Single Cell RNA Sequencing reveals monocyte subpopulations expressing variable immune Receptors}

Eileen Reinz ${ }^{1}$; Carsten Sticht ${ }^{2}$; Michael Neumaier ${ }^{1}$; Tina Fuchs ${ }^{3}$

${ }^{1}$ Medizinische Fakultät Mannheim, Universität Heidelberg, Institut für Klinische Chemie, Mannheim, Germany; ${ }^{2}$ Medizinische Fakultät Mannheim, Universität Heidelberg, Zentrum für Medizinische Forschung, Mannheim, Germany; ${ }^{3}$ Universitätsklinikum Mannheim, Institut für Klinische Chemie, Mannheim, Germany

Background: Recent evidence demonstrates the expression of immunoglobulin and T cell receptor-based combinatorial immune receptors outside the cognate immune system in monocytes/macrophages of healthy individuals and patients with pathological conditions.

Objectives: High-throughput single cell sequencing makes it feasible to demonstrate and characterize the expression of the variable immune receptors in monocytes in an unprecedented cell-specific manner and quantitative fashion.

Material and methods: A microdroplet-based scRNA-seq dataset from 7.500 PBMCs of a healthy individual provided by 10xGenomicsTM was evaluated for the existence of myeloid subpopulations expressing variable immune receptors. Additionally, we performed parallel highthroughput analysis of single cell 5' gene expression and immune receptor profiling on 10.000 monocytes and 7.000 B cells using the same microdroplet-based scRNA-seq platform from 10x GenomicsTM.

Results: The 10xGenomicsTM PBMC dataset revealed two small subpopulations of monocytes expressing immunoglobulins and T cell receptors, respectively. These subpopulations showed distinct transcriptomic features and expression of monocyte-specific Ig and TCR clonotypes, respectively. These initial results could be confirmed by our own high-throughput sequencing approach. Prevalence of these cells is in accord with our previous observation of immunoreceptor-positive myeloid cells.

Discussion: Our results demonstrate that the novel technique of microdroplet-based single cell transcriptome sequencing is a powerful tool to detect minor but distinct subpopulations in a heterogeneous environment (PBMC). The parallel analysis of single cell transcriptomes and immune receptor profiling leads to a detailed characterization of single cells and the full-length sequences of the complete immune receptor clonotype (antibody or T cell receptor) at the same time. Using this sensitive high-throughput approach we uncovered a remarkable 
complexity of two monocyte-based variable immune systems that until now could not be characterized in such detail and certainty. This highthroughput single cell technique will broaden the spectrum of experimental approaches and will give new insights into the properties of these novel monocyte-based variable immune receptors on the single-cell level in health and disease.

\title{
DGKL-P058 / PV09
}

\section{The relation of uremia-induced microglial activation via KCa3.1 and the p38-MAPK-pathway and inflammasome- mediated neuronal dysfunction}

\author{
Silke Zimmermann ${ }^{1}$; Akash Mathew²; Moritz Ahrens' ${ }^{2}$ Ludwig Scheckenbach²; Berend Isermann ${ }^{2}$ \\ ${ }^{1}$ Universitätsklinikum Magdeburg, Institut für Klinische Chemie und Pathobiochemie, Magdeburg, Germany; ${ }^{2}$ Universitätsklinikum Magdeburg, \\ IKCP, 39120 Magdeburg, Germany
}

Background: Chronic kidney disease (CKD) causes cognitive impairment. Uremic toxins affect neuronal function. Molecular mechanisms remain unknown. We hypothesize that uremic toxins cause $\mathrm{K}+$-dyshomeostasis, which contribute to inflammasome activation within the CNS. Aim: We aim to decipher the relevance of $\mathrm{K}+$-dyshomeostasis and the mechanisms of activation in microglia in the CNS leading to sterile inflammation in neurons. We intend to explore the temporal dynamics of $\mathrm{K}+$-dyshomeostasis and inflammasome activation in cerebral cells. Methods: We used thallium-autometallography (Tl-AMG) to monitor cellular $\mathrm{K}+$-metabolism. The potassium analogue thallium is taken up simultaneously with $\mathrm{K}+$ via the same mechanisms from the extracellular space and can be fixed in situ. CKD in mice was induced by 5/6 nephrectomy (5/6 NX). To study cell-activation in the CNS in vivo we conducted two-photon intravital microscopy. We used transgenic mice expressing EGFP under the control of the CX3C chemokine receptor 1 (CXC3R1) promoter (labeling microglia). NLRP3 and Casp-1 knock-out mice (NLRP3-/-, Casp-1-/-, C57BL/6 background) were used to determine the relevance of inflammasome activation. We generated conditional knock out mice for IL-1R and caspase-1 (IL-1RLoxP and caspase-1LoxP), which were crossed with Emx1Cre (neuronal Cre-expression) and Cx3CCre (microglia Cre-expression) mice. We used the specific KCa3.1- inhibitor TRAM-34 to examine its role in microglia cells while being treated with uremic plasma in vitro and in vivo in 5/6 nephrectomized mice. Results: In CKD mice K+-metabolism in the CNS is altered leading to increased expression of Casp-1 and IL-1ß. Two-photon microscopy revealed activation of microglia in mice with CKD. Analyses of wild type C57BL/6 and NX NLRP3-/- mice six weeks after inducing CKD revealed a comparable number of thallium positive activated microglia, indicating that the uremia-induced reactive shift and $\mathrm{K}+$-dyshomeostasis in microglia is a process independent from NLRP3 inflammasome activation. However, in 5/6 NX NLRP3-/- mice as well as in CASP-1-/- mice the decrease of neuronal thallium uptake in somatosensoric cortex was abolished, indicating that the NLRP3 inflammasome is indeed involved in CKD-induced K+-dyshomeostasis of neurons, hence neuronal dysfunction. These current investigations in cell-specific knock-out mice models will be presented. In vitro we show a decreased phosphorylation of p38 after TRAM-34 treatment of microglia. The p38 pathway inhibits the degradation of pro-IL1ß mRNA and proinflammatory mediators causing increase of pro-IL1ß. We further show the activation of microglial cells in CKD leads to secretion of Il1ß, which - unlike in neurons - is independent of cleavage by Casp-1. Conclusion: These data suggest microglial K+ - dyshomeostasis to be an initial step leading to neuronal dysfunction. We propose that activation of microglia is pivotal in the pathophysiology of CKD-induced impairment of brain function.

\section{DGKL-P059 / PV10}

\section{CKD-related uremia-induced disruption of the blood brain barrier and its impact on cognitive decline}

Moritz Ahrens'; ${ }^{1}$ Silke Zimmermann²; Akash Mathew²; Berend Isermann ${ }^{2}$

${ }^{1}$ Universitätsklinikum Magdeburg, Institut für Klinische Chemie und Pathobiochemie, Magdeburg, Germany; ${ }^{2}$ Universitätsklinikum Magdeburg, IKCP, 39120 Magdeburg, Germany

Background: In independent studies we have shown that chronic kidney disease (CKD) causes sterile inflammation in brain cells and hence neuronal dysfunction. We hypothesize that these effects are caused by uremic toxins that are accumulating in the blood of CKD patients. The blood brain barrier (BBB) constitutes a highly selective barrier between blood and brain tissue. Considering our current insights into the mechanistic relevance of neuroinflammation for the cognitive impairment in CKD, the questions whether and if so, how the BBB gets leaky in CKD are of functional relevance.

Aim: Using an in vitro barrier model we intend to scrutinize whether uremic toxins can increase the permeability of the BBB. Further, we want to investigate the effect of uremic plasma on vascular endothelial brain cells. Mechanistically, we intend to study the relevance of the inflammasome and tight junction proteins. Finally, we want to investigate a possible impact of the coagulation protease activated protein $\mathrm{C}$ (aPC) on the integrity of the BBB in CKD.

Methods and materials: We use the immortalized mouse brain endothelial cell line b.End3. To study the barrier function a dextran permeability assay was used. $04 \times 105 \mathrm{~b}$.End3 cells were seeded on $2 \%$ gelatin-coated 24 well cell culture inserts with a pore size of $0.4 \mu \mathrm{m}$ and cultivated for 4-5 days. Once confluent, the cells were treated with either plasma from CKD patients or healthy controls. 4.4 kDa TRITC-conjugated 
dextran was added. Every 2 hours, the inserts were transferred to a new well containing fresh media and he fluorescence intensity was measured. To explore the role of aPC, cells were pretreated for one hour with 20nM aPC. B.End3 cells were stimulated with LPS or uremic plasma. Immunoblotting was used to analyze inflammasome activation.

Results: Significant leakage of dextran was observed following exposure to uremic plasma. Treatment with aPC was partially protective. Uremic plasma failed to induce inflammasome activation in b.End3 cells, while LPS (positive control) provoked a strong response. Studies analyzing the expression of tight junctions are ongoing and will be presented.

Conclusion: Our results demonstrate that uremic toxins impair the BBB independent of inflammasome activation.

\title{
DGKL-P060
}

\section{Evaluation of RealStar@ Influenza Screen\&Type RT-PCR Kit 4.0 for qualitative detection and differentiation of Influenza A/H1N1 and Influenza B specific RNA in respiratory samples}

\author{
Dietmar Wolff'; Karin Rottengatter'; Andreas Gerritzen ${ }^{3}$ \\ ${ }^{1}$ MVZ Medizinisches Labor Bremen GmbH, Molekularbiologie, Bremen, Germany; ${ }^{2}$ altona DIAGNOSTICS, scientific support, Hamburg,Germany; \\ ${ }^{3} \mathrm{MVZ}$ Medizinisches Labor Bremen GmbH, Geschäftsführung, Bremen
}

Objectives: The goal of the study is the evaluation of the performance of RealStar® Influenza Screen\&Type RT-PCR Kit 4.0 (altona Diagnostics, Germany) for the reliable detection of Influenza A and B specific RNA in respiratory samples after automated extraction with NucliSENS ${ }^{\circledR}$ easyMAG ${ }^{\circledR}$ system (bioMérieux) and amplification on LightCycler ${ }^{\circledR} 480$ System II (LC480, Roche). The evaluation is based on the comparison of performance data of RealStar ${ }^{\circledR}$ Influenza Screen\&Type RT-PCR Kit 4.0 and RIDA® GENE Flu (r-biopharm).

Methods: All assays were performed following the respective suppliers' recommendations. 10 QCMD 2017 Influenza virus A and B RNA EQA Program panel samples (Influenza A Challenge 1 and Influenza B Challenge 2) were used to show the general comparability in sensitivity and specificity of both assays.

In addition, 50 human clinical nasopharyngeal swabs were processed by the NucliSENS $₫$ easyMAG ${ }^{\circledR}$ system. Frozen stored retrospective positive and negative tested Influenza A and B (winter season 2017) routine samples were included into this study. All sample eluates were analyzed in parallel with RealStar ${ }^{\circledR}$ Influenza Screen\&Type RT-PCR Kit 4.0 and RIDA ${ }^{\circledR}$ GENE Flu real-time PCR on an LC480 instrument.

Results: 10 out of 10 QCMD samples showed concordant results by RealStar® Influenza Screen\&Type RT-PCR Kit 4.0 and RIDA®GENE Flu, respectively.

Qualitative testing of clinical respiratory samples with RealStar ${ }^{\circledR}$ Influenza Screen\&Type RT-PCR Kit 4.0 and RIDA® GENE Flu were generally concordant (90\%). 30 out of 50 samples were detected positive by both assays, 15 out of 50 samples were detected negative by both assays. 5 samples were detected positive by RealStar ${ }^{\circledR}$ Influenza Screen\&Type RT-PCR Kit 4.0 only, 2 cases Influenza A and 3 cases of Influenza B were missed by the RIDA®GENE Flu real-time PCR Kit.

Sequencing was used for further analysis of the discordant samples and to confirm the results.

Conclusions: Both assays show high concordance in sensitivity and specificity for Influenza A and B RNA detection in respiratory samples. Discordant results hint at the necessity of regular adaption of PCR systems to ensure highest sensitivity and specificity in routine diagnostics.

\section{DGKL-P061}

\section{Antiphospholipidantibodies differ in their dependency on the complementsystem}

Svenja Ritter ${ }^{1}$; Nadine Müller-Calleja ${ }^{1}$; Anne Hollerbach ${ }^{1}$; Karl Lackner' ${ }^{1}$, Wolfram Ruf ${ }^{2}$

${ }^{1}$ Unimedizin Mainz, Zentrallabor, Mainz, Germany; ${ }^{2}$ Unimedizin Mainz, Centrum für Thrombose und Hämostase, Mainz, Germany

Background: The Antiphospholipid-Syndrome (APS) is an autoimmune disease characterized by thrombosis or recurrent fetal loss associated with persistently elevated titers of antiphospholipid antibodies (aPL). Pathogenic effects of aPL have been linked to complement activation. Aims: We tested the hypothesis that complement activation by aPL is needed for their pathogenic role in the APS and if the pathway is generally applicable to all aPL.

Methods: We studied human monocytic cell activation by the monoclonal cofactor independent anti-cardiolipin aPL HL5B and the antiß2-glycoprotein I (B2-GPI)-aPL JGG9 by analysis of single stage clotting assay and phosphatidylserine exposure. We also evaluated the thrombus formation in B6, C3-/-, C5-/- and LRP8-/- mice after Injection of cofactor-independent HL5B.

Results: The anti-cardiolipin aPL was dependent on complement factor C3 to induce TF activation but was able to induce phosphatidylserine exposure in the absence of complement factor C5. The anti-ß2-GPI-aPL was not able to initiate either of those pathways.

Conclusions: Our results demonstrate that the collaboration of aPL with the complement system is quite different. And that the activation of monocytes by cofactor independent anti-cardiolipin aPL depends on complement activation especially C3, but phosphatidylserine exposure is independent on $\mathrm{C} 5$. 


\section{DGKL-P062}

\section{Reliable Quantification of Hepatitis D virus RNA independent of the genotype}

Eileen Brandenburger ${ }^{1}$; Birgit Bremer'; ${ }^{2}$ Michael Dietzsch'; André Reinhardt ${ }^{4}$; Ingolf Lachmann ${ }^{3}$; Heiner Wedemeyer ${ }^{5}$

${ }^{1}$ Analytik Jena, Produktmanagement, Jena, Germany; ${ }^{2}$ Medizinische Hochschule Hannover, Gastroenterologie, Hannover,Germany; ${ }^{3}$ AJ Roboscreen, F\&E, Leipzig, Germany; ${ }^{4}$ Analytik Jena, Vertrieb, Jena, Germany; ${ }^{5}$ Universitätsklinik Essen, Gastroenterologie und Hepatologie, Essen, Germany

The hepatitis D virus (HDV) is a small, enveloped RNA virus. At least eight genotypes have been identified. HDV, causing one of the most severe hepatic disease, infects individuals who suffer from hepatitis B infection. Worldwide the number of co-infected people exceeds 15 million. The only available treatment leading to suppression of HDV RNA is due to Interferon-alpha. Alternative treatments are currently under investigation. Nevertheless, confirmation of HDV infection, as well as standardized monitoring of viral load independent of the genotype, is urgently needed in order to personalize patient management. Corresponding routine tests are developed based on real-time PCR. For evaluation of the RoboGene ${ }^{\circledR}$ HDV RNA Quantification Kit 2.0 comparative analysis to an earlier published in-house method was performed. In addition the genetic variability of HDV detected by the kit was ascertained.

Methods: Samples of 15 HDV IgG-positive and 5 IgG-negative patients were analysed using the RoboGene® HDV RNA Quantification Kit 2.0 and an in-house method. Patient samples were tested before, as well as 12 and 48 weeks after treatment. Additionally, HDV positive samples of all different genotypes were examined. A serial 1:10 dilution of every genotype sample was prepared and compared to the routine assay of the French National Reference Laboratory.

Results: Before start of therapy, results obtained by the kit were consistent with those obtained by the in-house method. After 12 weeks treatment, $20 \%$ of patient samples were found to be negative with the new kit, in contrast to $33 \%$ when using the in-house method. After 48 weeks of treatment, $33 \%$ and $40 \%$ of samples tested negative with the kit and the in-house method, respectively. The Kit was able to detect all tested genotypes. According to the genotype panel the kit showed a very good repeatability, linearity and sensitivity. The RoboGene ${ }^{\circledR}$ kit was able to systematically detect and quantify very low loads of HDV RNA ( $>10 \mathrm{IU} / \mathrm{mL})$. The kit was also able to detect all strains of the panel, showing a good specificity for the different genotypes.

Conclusion: The results illustrate the usability of the RoboGene ${ }^{\circledR}$ HDV RNA Quantification Kit 2.0 for confirmation of HDV infections and determination of therapy efficiency. The kit is a reliable tool in investigating and quantifying patient samples offering excellent sensitivity, independent of the occurring genotype.

\section{DGKL-P063}

\section{Initial Reverse Transcription is essential for reliable Helicobacter pylori 235 rDNA Real-time PCR diagnostics from fecal samples}

Dietmar Wolff'; Andreas Gerritzen²

${ }^{1}$ Medizinisches Labor Bremen, Molekularbiologie, Bremen, Germany; ${ }^{2}$ MVZ Medizinisches Labor Bremen GmbH, Geschäftsführung, Bremen,

Objektives: A sensitive non-invasive molecular test for identifying Helicobacter pylori (H. pylori) in stool samples is important in the context of a "test-and-treat strategy". The goal of this study was to elucidate the benefit of an initial reverse transcription (RT) step for the sensitivity of H. pylori $23 \mathrm{~S}$ rDNA Real-time PCR from stool samples.

Methods: We compared a LightMix ${ }^{\circledR}$ Modular Helicobacter 23 S rDNA PCR without (LightMix ${ }^{\circledR}$ PCR) and with an initial reverse transcription step (LightMix ${ }^{\circledR}$ RT-PCR) assay for rapid detection of Helicobacter pylori from fecal samples. For maximum sensitivity all Lightmix ${ }^{\circledR}$ RT-PCR was performed in triplicate for each sample. Additionally the LightMix ${ }^{\circledR}$ RT-PCR was compared to H.pylori AG ELISA.

Results: Direct detection of H. pylori from feces by Lightmix ${ }^{\circledR}$ PCR was less sensitive than LightMix® RT-PCR. Only 2 out of 44 stool samples (4.5\%) from patients with diarrhea were positive for H. pylori in the LightMix ${ }^{\circledR}$ PCR assay. In contrast, a reverse transcription step prior Lightmix® PCR increased the assay sensitivity markedly (19 out of 44 positives, 43.2\%). When re-testing 65 samples initially analyzed with H.pylori AG ELISA with LightMix ${ }^{\circledR}$ RT-PCR, the detection rate for H. pylori was similarly increased from 23.1\% (15/65) with H.pylori AG ELISA to 43.1\% (28/65) with H. pylori LightMix ${ }^{\circledR}$ RT-PCR. $21.5 \%$ of the $28 \mathrm{H}$. pylori positive samples were positive in $1 / 3,32 \%$ in $2 / 3$ and $46.5 \%$ in $3 / 3$ triple RT-PCR approaches, respectively. Re-testing the 28 LightMix ${ }^{\circledR}$ RT-PCR positive samples by LightMix ${ }^{\circledR}$ PCR, only $17.9 \%$ (5/28) RT-PCR positive samples were positive in PCR.

Conclusions: LightMix ${ }^{\circledR}$ RT-PCR is much more sensitive than Lightmix ${ }^{\circledR}$ PCR. In comparison to H.pylori AG ELISA the LightMix ${ }^{\circledR}$ RT-PCR shows a markedly higher sensitivity. An initial reverse transcription step is crucial for reliable Helicobacter pylori 23S rDNA Real-time PCR diagnostics. Triple RT-PCR approaches can additionally improve the detection of H. pylori out of fecal samples. 


\title{
DGKL-P064
}

\section{CXCL13 and the Differential Diagnosis of Neuroborreliosis: Very High Level of CXCL13 in the Cerebrospinal Fluid in a Patient with Presumed Bing-Neel Syndrome (CNS Manifestation of Waldenstrom's Disease)}

\author{
Joachim Kappler'; Christoph Splittstößer²; Daniel Himmelreich'; Monika Bös²; Renate Reichwein-Kappler'; Kley Christoph ${ }^{2}$ \\ ${ }^{1}$ MVZ SYNLAB Leverkusen, Laboratoriumsmedizin, Leverkusen, Germany; ${ }^{2}$ GFO Kliniken Troisdorf, Neurologie, Troisdorf, Germany
}

CXCL13 (formerly known as B lymphocyte chemoattractant, BLC) is a chemokine that regulates B-cell homing and the formation of secondary lymphoid tissues via its receptor CXCR5. An elevated concentration of CXCL13 in the cerebrospinal fluid (CSF) is an established criterion of B cell activation in neuroborreliosis and other spirochetal CNS diseases (neurolues). Inflammatory CSF Syndrome with lympho-monocytic pleocytosis, impairment of the blood-brain barrier and predominant intrathecal IgM synthesis is typically found in acute neuroborreliosis. An elevated CXCL13-level above $300 \mathrm{pg} / \mathrm{ml}$ (normal range $<20 \mathrm{pg} / \mathrm{ml}$ ) in a patient presenting with typical symptoms is suggestive of acute neuroborreliosis even in the absence of intrathecal production of borrelia-specific antibodies at an early disease stage. However, elevated CSF CXCL13 was reported in CNS lymphomas and some other conditions.

Here we report on a male patient aged 84 years intitially presenting with an acute delirant episode, mild cognitive impairment and mild axonal polyneuropathy as well as a gait disorder and an anamnesis of Waldenstrom's disease that was treated with 6 cycles Rituximab/ Ribomustin. Relevant laboratory findings on first admission were 1. a monoclonal gammopathy of the type IgM kappa. 2. a severely disturbed blood-brain barrier (QAlb $=51.1)$ without indication of intrathecal antibody production. 3. a mild lymphocytic pleocytosis $(16$ cells/ $\mu \mathrm{l})$ and 4 . a weakly positive screening test for borrelia-specific IgG in Serum. Direct and indirect CSF testing for HSV-1, HSV-2 and VZV was negative. There was neither elevation of tau and phospho tau levels nor reduced beta-amyloid peptide 1-42 in CSF.

One month later QAlb was 42.5 and borrelia-specific IgG-AIs were weakly positive (lysate 0.66, p100 2.35, p18 0.86, p39 2.50; Diamex particle assay). The CXCL13 level was 55,200.0 pg/ml (Euroimmun). Thus, the patient was treated with Ceftriaxone over 2 weeks. Examination of the CSF during treatment revealed QAlb $=50.8$ and a drop of CXCL13 $=14,595.0 \mathrm{pg} / \mathrm{ml}$, but re-examination after treatment showed QAlb $=52.4$ and a raise of CXCL13 to $19,907.0 \mathrm{pg} / \mathrm{ml}$. On clinical examination the gait disorder had worsened and impaired cognitive parameters were unchanged. Thus, there was no clinical response to antibiotic treatment and the diagnosis of neuroborreliosis was abandoned.

In summary, the present case suggests that very high levels of CXCL13 in CSF may be caused by CNS manifestation of Waldenstrom's disease (Bing-Neel syndrome).

\section{Posterwalk Inflammation, Infektion und Autoimmunerkrankungen II}

\section{DGKL-P065}

\author{
The STAT6 target ephrin-A1 influences M2a polarization and migration of human macrophages
}

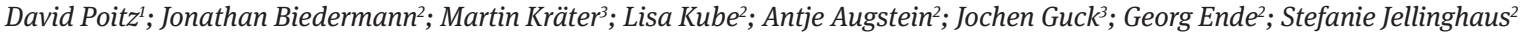 \\ ${ }^{1}$ TU Dresden, Institut für klinische Chemie und Laboratorium, Dresden, Germany; ${ }^{2}$ TU Dresden, Klinik für Innere Medizin und Kardiologie, \\ Dresden, Germany; ${ }^{3}$ TU Dresden, Biotechnologisches Zentrum, Dresden, Germany
}

\begin{abstract}
Introduction: Macrophages are central players in the initiation and progression of atherosclerosis. Beside classically activated (M1) also alternatively activated (M2) macrophages were detected within atherosclerotic plaques. In contrast to the pro-inflammatory phenotype of M1 macrophages, M2 macrophages display a more anti-inflammatory phenotype. In recent years the Eph/ephrin-system, the largest system of receptor tyrosine kinases, came into focus for the process of atherosclerosis. We and other could show that it is involved in the interaction between monocytes and endothelial cells as well as between the endothelial cells itself.

Aim: The aim of the present study was to investigate the regulation and function of the Eph/ephrin-system in human macrophages. Methods: Human monocyte-derived macrophages (M0) were polarized towards the M1 and M2a phenotype using IFN /LPS or IL-4.

Results: In a first attempt these three phenotypes were screened for the expression of all Eph-receptors and ephrin-ligands. Showing a defined regulation of the Eph/ephrin-system in different macrophage subtypes, the most pronounced effect was seen in case of ephrin-A1. The expression of ephrin-A1 (mRNA, protein, surface protein) was highly induced in M2a macrophages. This induction is mediated by the transcription factor STAT6 as proven by ChIP and siRNA-silencing. Silencing of ephrin-A1 revealed a strongly reduced expression of several typical M2 genes like M2 polarization marker MRC1 or STAT6, the central transcription factor in M2 polarization. This suggests that ephrinA1 modulates macrophage phenotype itself. Moreover, we could show that polarization as well as ephrin-A1 influence cell mechanics of human macrophages. Performing transwell migration assays, we detected higher migration rates of M2a macrophages compared to M0 macrophages. Silencing ephrin-A1 decreased migration rate in both M0 and M2a macrophages. Applying real-time deformability cytometry, we could show that this difference in migration might be due to a modulation of the cell deformability. The deformability of macrophages was increased in M2a polarized macrophages and decreased after silencing of ephrin-A1. Proofing ephrin-A1 expression within atherosclerotic
\end{abstract}


plaques, aortae of 1yr old ApoE-/- mice were explanted. Analysis revealed a higher mRNA expression of ephrin-A1 in plaque regions, which nicely correlated with the expression of macrophage marker CD68.

Conclusion: Taken together, ephrin-A1 is expressed within the atherosclerotic plaque and influences the M2 polarization as well as migration capacity of human macrophages. The importance of migration as a precondition for effective phagocytosis and the possible protective function of M2 macrophages in atherosclerotic plaques, make ephrin-A1 to an intriguing target to analyze for its atherogenic potential.

\section{DGKL-P066}

\section{The secreted protein Del-1 promotes resolution of inflammation}

Ioannis Kourtzelis ${ }^{1}$; Xiaofei Li²; Tetsuhiro Kajikawa ${ }^{2}$; Ioannis Mitroulis ${ }^{1}$; Daniel Grosser ${ }^{3}$; Baomei Wang ${ }^{2}$; Michal Grzybek ${ }^{3}$; Janusz von Renesse ${ }^{1}$; Maria Troullinaki ${ }^{1}$; Klara Ruppova ${ }^{1}$; Sylvia Grossklaus ${ }^{1}$; Leo A.B. Joosten ${ }^{4}$; Niki M. Moutsopoulos ${ }^{5}$; Ben Wielockx ${ }^{1}$; Antonio Castrillo ${ }^{6}$; Jonathan M. Korostoff'; Uenal Coskun ${ }^{3}$; George Hajishengallis²; Triantafyllos Chavakis ${ }^{1}$

${ }^{1}$ TU Dresden Institut für klinische Chemie und Laboratorium Medizin, -, Dresden, Germany; ${ }^{2}$ University of Pennsylvania, Penn Dental Medicine, Department of Microbiology, -, Philadelphia, United States; ${ }^{3}$ Paul Langerhans Institute Dresden of the Helmholtz Zentrum München at the University Hospital \& Faculty of Medicine Carl Gustav Carus of TU Dresden, -, Dresden, Germany; ${ }^{77 D e p a r t m e n t ~ o f ~ I n t e r n a l ~ M e d i c i n e ~ a n d ~ R a d b o u d ~}$ Center for Infectious Diseases, Radboud University Medical Center, -, Nijmegen, Netherlands; ${ }^{5}$ Oral Immunity and Inflammation Unit, NIDCR, NIH, -, Bethesda, United States; 'Instituto de Investigaciones Biomedicas "Alberto Sols" Consejo Superior de Investigaciones Científicas (CSIC) de Madrid, Spain and Unidad de Biomedicina IIBM-ULPGC (Unidad Asociada al CSIC), Instituto Universitario de Investigaciones Biomedicas y Sanitari, -, Las Palmas, Spain; 'University of Pennsylvania, Penn Dental Medicine, Department of Periodontics, -, Philadelphia, United States

Acute inflammation is initiated by neutrophil and monocyte recruitment to the inflamed site, followed by release of pro-inflammatory mediators. After their acute proinflammatory actions, recruited neutrophils undergo apoptosis and are phagocytosed by macrophages, which is mediated by recognition of the "eat-me" signal phosphatidylserine (PS) on apoptotic neutrophils and specific macrophage receptors. This process is called "efferocytosis". Macrophage engulfment of the apoptotic material leads to the induction of a downstream signaling in which efferocytic macrophages acquire a resolving phenotype by releasing TGF $\beta 1$ and by further upregulation of phagocytic receptors and bridging molecules. Resolving macrophages are central to resolution of inflammation, which is essential for tissue homeostasis. We have previously described Developmental endothelial locus-1 (Del-1) as an endogenous negative regulator of leukocyte adhesion by blocking the interaction between the integrin LFA-1 and ICAM-1.

To investigate the role of Del-1 in the modulation of inflammation resolution, we employed two different animal disease models, used genetic and pharmacological approaches and also analyzed relevant human samples.

In this report, we show that Del-1 also operates as an endogenous pro-resolving mediator. In human and murine oral inflammatory disease, periodontitis, resolution of inflammation correlated with Del-1 upregulation, whereas resolution of experimental periodontitis failed in Del-1 deficiency. This concept was generalized and mechanistically substantiated in acute monosodium urate crystal-induced inflammation, where the pro-resolution function of Del-1 was attributed to effective apoptotic neutrophil clearance (efferocytosis). The C-terminal discoidin-I-like domains of Del-1 were strictly required for efferocytosis owing to their high-affinity binding to PS. Consistent to its ability to resolve inflammation, Del-1-mediated efferocytosis induced macrophage reprograming to a resolving phenotype in a manner dependent on nuclear receptor liver X receptor. Importantly, by generating different transgenic mice with cell-specific overexpression of Del-1, we ascribed the anti-leukocyte recruitment action to endothelial cell-derived Del-1 and the efferocytic/pro-resolving action to macrophage-derived Del-1.

Therefore, the homeostatic function of Del-1 is not restricted to regulating initiation of inflammation but also encompasses its resolution and this versatility is facilitated by the compartmentalized expression of Del-1 that enables it to perform distinct functions in an appropriate context that can be harnessed therapeutically.

\section{DGKL-P067}

\section{Activity-based quantification of neutrophil granule-associated serine proteases}

Eva Riedlinger; Fabian Ullich; Erika Siepmann; Stefan Simon; Christian P. Sommerhoff

Institute of Laboratory Medicine, University Hospital, LMU Munich, AG Clinical Biochemistry/Proteolysis, Munich, Germany

Objective: Granule-associated serine proteases of immune defense (GASPIDS) are a family of multifunctional proteases that are indispensable for the regular function of the immune system. When excessively released and active, however, GASPIDS contribute to the development of various inflammatory and degenerative disorders. Neutrophils contain large quantities of neutrophil elastase, proteinase 3, cathepsin G and NSP4 (neutrophil serine protease 4) that are stored in azurophilic granules after being activated by the cysteine proteinase cathepsin C. Current immunoassays do not discriminate between the mature, active protease and inactive forms, e.g. the zymogen and protease/inhibitor complexes. Activity-based assays therefore seem to be more appropriate to quantify the active and pathophysiologic relevant form of neutrophil GASPIDS. 
Methods: Polymorphonuclear neutrophils were isolated from whole blood samples from healthy volunteers ( $\mathrm{n}=28$, age $25 \pm 4.4$ years) by immunomagnetic depletion. Purity and residual erythrocytes were assessed by cytospin and Harboe spectrophotometric method, respectively. The enzymatic activity of neutrophil elastase, proteinase 3, cathepsin $\mathrm{G}$ and cathepsin $\mathrm{C}$ was quantified in neutrophil lysates using kinetic assays with fluorogenic and chromogenic peptide substrates.

Results and discussion: Purity of isolated neutrophils was $98 \pm 0.45 \%$ of recovered leucocytes. With intra-assay CVs of $\leq 10 \%$, fluorogenic kinetic assays were better suited to quantify the activity of GASPIDS than chromogenic assays. Protease activity was stable throughout a freeze/thaw cycle and short-term storage at $80^{\circ} \mathrm{C}$ with recoveries $\geq 90 \%$. The assays were linear and selective as verified e.g. using isolated or recombinant proteases and protease inhibitors.

These novel activity assays thus appear adequate for quantifying GASPIDS and their activator cathepsin C in crude neutrophil lysates. Only $\sim 20,000$ neutrophils usually present in $<20 \mu \mathrm{l}$ of whole blood are sufficient for all measurements. These assays therefore may advance investigations on the role of GASPID in immune defense and inflammatory disorders.

Supported by DFG Priority Program 1394 “Mast Cells - Promoters of Health and Modulators of Disease”.

\section{DGKL-P068}

\section{Entstehung von Antiphospholipid Antikörpern}

Nadine Müller-Calleja; Carolin Orning; Karl Lackner

Universitätsmedizin Mainz, Institut für klinische Chemie \& Laboratoriumsmedizin, Mainz, Germany

Background and Aim of Study: The antiphospholipid syndrome (APS) is an autoimmune disease characterized by thromboembolic events and/or abortions in the presence of antiphospholipid antibodies (aPL). It is well accepted that aPL are causally involved in the pathogenesis of APS. However, the origin of aPL remains obscure. It is also unclear why transient aPL titers occur in in some patients, while other patients develop persistent aPL titers and clinical manifestations of APS. In the present study we focus on the question how pathogenic aPL arise in a peculiar mouse model of immunization-induced aPL. In this model immunization with an aPL induces production of aPL in the immunized mouse.

Methods: To induce aPL, wild type and different knockout mouse strains were immunized in the footpads with human monoclonal aPL together with Freund's adjuvans. APL titers were measured by ELISA over a period of 12 weeks from the sera of these animals.

Results: Immunization of WT mice with a human anti-cardiolipin aPL resulted in elevated mouse anti-cardiolipin (aCL) IgG titers. This increased aCL IgG production was already detectable 2 weeks after immunization, supporting the idea that aPL belong to the group of natural antibodies i.e. that aPL specific preformed B-cell clones exist. Interestingly, immunization with aCL also induced production of anti-b2GPI antibodies but at a much later point in time indicating an epitope maturation process. Immunization of knockout mice revealed that aCL induction was independent of MHCII but depended on NADPH-oxidase and TLR7 both involved in signaling of the aPL used for immunization. Conclusion: In our mouse model aPL were produced by preformed B-cell clones activated via TLR7 and NOX2 in a T-cell independent manner. Further work to identify the aPL-producing B-cells is under way.

\section{DGKL-P069}

\section{DHEA-Del-1 axis: an immune-endocrine homeostatic pathway}

Athanasios Ziogas ${ }^{1}$; Vasileia-Ismini Alexaki ${ }^{1}$; Markus Sperandio ${ }^{2}$; George Hajishengallis ${ }^{3}$; Triantafyllos Chavakis ${ }^{1}$

${ }^{1}$ TU Dresden, Institut für klinische Chemie und Laboratorium, Dresden, Germany; ${ }^{2}$ Ludwig-Maximilians-Universität München, Walter Brendel Centre of Experimental Medicine, Munich, Germany; ${ }^{3}$ University of Pennsylvania School of Dental Medicine, Department of Microbiology, Philadelphia, United States

Objectives: Del-1 is an endothelial secreted glycoprotein with anti-inflammatory properties and little is known about the expressional regulation of this molecule. Dehydroepiandrosterone (DHEA) is the most abundant circulating steroid hormone in humans. The levels of DHEA have been noted reduced in subjects with autoimmune diseases, sepsis and trauma, but its role in inflammation remains elusive. In the present study we studied the potential role of DHEA in leukocyte recruitment and tried to understand the underlying mechanisms.

Methods: To examine whether DHEA has a potential regulatory role in inflammatory cell recruitment and adhesion in vivo, we engaged endotoxin (LPS)-induced lung inflammation and performed intravital microscopy by using the cremaster model in mice. The effect of DHEA on the expression of endothelial derived adhesion molecules as well as on $\beta 2$ integrin-dependent leukocyte adhesion was also evaluated in vitro.

Results: In the model of LPS-induced lung inflammation, DHEA treatment inhibited neutrophil extravasation. Furthermore, intravital microscopy revealed that DHEA impaired LPS-induced leukocyte adhesion efficiency and thereby increased rolling velocity. In vitro studies revealed no effect of DHEA on $\beta 2$ integrin activation or endothelial cell adhesion molecule expression. Instead, we found that DHEA counteracted the TNF-dependent downregulation of endothelial Del-1 expression. In particular, TNF-mediated inhibition of endothelial Del-1 expression 
involved the inhibition of C/EBP $\beta$ transcription factor recruitment to EDIL3 promoter. This deactivation of Del-1 expression was reversed by DHEA, in a manner dependent on the endothelial TrkA receptor and Akt-signaling as well as by NGF, the known ligand of TrkA receptor. The anti-inflammatory effect of DHEA in the model of LPS-induced lung inflammation was completely abrogated in Del-1 KO mice as well as in mice with endothelial specific deletion of TrkA, thus, demonstrating that DHEA exerts its anti-inflammatory actions mainly through TrkA signaling and Del-1 regulation.

Conclusion: Our results identify a novel anti-inflammatory effect of DHEA that blocks leukocyte adhesion and leukocyte recruitment, by restoring the levels of the local homeostatic factor Del-1.

\section{DGKL-P070}

\section{A new LC-MS/MS -method for simultaneous quantification of carboxy-terminal alpha1-antitrypsin peptides}

Claudia Schnabel; Nancy Blaurock; Dominic Walter; Michael Kiehntopf

Universitätsklinikum Jena, Institut für Klinische Chemie und Laboratoriumsdiagnostik, Jena, Germany

Objective: Actually procalcitonin is the most widely used biomarker to differentiate between infectious and non-infectious systemic inflammation. However, especially in critically ill patients the discriminatory potential of procalcitonin is only moderate due to limited specifity. However, in a recent study using mass spectrometry, the discriminatory potential of CAAP48 (carboxy-terminal alpha1-antitrypsin peptide, $4789 \mathrm{Da}$ ) and its polymorphism CAAP47 (4775 Da), due to the frequent SNP (rs1303, MAF 0.28) leading to an E > D substitution, was shown to be superior to procalcitonin. According to the MEROPS database further potential carboxy-terminal cleavage sites of AAT might be of interest in this setting. Thus, we established a mass spectrometry method for the simultaneous quantification of the carboxy-terminal alpha1-antitrypsin peptides with a length of 45aa, 44aa, 43aa, 42aa (CAAP47/48), 40aa, 39aa, 37aa, 36aa and 22aa and their SNP-variants.

Materials and methods: The method was developed using an API4000 ${ }^{\mathrm{TM}}$ mass spectrometer (AB SCIEX, Framingham, MA, USA) equipped with an electrospray ionization source connected to a liquid chromatography system LC20 AVP (Shimadzu, Japan) consisting of a thermostatic autosampler, a binary solvent delivery manager and a thermostated column compartment. Separation of the peptides was achieved by using a Kromasil-Eternity - 2.5 - C18 2.1 x 100 mm UHPLC column (Dichrom GmbH, Marl, Germany) precoupled with a KruckKatcher Ultra HPLC In-Line Filter $0.5 \mu \mathrm{m}$ (Phenomenex, USA). Analytical standard material of all peptides (purity of $>95 \%$ ) and of 13C6/15N - leucine- labeled internal standards (CAAP47/48, C37 and C22) were purchased by EMC microcollections (Tübingen, Germany) and Smart Bioscience (Saint Egrève, France), respectively. Peptides were dissolved in a 1:1 mixture of Acetonitril (+ formic acid 0.1\%) and H2O (+ formic acid 0.1\%). For each peptide two transitions for mother and daughter ions were evaluated: one for quantification (quantifier) and one for confirmation (qualifier), furthermore for each transition compound specific instrument parameters were optimized to obtain the most robust and intensive signal.

Results: All peptides and their SNP-variants can be detected in PBS-buffered albumin ( $50 \mathrm{~g} / \mathrm{l})$ as well as in human plasma at a concentration of $10 \mathrm{mg} / \mathrm{ml}$ with appropriate intensities. Further validation and characterization of the method according to EMA is under way.

Summary: The method developed gives the opportunity for rapid analysis of AAT-peptides and thus allows further clinical validation of c-terminal AAT-peptide patterns, as biomarkers for discrimination of infectious and non-infectious systemic inflammation.

\section{DGKL-P071}

\section{(Phospho-)Proteome analysis of TNF long term-incubated primary human monocytes}

Rolf Bikker'; Bastian Welz'; Martin Christmann ${ }^{1}$; Mareike Weber ${ }^{1}$; Leonie Hoffmeister ${ }^{1}$; Konstantin Neumann ${ }^{1}$; Katharina Preu $\beta^{1}$; Johannes Junemann'; Andreas Pich'; René Huber'; Korbinian Brand ${ }^{1}$

${ }^{1}$ Medizinische Hochschule Hannover, Institut für Klinische Chemie, Hannover, Germany;

${ }^{2}$ Medizinische Hochschule Hannover, Institut für Toxikologie, Hannover

Objective: The precise regulation of the inflammatory process is essential for its termination since dysregulations may result in acute/chronic inflammatory diseases or immune paralysis. To better understand the inflammation-associated mechanisms modulating and terminating TNF-induced signal transduction after stimulation, we analyzed both proteome and phospho-proteome in TNF long term-incubated primary human monocytes.

Methods: For the isolation of primary human monocytes from blood samples of healthy donors, a negative cell isolation protocol was applied. Monocytes were incubated $\pm 400 \mathrm{U} / \mathrm{ml} \mathrm{TNF}$ for $48 \mathrm{~h}$, protein extracts were prepared ( 2 donors each, $\mathrm{n}=4$ ), and phosphorylated proteins were enriched via IMAC and TiO2 columns. Subsequently, a systemic analysis of the (phospho )proteome was performed using liquid chromatography/mass spectrometry. (Phospho )proteins were identified using MaxQuant. For data analysis and visualization, the Perseus software was used.

Results: Our (Phospho-)proteome analysis revealed 148 significantly regulated proteins (103 proteins increased, 45 proteins decreased) and 569 significantly regulated phophopeptides (377 peptides with increased, 192 peptides with decreased phosphorylation) in long term 
TNF-treated samples (cut off: 2- and 0.5-fold induction, respectively). Among the significantly regulated (phospho-)proteins several promising candidates were identified. Mechanistically, a variety of these proteins is involved in the negative regulation of the NF- $\mathrm{kB}$ system (including NFKB1, NFKB2, RelB, and CEBPB) and possesses distinct binding motifs for kinases known for their contribution to the regulation of proinflammatory signaling such as GSK3.

Conclusion: Our experiments indicate that a (phospho-)proteome analysis of primary monocytes can be successfully applied yielding the identification of a defined set of proteins characterized by reproducible changes in expression and phosphorylation patterns. We conclude that our analyses provide new candidates as well as indication for a complex network of specific but synergistically acting and cooperating mechanisms enabling the affected cells to resist sustained TNF exposure and potentially also influencing the resolution of inflammation.

\title{
DGKL-P072
}

\section{Fecal Calprotectin by turbidimetric method - impact on the Laboratory workflow}

\author{
Luca Germagnoli \\ Synlab Italy, Core Lab, Synlab Italia Castenedolo, Italy
}

Background: Calprotectin (Cal) is a cytoplasmic protein mainly derived from neutrophilic granulocytes. It is considered as a high sensitive inflammation marker. Fecal $\mathrm{Cal}$ (fCal) is well established as an aid in diagnosis of chronic inflammatory bowel disease (IBD) to differentiate between inflammatory and non-inflammatory (e.g. IBS) diseases. As well as for monitoring and therapy follow up of IBD patients.

Question: What is the impact on the workflow using the BÜHLMANN turbidimetric method.

Material and Methods: The BÜHLMANN fCAL ${ }^{\circledR}$ turbo can be applied on most clinical chemistry analyzers. At Synlab Italy this method runs on Beckman AU5800 and replaced the manual ELISA method (Calprest, Eurospital). The Synlab laboratory in Castenedolo is the largest laboratory of the Italian Synlab network; in total it processes about 15 Mio tests per year. To perform a workflow analysis with the two fCal methods we chose the time periods May-Dec2016 (Caprest, $\mathrm{n}=12945$ ) and May-Dec2017 (Buhlmann fCAL, $\mathrm{n}=16538$ ) and we assumed that the number of customers remained stable. A comparison between the TAT from laboratory check-in to results and from check-in to reports signature was done. Additionally, a method comparison was done.

Results: The fCAL turbo method allowed a daily fCal routine activity in our lab. With the manual ELISA method the median time to result was 74.4 hours and 75\% (3rd Quartile) of all requests were completed after 115.1 hours. With the turbidimetric test a significant reduction was achieved. With a median of 29.4 hours (3rd quartile of $52.3 \mathrm{~h}$ ). Consequently, the time to signature/report was significantly reduced from 86.3 hours (median ELISA) to 36.4 hours (median fCAL turbo). The indicated TAT includes the transportation time to Castenedolo where the fCal method is centralized for the whole country. Transportation varies from a few hours to about 12hours for the patients living in south Italy. Regarding to fCal results the relative amount of negative results ( $<50 \mu \mathrm{g} / \mathrm{g}$ ) was $76.9 \%$ for the Calprest ELISA and changed to $56.3 \%$ with fCAL turbo; positive results where $13.9 \%$ for Calprest ELISA ( $>100 \mathrm{ug} / \mathrm{g}$ ) and $17.4 \%$ for fCAL turbo ( $>200 \mathrm{ug} / \mathrm{g}$ ). The Authors made a previous clinical evaluation of turbidimetric method, and $89.2 \%$ of the patients had the same classifications by both assays with 4 real discrepancies.

Discussion and conclusions: The fCAL turbo is a highly accurate and clinically proven method. The introduction of the turbidimetric method on a high throughput analyzer allowed a very fast closing of our requests and it did not cause any significant problems for the instruments or difficulties for the technicians involved. The introduction of a quantitative extraction device could further streamline the work flow in the laboratory and circumvent the laborious and time consuming extraction itself.

\section{DGKL-P073}

\section{Impact of the ubiquitin system on the orchestration of signal transduction during continuous TNF challenge}

\author{
Martin Christmann; Rolf Bikker; Korbinian Brand \\ Medizinische Hochschule Hannover, Institut für Klinische Chemie, Hannover, Germany
}

Tumor necrosis factor (TNF) is a master cytokine orchestrating inflammatory response in humans. It exhibits pro-inflammatory functions but is also required for a proper termination of the immune response. The coordination of TNF signaling requires various posttranslational modifications. Disturbed TNF signal perception is a hallmark of numerous human diseases, such as SIRS and Sepsis or chronic inflammatory maladies like rheumatoid arthritis or inflammatory bowel disease as well as malignant processes. Current data indicate that a prolonged challenge of monocytic cells with TNF is associated with a deranged signal transmission culminating in an aberrant termination of pro-inflammatory signals. The preliminary experiments performed in preparation of this grant proposal revealed a significant reduction of phosphorylation at serin-20 within ubiquitin after long-term TNF exposition. In addition, a permanent TNF challenge appeared to be associated with recognizable changes of the ubiquitin system. The scope of this project will be to explore the role of ubiquitin (Ub) and ubiquitinated proteins for the orchestration of the proper sequence of TNF-induced events during prolonged challenge with the cytokine. (1) Thus, we will explore the biologic function of the rarely studied phosphorylation site at serin-20 of ubiquitin in terms of TNF-mediated signaling. (2) Furthermore, 
we aim to investigate the change in ubiquitinated substrates along the course of a steady TNF signal. Therefore, we will enrich and identify the pool of ubiquitinated proteins at distinct time points. Finally a more comprehensive picture of the consequences of ubiquitin regulation during permanent TNF challenge will be obtained. Such a systematic analysis of ubiquitin function during TNF signal transduction may help to improve diagnosis and prognosis of inflammatory diseases as well as malignant processes and will allow the development of more subtle therapeutic strategies.

\title{
Therapeutisches Drug Monitoring und Pharmakogenetik I
}

\section{DGKL-P074 / PV11}

\author{
The effect of genetic variants in DPYD and miR-27a on uracil metabolism and liver DPD activity.
}

Seid Hamzic ${ }^{1}$; Dominic Schärer'; Stefano Fontana ${ }^{2}$; Ursula Amstutz'; Carlo Largiadèr ${ }^{1}$

${ }^{1}$ Inselspital, Universitätsspital Bern, Universitätsinstitut für klinische Chemie, Bern, Switzerland; ${ }^{2}$ Interregionale Blutspende SRK AG, Interregionale Blutspende SRK AG, Bern, Switzerland

\begin{abstract}
Aims: The enzyme dihydropyrimidine dehydrogenase (DPD) is the key metabolizing enzyme of the cancer drug 5-Fluorouracil (5-FU). During the last years, four DPD impairing variants (c.1905+1G > A, c.1679T > G, c.2846A > T and c.1129-5923C > A) in the DPD encoding gene (DPYD) have emerged as useful markers for the prediction of 5-FU related toxicities. However, other DPYD variants e.g. c.496A $>$ G and c.1601G $>A$, and a variant (n.40A $>\mathrm{G}$ ) in the DPD regulating microRNA miR-27a sus-pected to confer lower DPD activity require further assessment of their clinical relevance. Therefore, we assessed the impact of these polymorphisms together with the four clinically relevant DPYD vari-ants on endogenous uracil (U), dihydrouracil (UH2) plasma levels and DPD activity in human liver. Methods: Endogenous U and UH2 plasma levels were assessed in two cohorts of healthy volunteers $(\mathrm{N} 1=320$ and $\mathrm{N} 2=308)$ and in a small cohort of 5 -FU treated patients $(\mathrm{N}=24)$ by LC-MS/MS. DPD activi-ty was measured in human liver S9 fractions by a LC-MS/MS assay $(\mathrm{N}=25)$. All samples were geno-typed for the same DPYD and miR-27a variants. Results: As expected, all clinically relevant DPYD variants were associated with lower UH2/U ratios in healthy volunteers (Pcombined < 0.0001). Homozygous or compound heterozygous carriers of DPYD c.496G and c.1601A were also associated with de-creased UH2/U ratios (Pcombined_hom = 0.0008). Surprisingly, miR-27a n.40G was associated with higher UH2/U ratios $($ Pcombined $=0.0036$ ) in the subgroup of DPYD risk variant carriers, whereas carriers of this variant showed a trend to lower UH2/U ratios in 5-FU treated patients. Additionally, homozygous n.40G carriers showed a trend to $10 \%$ lower DPD activity in human liver samples as compared to n.40A car-riers. Conclusion: Our results suggest that homozygous or compound heterozygous carriers of c.496G and c.1601A have impaired DPD activity. For the miR-27a n.40G variant, conflicting results were obtained. Interestingly, a trend in the expected direction was observed in liver tissue, a major site of DPD activity, an under 5-FU treatment, where the enzyme reaches a saturated state due to the exposure to high drug concentrations.
\end{abstract}

\section{DGKL-P075}

\section{Apixaban and rivaroxaban plasma concentrations in super obese patients - a case series}

Martin Wiesen ${ }^{1}$; Carsten Müller'; Thomas Streichert²; Guido Michels ${ }^{3}$; Cornelia Fietz ${ }^{1}$

${ }^{1}$ Universitätsklinikum Köln, Zentrum für Pharmakologie - TDM, Köln, Germany; ${ }^{2}$ Universitätsklinikum Köln, Institut für Klinische Chemie, Köln,Germany; ${ }^{3}$ Universitätsklinikum Köln, Innere Medizin III, Kardiologie, Herzzentrum, Köln, Germany

Background: According to guideline recommendations of the International Society on Thrombosis and Haemostasis, direct oral anticoagulants (DOACs) should not be used in obese patients with a weight $>120 \mathrm{~kg}$ or a BMI $>40 \mathrm{~kg} / \mathrm{m} 2$ owing to uncertainties about their safety and efficacy this group of patients. If DOACs are used nevertheless, treatment should be accompanied by monitoring of DOAC-specific trough and peak plasma concentrations to assure that levels lie within expected on-therapy concentration ranges.

Aim: We assessed plasma concentrations of apixaban and rivaroxaban to address concerns about underdosing super obese patients treated with standard doses of DOACs.

Material and Methods: Four patients were included at the Department III of Internal Medicine, Cardiology, Heart Center, University Hospital of Cologne. Two patients were diagnosed with non-valvular atrial fibrillation and two patients received treatment for recurrent pulmonary embolism. Plasma concentrations of apixaban and rivaroxaban were measured by a validated liquid-chromatography coupled to tandem mass spectrometry method.

Results: The patients' body weight ranged between $140.0 \mathrm{~kg}$ and $229.5 \mathrm{~kg}$ and body mass indices ranged from $50.7-66.5 \mathrm{~kg} / \mathrm{m}^{2}$. Three patients received apixaban with doses of $5 \mathrm{mg}$ or $10 \mathrm{mg}$ twice daily and one patient was treated with rivaroxaban 20 mg once daily. Median (range) trough and peak apixaban concentrations were $80.5 \mathrm{ng} / \mathrm{ml}(53.2-328.2 \mathrm{ng} / \mathrm{ml})$ and $236.2 \mathrm{ng} / \mathrm{ml}(125.2-613.6 \mathrm{ng} / \mathrm{ml})$. Observed rivaroxaban trough and peak levels were 111.2 and $230.1 \mathrm{ng} / \mathrm{ml}$, respectively. No thromboembolic complications occurred during the clinical course. 
Conclusion: In this small cohort of patients with super obesity, apixaban and rivaroxaban plasma concentrations were found within expected on-therapy ranges. Based on these results, the patients continued to receive apixaban or rivaroxaban. More studies are warranted to assess the safety and efficacy of DOACs in patients with super obesity.

\title{
DGKL-P076
}

\section{Comparison between direct measurements of DOACs by online SPE-UPLC-MS/MS and by functional based coagulation assays}

Joachim Kuhn'; ${ }^{1}$ Doris Hendig² ${ }^{2}$ Isabel Faust² ${ }^{2}$ Cornelius Knabbe²; Ingvild Birschmann ${ }^{2}$

${ }^{1}$ Herz- und Diabeteszentrum Nordrhein-Westfalen, Institut für Laboratoriums- und Transfusionsmedizin, Bad Oeynhausen, Germany; ${ }^{2}$ Herzund Diabeteszentrum NRW, Universitätsklinik der Ruhr-Universität Bochum, Institut für Laboratoriums- und Transfusionsmedizin, 32545 Bad Oeynhausen, Germany, Bad Oeynhausen, Germany

Objective: Measurement of direct oral anticoagulants (DOACs) concentration in patient blood is essential in special clinical circumstances. Issue: How good is the correlation between direct and functional DOACs measurements?

Methods: Direct measurements of DOACs were performed using our selective and sensitive automated online SPE-UPLC-MS/MS assay. The results of the online SPE-UPLC-MS/MS sample measurements were compared with the results of commercially available coagulation assays for the DOACs using a total of 120 clinical samples ( 45 samples from patients which were treated with apixaban, 27 samples from patients which were treated with dabigatran, 8 samples from patients which were treated with edoxaban, and 40 samples from patients which were treated with rivaroxaban).

Results: Good correlation between direct and functional measurement of apixaban could be found. The Passing-Bablok regression analysis revealed an intercept of $3.90 \mu \mathrm{g} / \mathrm{L}$ (95\% confidential interval (95\% CI), 1.26 to $7.29 \mu \mathrm{g} / \mathrm{L}$ ) and a slope of $0.93 \mu \mathrm{g} / \mathrm{L}$ (95\% CI, 0.84 to $0.98 \mu \mathrm{g} / \mathrm{L}$ ). The correlation coefficient was 0.97 . Good correlation was also found for dabigatran comparing our online LC-MS/MS assay and the functional assay from Siemens INNOVANCE DTI performed automatically on a Sysmex CS-5100 system from Siemens Healthcare Diagnostics GmbH. The correlation coefficient was 0.98. The Passing-Bablok regression analysis of the dabigatran measurements revealed an intercept of 2.76 $\mu \mathrm{g} / \mathrm{L}$ ( $95 \%$ CI, -1.15 to $6.78 \mu \mathrm{g} / \mathrm{L}$ ) and a slope of $0.85 \mu \mathrm{g} / \mathrm{L}$ ( $95 \%$ CI, 0.76 to $0.95 \mu \mathrm{g} / \mathrm{L}$ ). No regression analysis was performed for edoxaban measurements because of the small sample size. However, it can be calculated from the measured values that the concentration measured by mass spectrometry was generally lower than measured by the functional assay. There was a good correlation between the rivaroxaban measurements performed by our online SPE-UPLC-MS/MS method and these drug measurements in patient's samples using the INNOVANCE Heparin assay from Siemens Healthcare Diagnostics GmbH performed automatically on a Sysmex CS-5100 system. The correlation coefficient was 0.98. The Passing-Bablok regression analysis of our online LC-MS/MS assay and the functional assay from CoaChrom Diagnostica GmbH performed automatically on a Sysmex CS-5100 system revealed an intercept of $6.90 \mu \mathrm{g} / \mathrm{L}$ (95\% CI, 2.21 to $20.9 \mu \mathrm{g} / \mathrm{L}$ ) and a slope of $0.88 \mu \mathrm{g} / \mathrm{L}$ (95\% CI, 0.81 to $0.95 \mu \mathrm{g} / \mathrm{L}$ ).

Conclusion: In summary, good correlation could be found between our online SPE-UPLC-MS/MS method and the used commercially available coagulation assays. However, direct measurement of DOACs may be required in some clinical circumstances.

\section{DGKL-P077}

\section{Mass spectrometry based therapeutic drug monitoring of Biologicals}

\author{
Nina Scheffe ${ }^{1}$; Marvin Müller ${ }^{1}$; Peter Findeisen ${ }^{2}$; Rupert Schreiner ${ }^{1}$ \\ ${ }^{1}$ MVZ Labor Limbach Heidelberg, Entwicklung, Heidelberg, Germany; ${ }^{2}$ MVZ Labor Limbach Heidelberg, Studien, Heidelberg
}

Introduction: Biologicals are a heterogeneous group of drugs that are produced by living organisms using genetic or biological technology. These comprise a constantly growing group of monoclonal antibodies (mAbs), fragments of antibodies and fusion proteins. Specifically, mAbs are utilized for treatment of several diseases including cancer, inflammation, allergies, autoimmune- and infectious diseases. Inadequate $\mathrm{mAb}$ concentration in serum of patients is associated with subsequent loss of response. Hence, Therapeutic drug monitoring (TDM) of mAbs has the potential to lead to more effective individual dosing.

Materials and Methods: A mass spectrometry based workflow (LC-MS/MS) was established for the quantification of therapeutic mAbs (e.g. Infliximab, Adalimumab, Vedolizumab, Golimumab) in serum. Commercial peptides were used as internal standards. Several validation parameters including dynamic range, LOD (lower limit of detection) and LOQ (lower limit of quantification), selectivity and reproducibility were evaluated. Results were compared to commercially available ELISA assays.

Results: LC-MS based quantification of mAbs from clinical specimens is fit for purpose as test characteristics (LOD, LOQ, linearity and CV) are comparable to those of respective ELISA-kits. However, the quantification of mAbs with ELISA-assays is interfered with the presence of antidrug antibodies (ADA). In contrast, our MS-based workflow was not influenced by the presence of ADAs and lead to more conclusive results. 
Conclusion / Discussion: TDM of mAbs may support dose decision to avoid unnecessary switch in medication or possible overtreatment. However, results from ELISA assays might be difficult for interpretation as interference due to ADAs does affect results. MS-based assays might be helpful for standardization of different assay-platforms for TDM of Biologicals.

\section{DGKL-P078}

\section{4/7 Drugs of Abuse Screening}

Frank Streit'; Gry Helene Dihazi²; Marcel Grapp ${ }^{3}$; Lutz Binder ${ }^{2}$

${ }^{1} U M G L$ UMG Goettingen, UMG-Labor, Goettingen, Germany; ${ }^{2} U M G L$ UMG Goettingen, Institut für klinische Chemie / UMG-Labor, Goettingen, Germany; ${ }^{3} U M G L$ UMG Goettingen, Rechtsmedizinisches Labor für forensisch-toxikologische Analytik und klinisch toxikologisches Labor, Goettingen, Germany

Background: A broad spectrum of LC-MS/MS methods for therapeutic drug monitoring is currently commercially available, e.g. for anticonvulsive drugs, neuroleptic drugs, benzodiazepines, tricyclic antidepressants as well as for selective serotonin reuptake inhibitors (SSRI's). Most of these drugs are also relevant for clinical toxicological screenings. Screening immunoassays using polyclonal antibodies are still mostly used in emergency labs despite possible false positive and false negative results. Until now, the analysis of these drugs with mass spectrometric methods is restricted to special skilled staff and therefore not available in most emergency laboratories 24/7 routine analysis.

Aim: The aim of this study was to establish an alternative cost effective standardized methodology for screening up to 150 common used drugs. We validated a 97-drug-method containing 12 tricyclic antidepressant drugs, 26 different anticonvulsive drugs, 35 benzodiazepines, 11 neuroleptic drugs as well as 13 SSRI's in one single 7 min semi-quantitative screening method.

Method: We used the fully automated LC-MS/MS-system equipped with an automated sample preparation module (CLAM-2000, Shimadzu) to evaluate the possibility of a $24 / 7$ drug screening performed by technicians without chromatographic or mass spectrometric experience. Calibration frequency was reduced from daily to weekly and quality controls were measured twice a day. Internal standards, precipitation solution, calibrators and controls were loaded daily into the CLAM-2000 trays. Patient plasma samples were placed on the device during the day. The CLAM-2000 unit automatically performed the plasma protein precipitation and filtration separation before the filtrate was transferred to the LC for further analysis of the given drugs. Chromatographic separation of the metabolites was performed with a linear gradient at $45^{\circ} \mathrm{C}$. The target compounds were identified by parent ions and optimized MRM transitions.

Results: We successfully developed a LC-MS/MS method to detect and quantitate more than 100 different drugs of abuse in one single run. 100 patient samples were measured and the most abundant drugs were automatically identified. All compounds, which were identified with our routine forensic toxicological LC-QTOF screening methods, were also detected in the novel LC-MS/MS screening method.

Conclusion: The simultaneous measurement of more than 100 drugs within the same method makes CLAM-2000 a valuable device for 24/7 use in clinical diagnostics. Above all, the robustness and the excellent precision and accuracy that we confirmed in our evaluation study were convincing. In addition, the similarity of the user-friendly control panel of the CLAM-2000 to the common clinical chemistry analyzers makes LCMS analysis easy to use in emergency labs.

\section{DGKL-P079}

\section{Pharmacokinetics of piperacillin in critically ill patients: a population subset-analysis of the TARGET-study}

Carsten Müller ${ }^{1}$; Niels Winkelmann²; Torben Semer ${ }^{3}$; Thomas Streichert ${ }^{4}$; Martin H. J. Wiesen Wiesen ${ }^{5}$; Guido Michels ${ }^{6}$; Andreas Hohn ${ }^{7}$;

Thorsten Annecke

${ }^{1}$ Universitätsklinikum Köln, Zentrum für Pharmakologie - TDM, Köln, Germany

${ }^{2}$ University Hospital of Cologne, Centre of Pharmacology, Cologne, Germany, Therapeutic Drug Monitoring,, Köln,Germany; ${ }^{3}$ University Hospital

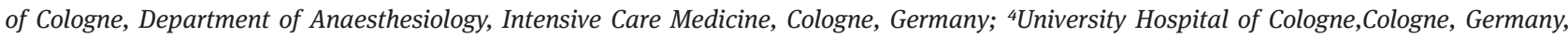
Department of Clinical Chemistry, Köln,Germany; ${ }^{5}$ University Hospital of Cologne, Centre of Pharmacology, Therapeutic Drug Monitoring, Cologne, Germany, Therapeutic Drug Monitoring, Köln, Germany; 'University Hospital of Cologne, Intensive Care Medicine, Cologne, Germany, Department III of Internal Medicine, Köln,Germany; ${ }^{7}$ University Hospital of Cologne, Intensive Care Medicine, Cologne, Germany, Department of Anaesthesiology, Köln,Germany

Background: Piperacillin in combination with tazobactam is indicated for the treatment of serious, hospital acquired-infections on intensive care units (ICU). In a prospective, multi-center, single-blinded, randomized, controlled, treatment optimization study (TARGET-study) the pharmacokinetics of a population subset (study-center Cologne) of piperacillin were investigated in two groups: Therapeutic Drug Monitoring-group (TDM-group, age: 54.3 - 88.9 years, range) $(\mathrm{n}=13)$ and Control-group (Control, age: 33.4 - 78.5 years, range) $(\mathrm{n}=13)$. The recommended dosage regimen consisted in a bolus application of $4 \mathrm{~g}$ piperacillin and continuous infusion of $12 \mathrm{~g} / 24 \mathrm{~h}(6.3 \mathrm{ml} / \mathrm{h})(\mathrm{eGFR} \geq 20 \mathrm{ml} / \mathrm{min})$. Blood plasma samples were collected once daily during continuous infusion after achievement of steady-state levels. 
Methods: Piperacillin was measured in blood plasma by validated HESI-LC-MS-MS-method. The following pharmacokinetic parameters: $\mathrm{t} 1 / 2 \alpha, \mathrm{t} 1 / 2 \beta$ and $\mathrm{Vd}$, as well as average daily application rate (DAR) of piperacillin were calculated in each study arm using a two compartment model for the disposition of piperacillin using MWPharm ++ .

Results:

\begin{tabular}{|c|c|c|c|c|c|}
\hline Study-arm & $\mathbf{N}$ & $\mathrm{t} 1 / 2 \alpha[\mathrm{h}] \pm(\mathrm{SD})$ & $\mathrm{t} 1 / 2 \beta[\mathrm{h}] \pm(\mathrm{SD})$ & DAR $[\mathrm{mg}] \pm(\mathrm{SD})$ & $\operatorname{Vd} \pm(S D) *$ \\
\hline TDM-group & 13 & $1.11 \pm 0.14$ & $9.48 \pm 4.15$ & $12653 \pm 2649$ & $29.94 \pm 13.93$ \\
\hline Control-group & 13 & $1.16 \pm 0.06$ & $12.50 \pm 5.12$ & $12870 \pm 435$ & $43.38 \pm 17.99$ \\
\hline
\end{tabular}

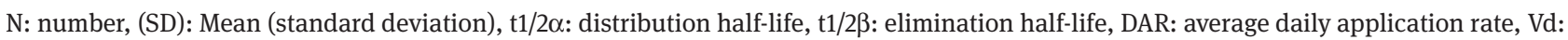
volume of distribution; * Statistically significant difference between TDM- and control-group (T-Test, $\mathrm{p}<0.05$ )

Mean elimination half-lifes $(\mathrm{t} 1 / 2 \alpha, \mathrm{t} 1 / 2 \beta)$ and average daily application rate revealed no significant difference between the two groups. In contrast, calculated difference in Vd between TDM-group and Control-group is statistically significant. as a consequence of age, disease-driven variation in pharmacokinetics and hydrophilic physicochemical properties of piperacillin.

Conclusion: Within a subpopulation of the TARGET-Study preliminary data with a focus on pharmacokinetic analysis are presented. Calculated Vd of both groups was distinctly larger in both groups compared to healthy volunteers (Vd $14 \mathrm{~L})$. The difference within TDM-group and Control-group was statistically significant in part as a result of disease-driven variation in pharmacokinetics and hydrophilic physicochemical properties of piperacillin. Although only a small population of severely ill patients on ICU was presented, pharmacokinetic data corresponds to preliminary already published data.

\section{DGKL-P080}

\section{Isotope dilution TurboFlow ${ }^{\mathrm{TM}}$ LC-HRMS for the simultaneous quantification of 12 antimycotics in human serum}

Carina Schuster; Michael Paal; Johanna Lindner; Michael Vogeser

Klinikum der Universität München, Institut für Laboratoriumsmedizin, München, Germany

Objective: Invasive fungal infections are a leading cause of morbidity and mortality in patients with compromised immunity. As critically ill patients have substantial pathophysiological abnormalities in relation to healthy volunteers they are at imminent risk failing antimicrobial pharmacokinetic/pharmacodynamic (PK/PD) target attainment. The aim of this study was to develop and validate an isotope-dilutionTurboFlow ${ }^{\mathrm{TM}}$-high resolution mass spectrometry (MS) method for therapeutic drug monitoring of the 12 most widely used systemic antimycotics. Method: Protein precipitation was followed by automated online sample clean-up with a Thermo Scientific ${ }^{\mathrm{TM}}$ Cyclone MCX-2 TurboFlow $^{\mathrm{TM}}$ column $(0.5 \times 50 \mathrm{~mm})$. With this column holding both anionic exchange and reversed-phase properties we were able to perform cleaning and focusing of analytes in a single step. After backflushing the TurboFlow ${ }^{\mathrm{TM}}$ column, analytic separation was performed on a Thermo Scientific $^{\text {TM }}$ Hypersil ${ }^{\text {TM }}$ GOLD C18 column $(50$ x $2.1 \mathrm{~mm}, 1.9 \mu \mathrm{m})$ using an acetonitrile/water gradient. Total run time was 4 minutes. 12 antimycotics, namely 5-flucytosine, amphotericin B, anidulafungin, caspofungin, fluconazole, isavuconazole, itraconazole, ketoconazole, micafungin, $\mathrm{OH}$ itraconazole, posaconazole and voriconazole were finally quantified in full MS scan mode in positive electrospray ionization (ESI + ) mode on a Thermo Scientific ${ }^{\mathrm{TM}} \mathrm{Q}$ Exactive $^{\mathrm{TM}}$ Focus Orbitrap ${ }^{\mathrm{TM}}$ high-resolution, accurate-mass spectrometer. Calibrators, QC samples, most internal standards and precipitation reagent from a commercial kit were used.

Results: Within a measuring range covering sub-therapeutic, therapeutic and toxic levels we observed good linearity ( $\mathrm{r} 2>0.995)$ and $\mathrm{S} / \mathrm{N}>200$ for lowest calibrator levels. Application of "double mode" TurboFlow ${ }^{\mathrm{TM}}$ enabled purification, concentration and quantification of a heterogeneous panel of antimycotic drugs from 129 to $1270 \mathrm{~m} / \mathrm{z}$.

Summary: With a short turn-around time the proposed method provides a simple and robust sample clean-up minimizing the interference of the sample matrix. Application of commercially available kit components allows for handling high level of convenience.

\section{DGKL-P081}

\section{Characterization of uracil catabolism variability in healthy volunteers and cancer patients}

Dominic Schärer'; Dominic Kummer $^{2}$; Christos Nakas ${ }^{3}$; Stefano Fontana ${ }^{4}$; Markus Jörger ${ }^{5}$; Ursula Amstutz² Carlo Largiadèr $^{2}$ ${ }^{1}$ Inselspital, Universitätsspital Bern, Universitätsinstitut für klinische Chemie, Bern, Switzerland; ${ }^{2}$ Inselspital, Bern University Hospital, and University of Bern, Institute of Clinical Chemistry, Bern, Switzerland; ${ }^{3}$ University of Thessaly, Department of Agriculture Crop Production and Rural Environment, Nea Ionia, Grece; ${ }^{4}$ Regional Blood Transfusion Service of the Swiss Red Cross, Bern, Switzerland; ${ }^{5}$ Cantonal Hospital St. Gallen, Department of Medical Oncology and Hematology, St. Gallen,Switzerland

The catabolic pathway of uracil (U) mainly determines the pharmacokinetics of the anticancer drug 5-fluorouracil (5-FU). Decreased activity of dihydropyrimidine dehydrogenase (DPD), the first catabolizing enzyme, was identified as a major predictor of 5-FU toxicity. However, 
phenotypic variability in the catabolism downstream of DPD by dihydropyrimidinase (DHP) and $\beta$-ureidopropionase (bUP) and its potential contribution to 5-FU toxicity has only partially been investigated so far. Here, we aimed to characterize the variability of metabolites and metabolic ratios of uracil catabolism and to evaluate their association with genetic variation in the DHP and bUP genes (DPYS and UPB1). Plasma concentrations of $U$ and its metabolites were determined by UHPLC-MS/MS and three variants in DPYS (c.1-1T >C and c.265-58T > C) and UPB1 (c.1-80C $>$ G) previously associated with 5-FU toxicity were genotyped in 320 healthy volunteers. Similarly, endogenous and 5-FU metabolite plasma concentrations were measured by UHPLC-MS/MS in 27 cancer patients. In healthy volunteers, sex-dependent differences were observed along the entire uracil catabolism pathway. In particular, we observed lower concentrations of all metabolites $(\mathrm{P} \leq 0.020)$ as well as lower $\beta$-ureidopropionic acid/dihydrouracil ratios (UPA/UH2; $\mathrm{P}=0.001$ ) in women, suggesting that reduced catabolism may contribute to higher 5-FU toxicity rates in females. In cancer patients, BAL/UPA ratios were altered during 5-FU infusion (after $2 \mathrm{~h}, \mathrm{P}<0.001$ ), indicating that bUP, besides DPD, may also play a rate-limiting role in uracil catabolism, especially in the progress of 5-FU-degradation, as bUP appears to be saturated based on the increasing FBAL plasma concentrations after $2 \mathrm{~h}$ of 5 -FU infusion $(\mathrm{P}<0.001)$.

\title{
Posterwalk Therapeutisches Drug Monitoring und Pharmakogenetik II
}

\section{DGKL-P082}

\author{
The importance of therapeutic drug monitoring (TDM) within colorectal cancer therapy with 5-Fluoruracil (5-FU) and \\ preanalytical aspects
}

Sonani Mindt ${ }^{1}$; Sihem Aida ${ }^{2}$; Michael Neumaier ${ }^{2}$; Ralf Hofhein $z^{3}$

${ }^{1}$ Universitätsmedizin Mannheim, Institut für Klinische Chemie, Mannheim, Germany; ${ }^{2}$ Mannheim Medical Faculty of Heidelberg University, University Hospital Mannheim, Institute for Clinical Chemistry, Mannheim, Germany; ${ }^{3}$ Mannheim Medical Faculty of Heidelberg University, University Hospital Mannheim, Day Treatment Center (TTZ), Interdisciplinary Tumor Center Mannheim (ITM) \& III Medical Clinic, Mannheim, Germany

Background: In Germany, about 25.000 patients with colorectal cancer die from their disease per year. Virtually all patients receive the 5-Fluorouracil (5-FU) as backbone for the medical treatment. The pharmacological effect of 5-FU is influenced by genetic polymorphisms of DPD (among others) as well as different dosage schemes. In order to personalize and optimize 5-FU treatment and to prevent toxic effects of the medication, area under the curve (AUC) can be estimated to follow for, individual drug adjustment. Currently, standard dosing of 5-FU chemotherapy is carried out according to body-surface area (BSA). The aim of the current study is to address one preanalytical aspect. Specifically, it is unclear if blood could be drawn at the side where the port system and the portable-pump system administering 5-FU are placed. Material/Methods: We collected EDTA blood samples of patients with gastrointestinal cancers over four month. These samples were collected from different sampling points (right vs. left cubital vein at the same time-point). 5-FU concentrations were measured in steady-state equilibrium based on nanoparticle immunoassay (My5-FU; Saladax Biomedical) and the AUC values obtained at different side were calculated. Results: A total of 40 patients consented to take part in this study. The measured 5-FU AUC had a median of 23.28 for the right arm (range 5.79-59.37) and a median of 23.36 for the left arm (range 5.32-61.03). AUC values showed no significant difference between right compared to left $\operatorname{arm}(\mathrm{p}<0.05)$.

Discussion: For an optimal treatment of the patients the AUC should be between 20 and 30 [mg x h x l]. Based on our results, $53 \%$ of the measured patients did not reach the target 5-FU concentration window. 37\% of the study population was underdosed (AUC 5.79-18.34) and 16\% of the patients with an AUC between 30.93 and 55.73 are even overdosed. According to these results we can demonstrate that treatment schemes with 5-FU which based on dosing according to BSA is unsatisfactory to achieve a drug concentration in the therapeutic range. An influence of the location of the venepuncture of the 5-FU concentration in plasma could not be verified.

Conclusion: The current pilot study shows that TDM should be regarded a useful way to make a significant contribution to optimize 5-FU treatment. The sampling point for blood collection has no effect on the measured 5-FU concentration in blood.

\section{DGKL-P083}

\section{The clinical benefit of pharmacogenetic testing to prevent adverse drug reactions - demonstrated by two case reports}

Mathis Römer'; Laura Mirbach $^{1}$; Angelika Duda ${ }^{1}$; Romy Eichner ${ }^{1}$; Michael Neumaier ${ }^{1}$; Verena Haselmann ${ }^{2}$

${ }^{1}$ Universitätsmedizin Mannheim, Institut für Klinische Chemie, Mannheim, Germany; ${ }^{2}$ Uniklinikum Mannheim, IKC, Mannheim, Germany

BACKGROUND: Nowadays, we claim to perform "P4 medicine" that is intended to be predictive, preventive, personalized and participatory. This also includes a personalized choice of treatment based on an individual's genetic profile to achieve the best therapeutic outcome and reduce serious adverse drug reactions (ADRs). However, ADRs are a leading cause of morbidity and mortality: approximately $5.7 \%$ of hospitalizations are due to ADRs in Germany causing estimated costs of 400 Million $€$ per year, $1 \%$ of ADRs being fatal with over $60 \%$ of these being evitable. Therefore, the Food and Drug Administration e.g. provides an overview of all pharmacogenomics relevant biomarkers with clear 
instructions for testing and dosage. In Germany, comparable recommendations are provided with medication package inserts or in specialist information, and are also available via VFA (Verband forschender Arzneimittelhersteller) and PharmGKB (Pharmacogenomics Knowledgebase). Nevertheless, in clinical practice pharmacogenomics tests are usually carried out when ADRs already occured.

METHODS: Two recent case reports from our University hospital are illustrated highlighting the problems associated with ADRs depending on the individual's genetic profile. Genetic testing is carried out as part of patient's standard care using pyrosequencing for mutational analysis. RESULTS: Patient A presented with a primary CNS lymphoma (PCNSL). Under treatment with high-dose methotrexate (MTX) followed by leucovorin rescue, he developed an acute renale failure (ARF) 24 hours after application. A genetic testing carried out at this time point identified a compound-heterozygosity for MTHFR C677T and A1298C that is associated with reduced enzyme activity and MTX toxicity. Administration of carboxypeptidase-G2 (CPG2) was finally sufficient to decrease MTX concentration and normalization of renal function. Patient B had a medical history of a newly diagnosed rectal adenocarcinoma (T3 N1b M0) with a recently started neoadjuvant radiochemotherapy with Capecitabine (prodrug of 5-Fluouracil). The patient presented with severe nausea, vomiting, and diarrhea 14 days after treatment initiation. Laboratory testing revealed a leukopenia and thrombocytopenia that were progressive within the next five days finally causing the death of the patient. Genetic testing was carried out at day of presentation of ADRs and revealed a heterozygous DPYD Exon 14 skipping mutation. CONCLUSION: The two case reports provide excellent examples for evitable ADRs causing severe or even fatal side effects. This highlights that personalized medicine is not part of standard clinical care at the moment in Germany. Hopefully, the awareness of the clinical and economic importance of this will help to improve the outcome of patients in future.

\section{DGKL-P084}

\section{Quantification of nimodipine concentrations in plasma and cerebrospinal fluid of patients following aneurysmal subarachnoid hemorrhage}

Sonani Mindt ${ }^{1}$; Ursala Tokhi²; Daniel Hänggi²; Hendrik Bracht ${ }^{3}$; Michael Neumaier ${ }^{1}$; Hans-Jürgen Groß ${ }^{3}$

${ }^{1}$ Universitätsmedizin Mannheim, Institut für Klinische Chemie, Mannheim, Germany; '2University Hospital Mannheim, Mannheim, Germany, Department of Neurosurgery, Mannheim, Germany; ${ }^{3}$ University Hospital Ulm, Ulm, Germany, Department of Intensive Care Medicine, Ulm, Germany; ${ }^{3}$ University Hospital Ulm, Ulm, Germany, Institute for Clinical Chemistry, Ulm

Objective: Delayed cerebral ischemia (DCI) is an important cause of morbidity after aneurysmal subarachnoid hemorrhage (aSAH). Its time of appearance is uncertain, and its complications are devastating affecting patients' outcome. Nimodipine is the only drug approved by the FDA for improving outcome after SAH. Clinically, however, there are no specific values of this drug in plasma or cerebrospinal fluid (CSF) during aSAH treatment that could be associated to outcome improvement. Our aim was to establish a method to measure nimodipine concentrations in plasma and CSF of patients after aSAH, and to determine the therapeutic concentrations of this drug associated with clinical response.

Methods: From June 2017 to July 2018, we prospectively collected clinical data of patients with aSAH admitted to our neurosurgical department. All the included patients received nimodipine orally, and followed a standard dose of 60 mg every 4 hours. Patients, who developed clinical vasospasm during their in-hospital stay, underwent intra-arterial application of nimodipine (800 ng/ml), followed by angiographic control. In order to determine nimodipine concentrations, we established a method using the high performance liquid chromatography (LC) coupled with mass spectrometric (MS) analysis (LC/MS) for further quantification of nimodipine concentrations in serum and in CSF.

Results: Our LC/MS method allowed quantification of nimodipine concentrations in plasma with lower limit of quantification (LOQ) of 0.2 ng/ $\mathrm{ml}$, and with an LOQ of $0.15 \mathrm{ng} / \mathrm{ml}$ in CSF respectively. The MS/MS ion transitions were 419.2 $\square 343.0$ for nimodipine and $426.0 \square 350.0$ for the internal standard. Nimodipine concentrations in CSF were significantly lower (MW: $0.35 \mathrm{ng} / \mathrm{ml}$ ) than plasma concentrations (MW: $5.76 \mathrm{ng} /$ $\mathrm{ml}$ ). After intra-arterial nimodipine boluses for treatment of vasospasms, serum and CSF nimodipine concentrations were slightly higher than patients who received only oral nimodipine.

Conclusion: The LC/MS analysis allows identification and measurement of nimodipine concentrations in plasma and CSF of patients after aSAH. The initial stage of this study provided the first concentrations values of nimodipine in plasma and CSF. Furthermore, we demonstrated that intra-arterial boluses of nimodipine had higher concentrations in plasma and CSF, when compared to the oral application

\section{DGKL-P085}

\section{4/7 Monitoring of Antibiotic Drugs}

Frank Streit'; Gry Helene Dihazi²; Ivana Markovic²; Tatjana Khromov²; Catharina Cohrs²; Marcel Grapp'; Lutz Binder ${ }^{2}$

${ }^{1} U M G L$ UMG Goettingen, UMG-Labor, Goettingen, Germany; ${ }^{2} U M G L$ UMG Goettingen, Institut für klinische Chemie / UMG-Labor, Goettingen, Germany; ${ }^{3} U M G$ Goettingen, Rechtsmedizinisches Labor für forensisch-toxikologische Analytik und klinisch toxikologisches Labor, Goettingen, Germany

Background: Therapeutic drug monitoring (TDM) and dosage individualization is becoming increasingly important, particularly in critical clinical situations with disturbed, insufficient correlation between drug dosage and pharmacodynamics. In septicemia patients, antibiotic 
treatment with intermittent bolus administration is increasingly replaced by prolonged or continuous infusion. This allows a better interpretation of antibiotic plasma concentrations and a rational TDM-based dose adjustment with respect to therapeutic efficacy. Therefore, clinicians are calling for 24/7 measurement of antibiotic concentrations. Liquid chromatography with tandem mass spectrometry (LC-MS/MS) is an appropriate system for simultaneous measurement of multiple drugs. Until today, however, technical preconditions for LC-MS/MS use in emergency labs were not given.

Aim: The aim of the present study was to develop a multiple drug monitoring method for 11 (12) frequently used, important antibiotics and to make these measurements available day and night, including weekends. The method should comprise meropenem, piperacillin/tazobactam, cefotaxime, ceftazidime, linezolid, cefuroxime, ampicillin, colistin, ciprofloxacin, free and total concentrations of flucloxacillin and ceftriaxone. Method: Measurements were performed on a LCMS-8050 system (Shimadzu) including a CLAM-2000 automated sample preparation module. Stabilized internal standards, precipitation solution, and controls were reloaded daily into the CLAM-2000 system. According to calibrator stability, calibration frequency was reduced from daily to weekly and quality controls were checked twice a day. Patient plasma samples were placed on the device according to their arrival time in the lab. The CLAM-2000 unit automatically performed sample protein precipitation and separation by filtration, before the filtrate was transferred to the autosampler. Chromatographic separation of the compounds was performed with a sharp linear gradient on a UPLC column (Waters) at $45^{\circ} \mathrm{C}$. Target compounds were identified using optimized MRM transitions. Quantification was done with deuterated internal standards.

Results: Inaccuracy of quality controls within one week was below 10\% CV. The imprecision of patient samples and controls was also less than $10 \%$ CV. Stability of the system was demonstrated by a constant signal of the internal standard with less than 10\% inaccuracy. Repeated measurements of controls kept on the instrument for 24 hours showed an inaccuracy of less than $5 \%$. The deviation of repeated measurements from non-stabilized patient samples was below $5 \%$ within 12 hours.

Conclusion: The simultaneous measurement of 11 (12) different antibiotics with a single method makes the CLAM-2000 an attractive device for 24/7 use in clinical diagnostics. The automated, easy-to-use system allows technical assistants even in the emergency lab to operate the system after a limited initial training period.

\title{
DGKL-P086
}

\section{Unexpected Codeine findings in patients with Morphine (retard) substitution}

\author{
Julietta Ursula Schweiger ${ }^{1}$; Svantje Hebenbrock ${ }^{1}$; Jan Kramer' ${ }^{2}$ Lars Wilhelm ${ }^{1}$ \\ ${ }^{1}$ LADR GmbH •Ihr Labor vor Ort!, Toxikologie, Geesthacht, Germany; ${ }^{2}$ LADR GmbH • Ihr Labor vor Ort!, Ärztliche Leitung \& Geschäftsführung, \\ Geesthacht,Germany
}

Background: Morphine (retard) is a drug used since 2015 for substitution of opioid addicted patients as part of a medical and psychosocial program. To detected additional abuse of heroin or morphine it is useful to analyze urine, blood or saliva with Liquid chromatography-mass spectrometry (LCMS). Besides heroine markers also codeine as an alkaloid in poppy seeds as well as hydromorphone as a minor metabolite of morphine were detected. Aim of this study was to find a cut-off for codeine concentrations in urine samples to distinguish morphine (retard) intake from heroine abuse, with a statistical evaluation of about 1000 samples.

Material \& Methods: About 1000 LCMS data from urine of patients in an opioid replacement therapy were evaluated for the concentration of morphine, codeine and hydromorphone. Furthermore the medication and results for heroine marker were acquired. Statistical analysis was performed by anonymous evaluation. Boxplots, means and quantiles were done by using the statistical program R.

Results: For codeine the interquartile range of the boxplot was much smaller in the morphine (retard) group, than for the methadone group. We assumed that patients in the methadone group had additional abuse of morphine or heroine. The end of the upper whisker of the boxplot of the morphine (retard) group was lower, $176 \mathrm{ng} / \mathrm{ml}$, creatinine normalized, than the quantile 1 of the methadone group, $190 \mathrm{ng} / \mathrm{ml}$, creatinine normalized.

Discussion: The concentration of codeine in urine samples of patients substituted with morphine (retard) did not have a great variation compared against the concentration of codeine in urine samples of patients that took heroine or morphine abusive.

The end of the upper whisker of the boxplot of the morphine (retard) group was defined as limit / cut-off. It is highly probable that above this cut off, an additional abuse if heroin or morphine has occurred.

\section{DGKL-P087}

\section{4/7 Antiepileptic Drugs}

Gry Helene Dihazi ${ }^{1}$; Lutz Binder ${ }^{1}$; Marcel Grapp²; Umut Bayrak' ${ }^{1}$ Tatjana Khromov ${ }^{1}$; Ivana Markovic' ${ }^{1}$;rank Streit ${ }^{1}$ ${ }^{1} U M G$-Göttingen, Institut für Klinische Chemie / UMGL, Göttingen, Germany; ${ }^{2} U M G$-Göttingen, Rechtsmedizinisches Labor für forensischtoxikologische Analytik und klinisch-toxikologisches Labor, Göttingen, Germany

Background: A broad spectrum of antiepileptic drugs (AEDs) is meanwhile available for the treatment of epilepsy. In this group of neurological disorders, brain activity becomes abnormal, causing seizures or periods of unusual behavior, sensations, and sometimes loss 
of consciousness. Several factors (e.g. patient compliance, drug clearance) may influence plasma drug levels and lead to unpredictable efficacy or adverse drug effects. Depending on the kind of drug used, TDM of AEDs is frequently recommended. Thus, TDM for carbamazepine is strongly recommended due to a narrow therapeutic range and severe side effects in the case of overdosage. Liquid Chromatography with Tandem Mass Spectrometry (LC-MS/MS) represents an established analytical system for simultaneous measurement of multiple drugs (e.g. AEDs). However, LC-MS/MS systems lack features required for use under 24/7 conditions with frequently changing and less qualified staff.

Aim: The aim of this study was to develop and to implement a methodology for 26 different AEDs with a fully automated LC-MS/MS system equipped with an integrated sample preparation module (CLAM-2000, Shimadzu). Furthermore, we wanted to test the feasibility of a 24/7 monitoring service by technicians without previous chromatographic or mass spectrometric experience.

Method: The CLAM-2000 unit automatically performed plasma protein precipitation and filtration before the filtrate was transferred to the LC. Chromatographic separation of the analytes was performed with a sharp linear gradient on a fused core column at $45^{\circ} \mathrm{C}$. The target compounds were identified by parent ions and optimized MRM transitions. Quantification was performed by using deuterated internal standards. Calibration stability allowed a reduction of calibration frequency from daily to weekly. Quality controls were checked twice a day. Internal standards, precipitation solution, and controls were loaded daily into the CLAM-2000 trays. Patient plasma samples were placed on the device after their arrival in the lab.

Results: The inaccuracy of the internal standards was less than $10 \% \mathrm{CV}$ within one week, which demonstrates the stability of the system. Imprecision and inaccuracy of quality controls and patient samples was below $10 \% \mathrm{CV}$. Repeated measurements of controls kept on the instrument for $24 \mathrm{~h}$ showed an inaccuracy of less than 5\%. The deviation of repeated measurements of patient samples was below $5 \%$ within $12 \mathrm{~h}$.

Conclusion: Simultaneous measurement of 26 different AEDs makes CLAM-2000 an attractive device for 24/7 use in clinical and toxicological diagnostics. Robustness, excellent precision, and accuracy, which were confirmed in our evaluation study, were convincing. In addition, the user-friendly control panel and familiar user interface of the CLAM-2000 makes LC-MS valuable for 24/7 emergency analyses. For future routine diagnostics, bi-directional communication and shorter electronic response times are desired and are meanwhile under development.

\title{
DGKL-P088
}

\section{Validation of a LC-MS/MS based immunosuppressive drug TDM assay - a hybrid between lab developed procedures and industrial test design}

\author{
Bernadette Näscher ${ }^{1}$; Christian Timm ${ }^{\text {; }}$ Dagmar Siefert ${ }^{2}$; Maria Shipkova ${ }^{3}$; Christoph Seger ${ }^{1}$ \\ ${ }^{1}$ Risch Laboratory Group, ---, Buchs, Switzerland; 'Klinikum Stuttgart, Zentralinstitut für Klinische Chemie und Laboratoriumsmedizin, Stuttgart, \\ Germany; ${ }^{3}$ SYNLAB Holding Deutschland, MVZ Leinfelden-Echterdingen GmbH, Leinfelden-Echterdingen, Germany
}

Objective: Modern LC-MS/MS based immunosuppressant drug TDM (ISD-TDM) relies on one- or two-dimensional liquid chromatography (LC). Commercial IVD-CE certified assay realizations which come fully validated are based on LC settings (e.g. properties of the stationary and mobile phase etc.) undisclosed to the applicator. Since LC is an analytical science with long tradition and very well understood mechanisms of action, the transferability of a separation setup for a given set of analytes is feasible. This hold especially true if no high-resolution LC separation is needed - as it is the case for ISD-TDM with MS/MS detection. Hence, adding whole-blood ISD-TDM to a serum / plasma based TDM assay panel realization already in place seems feasible.

Methods: ISD-TDM establishment was based on an installation of the IVD-CE certified commercial ClinMass ${ }^{\circledR}$ TDM Kit System (Recipe, Munich, Germany) already operating in our laboratory. The isocratic mobile phase elution regimen of the LC separation dimension in the online-SPE-LC-MS/MS ISD-TDM kit of the same vendor was applied to the stationary phase of this assay. SRM transitions for the analytes cyclosporine A, tacrolimus, sirolimus, and everolimus (CIC, TAC, SIR, EVE, respectively) as well as the sample preparation procedure (with minor adaption) were transferred from the ISD assay. Hence a "hybrid” LC-MS/MS assay was generated and further optimized for decent signal generation prior to validation under calibration with commercial calibrator materials (Recipe, Munich, Germany).

Results: The realized LC-MS/MS assay was found to provide results which were precise (inter-assay CV of QC: $2.7-7.0 \%$ ) and true (90\% of 166 tested proficiency testing samples within $\pm 15 \%$ of target). No matrix effects were observed, interference testing with several dozen drugs did not show any signs of additional signals. Sufficient sensitivity (LLOQ CIC $10 \mu \mathrm{g} / \mathrm{l}$, TAC, SIR, EVE $0.5 \mu \mathrm{g} / \mathrm{l}$ ) guaranteed quantitative analysis of clinical samples. A platform comparison study (120 randomly chosen anonymized residual patient samples from a clinical environment) showed sufficiently good results with only minor absolute mean deviations which are clinical insignificant (CIC 6.5 $\mu \mathrm{g} / \mathrm{l}$, TAC, SIR, $\mathrm{EVE}< \pm 0.6 \mu \mathrm{g} / \mathrm{l})$.

Conclusion: Traditionally, ISD-TDM with the need of working with whole blood specimen is seen isolated from other TDM assays, e.g. for psychoactive drugs. With our approach we did prove, that this very artificial barrier can be overcome and ISD-TDM can be with ease embedded into the setting of a multi-assay TDM platform devoted to offer a daily measurement service for dozens of analytes.

Disclaimer: The presented lab developed test must be considered a research use only (RUO) application. This presentation must not be mistaken as performance evaluation study in accordance with the current IVD regulations (IVDR 2017/746). 


\section{DGKL-P089}

\section{Evaluation of DOAC measurement with the reagents INNOCANCE® Heparin and INNOVANCE® DTI on the Sysmex CS-5100 coagulation analyzer}

Tobias Flieder; Franziska Knüttgen; Katharina von Bargen; Joachim Kuhn; Andreas Hammerschmidt; Cornelius Knabbe; Ingvild Birschmann Herz- und Diabeteszentrum Nordrhein-Westfalen, Institut für Laboratoriums- und Transfusionsmedizin, Bad Oeynhausen, Germany

Introduction: Unlike warfarin, direct oral anticoagulants (DOACs) do not need to be monitored through laboratory testing, and no dose adjustment is required. Due to the widespread use of the DOACs dabigatran, rivaroxaban, apixaban and edoxaban, as argatroban, however, there are increasingly situations in which a plasma level of the drug is preferable. Meanwhile, for measuring DOACs, some tests are available for routine devices, but require adjustments to the appropriate device, since they are third-party reagents. To reduce the number of reagents and thus the costs, the aim of our work was to measure all anti-Xa or anti-IIa substances with one reagent each. Therefore, we evaluated the measurement of DOACs on the routine coagulation analyzer CS-5100 with the appropriate reagents from Siemens Healthcare.

Methods: All measurements were performed on the Sysmex CS-5100 coagulation analyzer. The INNOVANCE® Heparin and the INNOVANCE ${ }^{\circ}$ DTI reagent from Siemens were used. Controls and calibrators were from Technoclone (apixaban, rivaroxaban), Coachrom (argatroban), STA (edoxaban) and Siemens Healthcare (dabigatran). Additionally, the DOAC concentrations were measured with LC-MS/MS. We evaluated the CV, LOD and recoveries for the assays. Patient samples were measured for a method comparison with LC-MS/MS, the previously used tests and the INNOVANCE® reagents. We also investigated the stability of the reagents for these applications.

Results: The CVs were between $1.1 \%$ and $4.7 \%$ and the LOD was $<10 \mathrm{ng} / \mathrm{ml}$ for the DOAC assays and $<0.005 \mu \mathrm{g} / \mathrm{ml}$ for the argatroban assay. The correlation in the measurement of patient samples on the CS-5100 and with the LC-MS/MS showed an $\mathrm{r}^{2}$ of $>0.95$. The recoveries had a mean deviation of $<15 \%$ and $<17 \mathrm{ng} / \mathrm{ml}$ or $0.03 \mu \mathrm{g} / \mathrm{ml}$, respectively. The reagents showed good measurement results of the control samples even if the on-board stability was exceeded.

Conclusion: For hospitals treating emergency patients, the ability to measure the residual concentration of DOACs becomes important as more and more patients undergo emergency treatment under this therapy.

If a laboratory uses a CS-5100, the INNOVANCE® Heparin and INNOVANCE® DTI reagents are fast and stable alternatives for DOAC and argatroban measurement using only one reagent for all FXa-inhibiting drugs and one for FIIa-inhibiting drugs.

\section{DGKL-P090}

\section{MONITORING OF LOW DABIGATRAN CONCENTRATIONS: DIAGNOSTIC ACCURACY AT CLINICAL RELEVANT DECISION THRESHOLDS}

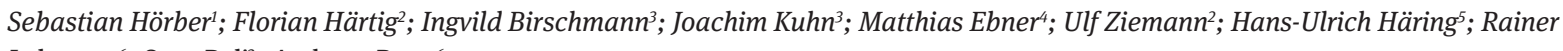
Lehmann ${ }^{6}$; Sven Poli²; Andreas Peter

${ }^{1}$ Universitätsklinikum Tübingen, Department of Internal Medicine, Division of Endocrinology, Diabetology, Vascular Medicine, Nephrology and Clinical Chemistry, Tübingen, Germany; ${ }^{2}$ Universitätsklinikum Tübingen, Neurologische Klinik, Tübingen, Germany; ${ }^{3}$ Herz- und Diabeteszentrum NRW, Universitätsklinik der Ruhr-Universität Bochum, Institut für Laboratoriums- und Transfusionsmedizin, Bad Oeynhausen, Germany; ${ }^{4}$ Charité-Universitätsmedizin Berlin, Kardiologische Klinik, Berlin, Germany; ${ }^{5}$ Universitätsklinikum Tübingen, Medizinische Klinik IV, Tübingen, Germany; ${ }^{6}$ Universitätsklinikum Tübingen, Medizinische Klinik IV - Zentrallabor, Tübingen, Germany

BACKGROUND: The direct oral anticoagulant dabigatran is used in the prevention of stroke and systemic embolism in patients with nonvalvular atrial fibrillation and for treatment of venous thromboembolism. Routine drug monitoring is not required for dabigatran, but measurements are gaining importance in emergency situations such as bleeding complications, thrombolytic therapy or urgent surgery. Current assays show a good performance at normal and high dabigatran concentrations but are limited at low concentrations. Therefore, special focus should be given to determination of low concentrations around the clinical decision threshold values of $30 \mathrm{and} 50 \mathrm{ng} / \mathrm{mL}$.

METHODS: Dabigatran concentrations of 293 plasma samples taken from 51 patients were measured with the Siemens INNOVANCE® direct thrombin inhibitor assay (DTI), the Biophen direct thrombin inhibitor assay (BDTI), the modified BDTI using a low range calibrator (BDTIlow), the Hemoclot direct thrombin inhibitor assay (HTI) and an Ecarin clotting time assay (ECT). All assay results were compared to the reference method, ultra-performance liquid chromatography-tandem mass spectrometry, and sensitivity, specificity and likelihood ratios were calculated for thresholds of 30 and $50 \mathrm{ng} / \mathrm{mL}$.

RESULTS: DTI, BDTI-low and ECT showed very strong correlation and high agreement with the reference method and improve determination of low dabigatran concentrations. ROC curve analyses revealed very high accuracy for DTI (AUC = 0.9891), BDTI-low (AUC = 0.9801) and ECT (AUC $=0.9959)$ measurements at the threshold of $30 \mathrm{ng} / \mathrm{mL}$. HTI (AUC $=0.941)$ and BDTI (AUC $=0.972)$ showed reduced accuracy at this threshold. Values for sensitivity and specificity were calculated for DTI (98/90\%), BDTI-low (87/95\%), ECT (97/96 \%), BDTI (99/82 \%) and HTI $(86 / 89 \%)$ measurements at the threshold of $30 \mathrm{ng} / \mathrm{mL}$. 
CONCLUSIONS: DTI, BDTI-low and ECT are able to measure low dabigatran plasma concentrations with very high and reliable accuracy for around the clock determination and are therefore suitable to guide treatment decisions in routine and emergency situations.

\title{
Posterwalk Analytische Qualität und Labormanagement I
}

\section{DGKL-P091 / PV12}

\author{
On-line Real Time Monitoring of Pre-analytical Hemolysis index - Potentials of a New IT Solution
}

\author{
Sebastian Wegerer'; Feodora Trifonova ${ }^{1}$; Wolf-Jochen Geilenkeuser ${ }^{2}$; Michael Neumaier' ${ }^{1}$; Karin Janetzko ${ }^{1}$ \\ ${ }^{1}$ Universitätsmedizin Mannheim, Institut für Klinische Chemie, Mannheim, Germany; ${ }^{2}$ Referenzinstitut für Bioanalytik, Labormedizin, Bonn, \\ Germany
}

\begin{abstract}
As an indicator for sample integrity the hemolysis index (HI) plays an important role in the quality control and management in a certified clinical laboratory. In order to facilitate accurate and swift controlling and thus adequate management of pre-analytical errors we propose here an automated system for internal monitoring of HI. Furthermore we describe a project of the Reference Institute for Bioanalytics, Germany for an online data base (Pre-analytical Benchmarking Database, PrBeDa), which to date deliberates over 1.5 million specimens from numerous nation-wide health facilities. The described Hemolysis Index Data Base allows relating hemolysis in specimens from hospital wards and functional units to data sets from respective units in the database. It supports the identification of causes for hemolysis by comparison of respective preanalytical impacts and accordingly facilitates troubleshooting. The current study describes for the first time an approach for instant, online quality assessment and its implication as a tool for a real time external quality control and benchmarking.
\end{abstract}

\section{DGKL-P092}

\section{BANSAI: A novel approach for clinical chemistry analysis using laser light}

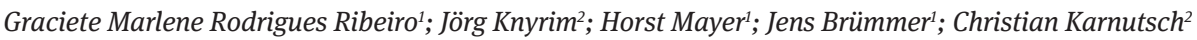

${ }^{1}$ Städtisches Klinikum Karlsruhe, Zentralinstitut für Laboratoriumsmedizin, Mikrobiologie und Transfusionsmedizin, Karlsruhe, Germany; ${ }^{2}$ Hochschule Karlsruhe, Research Group Integrated Optofluidics and Nanophotonics (IONAS), Karlsruhe, Germany

Background: Research within the group "Integrated Optofluidics and Nanophotonics (IONAS)" at Hochschule Karlsruhe is directed towards portable (Point-of-Care) biomedical analysis systems (e.g. blood analysis systems) employing organic semiconductor laser light sources (BANSAI). The aim is to develop novel concepts and technologies for POC clinical chemistry analysers at the quality of a central laboratory. In order to miniaturise the analysis system, we developed a novel lamination technology (Patent pending) that enables covering microfluidic channels with polymer thin-films. With this technology, on-chip laser sources for absorption spectroscopy, and thus potentially for clinical chemistry blood analysis, are feasible.

Aim of study: The aim of this study was to compare the photometric properties of the first implementation of a BANSAI system with a reference photometric system (Siemens Dimension Vista) to demonstrate BANSAI's clinical usefulness.

Materials and Methods: A novel photometric system (BANSAI) was evaluated and compared with a reference clinical chemistry analyser (Siemens DV) that uses a conventional lamp. The verification procedure for the performance of the BANSAI system consisted of imprecision, accuracy, lower limit of detection as well as linearity measurements of human albumin (HA) concentration in patient's material and pure lyophilized HA.

Results: The results of the within-day and between-days imprecision were constant: the average concentration of the low albumin control was $27.2 \mathrm{~g} / \mathrm{L}$, for the high albumin control $45.3 \mathrm{~g} / \mathrm{L}$ and the average concentration for the HA pool was $48.4 \mathrm{~g} / \mathrm{L}$. The average of the coefficient of variation $(\mathrm{CV})$ of the imprecision was $2.1 \%$ for low HA control, $1.9 \%$ for high HA control and 2.3\% for HA pool. Accuracy and associated recovery of controls are close to $100 \%$. Dilution series with pure HA was linear and a detection limit of $2.5 \mathrm{~g} / \mathrm{L}$ was determined. The BANSAI results were in very good agreement with those of an immunonephelometric assay.

Conclusion: Our results suggest that the BANSAI system is comparable to a photometric system of a conventional clinical chemistry analyser. A main finding was that the use of a second wavelength for compensation of possible matrix effects was not necessary for HA concentration measurements.

Furthermore, we were able to measure concentrations as low as $2.5 \mathrm{~g} / \mathrm{L}$, while the coefficient of variation stayed well below $10 \%$. This indicates that we may not have found the actual detection limit and that the system may be able to measure even lower concentrations. Succeeding in lowering the detection limit, the BANSAI system capabilities could be extended towards HA measurements in urine samples (reference range for adults is $0.02-0.2 \mathrm{~g} / \mathrm{L}$ ). 
Due to the use of organic lasers and the small number of components, a miniaturization of the system is possible, making it feasible for POC applications.

\title{
DGKL-P093
}

\section{Reference intervals with confidence intervals from small sample sizes}

\author{
Andreas Bietenbeck'; Wenxu Wei'; Frank Klawonn ${ }^{3}$ \\ ${ }^{1}$ Klinikum rechts der Isar der TU München, Institut für Klinische Chemie und Pathobiochemie, München, Germany; ${ }^{2}$ Ostfalia University, Wolfenbüttel, \\ Germany; ${ }^{3}$ Helmholtz Centre for Infection Research, Biostatistics, Braunschweig, Germany
}

\begin{abstract}
Background: Reference intervals are an important tool to assist in the interpretation of laboratory results. Traditionally, reference intervals are calculated as the central $95 \%$ of at least 120 measurements of a healthy reference population. However, this approach is expensive, timeconsuming and not practicable in cases such as for pediatric populations. Newer, indirect approaches estimate reference intervals from stored measurements. This is only possible if a sufficiently large number of measurements is already present. A third possibility is to adopt reference intervals provided by the manufacturer or from scientific literature. Not least because unique preanalytic factors might influence the measurement and thereby the reference interval, the adopted values need to be validated with patient measurements. For example, the CLSI Approved Guideline C28-A3c recommends to measure 20 samples and suggests that no more than two measurements may exceed the reference interval. Research Question: From the medical and statistical point of view, it is not sufficient to simply provide best guesses for reference limits for small sample sizes. The uncertainty of these estimates should also be quantified. Can we use methods from statistics to compute confidence intervals for reference limits derived from small sample sizes?

Material and Methods: Chakraborti and Li (2010) described a method to estimate confidence intervals for percentiles under the assumption that the sample follows a normal distribution. We have implemented the method to compute confidence intervals for the $2.5 \%$ and the $97.5 \%$ percentile that are required for reference limits.

Results: A tool with an easy to use graphical user interface has been implemented that first carries out tests for normality, outliers, and skewness in order to rule out confidence interval estimation based on inappropriate data. Afterwards it estimates reference limits and confidence intervals.

Discussion: The advantage of the presented approach is twofold. First, our algorithm can calculate reference intervals from a very small sample size. Therefore, efforts to establish new reference intervals or to validate existing specifications are considerably reduced. Second, our algorithm provides confidence intervals to assess uncertainty.

Theoretically, laboratory-specific reference intervals should provide better interpretation support because they are more adapted to the measurement at hand. However, reference intervals often have a variability unrelated to the measurement. With our easy-to-use tool, we hope to improve this situation.
\end{abstract}

\section{References:}

Jones, G.R. et. al., 2018. Indirect methods for reference interval determination-review and recommendations. Clinical Chemistry and Laboratory Medicine.

Jones, G.R., et. al., 2004. The case for common reference intervals. The Clinical Biochemist Reviews.

S. Chakraborti, J. Li (2010) Confidence Interval Estimation of a Normal Percentile. The American Statistician.

\section{DGKL-P094}

\section{Lab results are no strawberries - why we need to change what's red}

\author{
Christian Schäfer; Stefan Bollmann; Matthias Nauck; Astrid Petersmann \\ Universitätsmedizin Greifswald, Institut für Klinische Chemie und Laboratoriumsmedizin, Greifswald, Germany
}

Background: The results of laboratory testing are an important pillar in diagnostics and monitoring. A vast number of therapeutic decisions are mainly based on lab results and their interpretation. This interpretation is commonly performed by human medical staff.

Human cognitive capability for information uptake and information processing is rather limited compared to the vast amount of different laboratory parameters available. In different approaches and publications psychologists arrive at about four to nine items ("chunks") of information which can be recalled from working memory. Other works discuss not a fixed number approach but a concept of a limited memory resource in which relevance of information, precision of information and other stimuli compete.

The numerical result of an analysis needs accompanying information. The Rili-BAEK requires "reference intervals or other remarks for interpreting the examination results". In general results on lab reports are highlighted using red and/or bold print if their value is outside of the reference intervals (ref.int.). 
Ref.int. are usually obtained from a healthy cohort. Most clinical patients are unlikely to be considered healthy. This leads to the suggestion that examination results are likely to be out of the ref.int. at least for some parameters.

The question arises whether the amount of information returned for clinical interpretation may exceed human cognitive capabilities.

Material and Methods: We analyzed routine clinical chemistry orders of the university hospital Greifswald, Germany over the first quarter of 2018. The orders were either filed via digital order entry or paper form. Results which indicated missing material or technical difficulties were omitted. We counted the number of individual results printed (total) and number of results out of ref.int. (OORI) for each order and then analyzed distribution of total and results OORI.

Results: 47,020 orders matched our inclusion criteria. The orders had an average of 20.5 reported results (median 20). Only about $8 \%$ of the orders had all of their analytic results within the ref.int. An average of $32 \%$ of the results within an order were OORI (avg 6.6; median 5). More than 10 results were OORI for $22 \%$ of all orders $(n=10340)$.

Conclusion: Our investigation shows that a great number of lab orders have multiple OORIs. Lab results are in general reported cumulatively together with previous orders. Hence the number of items on one report is even greater than that of a single lab order. Therefore it is likely that a considerable number of lab reports exceed human memory resources.

Future rise to the number of reported results may be caused by OMICS technologies. Thereby even more information needing interpretation could be added.

This stresses the need for more comprehensive means of displaying lab results to physicians.

\section{DGKL-P095}

\section{Indirect Procedure for the Estimation of Reference Limits for Uric Acid}

Bernd Wolters ${ }^{1}$; Alexander Bertram²; Nicolas von Ahsen ${ }^{3}$

${ }^{1}$ St. Joseph-Stift Bremen, Institut für Laboratoriumsmedizin, Bremen, Germany; ${ }^{2}$ Amedes MVZ wagnerstibbe, Hannover, Hannover, Germany; ${ }^{3}$ Universitätsmedizin Göttingen, Institut für Klinische Chemie/UMG-Labor, Göttingen, Germany

\section{Introduction:}

Hyperuricemia is defined by uric acid levels above $6.8 \mathrm{mg} / \mathrm{dl}$ by the S2e AWMF guideline and is based on the solubility of sodium-urate in plasma water. There are studies showing increasing prevalence of hyperuricemia and gout. Besides this most laboratories use the reference limits reported by the manufacturer.

\section{Methods:}

Uric acid and creatinine from mainly inpatient (Lab 1, 01/2011-12/2017, N=101194, Roche c501), mainly outpatient (Lab 2, 05/2015-04/2018, $\mathrm{N}=339139$, Beckman-Coulter AU5800) and one university clinic (Lab 3, 01/2017-12/2017, N=32604, Abbott Architect) were retrieved from the information systems. For calculation patients first values with age 18-100 years were included. In a second step preselection was done and patients with elevated creatinine ( $>1.2 \mathrm{mg} / \mathrm{dl}$ male, $>0.9 \mathrm{mg} / \mathrm{dl}$ female) were excluded. The reference limits (RL) were calculated with the indirect method using the Reference Limit Estimator (version 49).

\section{Results:}

\begin{tabular}{|c|c|c|c|c|}
\hline $\mathrm{mg} / \mathrm{dl}$ & & $\mathbf{N}$ & female & male \\
\hline \multirow[t]{3}{*}{ Lab 1 (serum) } & all patients & 59769 & $2.5-8.8$ & $3.3-8.8$ \\
\hline & & creatinine $<$ RL 41565 & $2.4-7.1$ & $3.2-8.5$ \\
\hline & & Roche Diagnostics c501 & $2.4-5.7$ & $3.4-7.0$ \\
\hline \multirow[t]{3}{*}{ Lab 2 (serum) } & all patients & 112302 & $2.8-8.0$ & 3.9-9.1 \\
\hline & & creatinine $<$ RL 104578 & $2.8-7.6$ & $3.9-8.9$ \\
\hline & & Beckman-Coulter AU5800 & $2.3-6.6$ & 4.4-7.6 \\
\hline \multirow[t]{3}{*}{ Lab 3 (plasma) } & all patients & 13975 & $2.7-9.2$ & 3.7-9.9 \\
\hline & & creatinine $<$ RL 10487 & $2.7-8.1$ & $3.5-9.2$ \\
\hline & & Abbott Architect & $2.6-6.0$ & $3.5-7.2$ \\
\hline
\end{tabular}

The exclusion of intensive care units (Lab 1) did not influence the results. The estimated RL were higher than the RL reported by the manufacturer. Additionally we found continuously increasing upper RL for (postmenopausal) women.

Discussion: We found higher upper RL than recommended by the manufacturer with all systems. The preselection of patients with elevated creatinine was done to exclude patients with renal insufficiency. The exclusion of oncology patients may be reasonable but was not possible and only some tumors may lead to increased uric acid.

Most likely the actual limits need to be elevated due to change in food pattern. However, we recommend the initiation of a prospective study for the estimation of RL using a direct method. Additionally we found increasing upper RL for postmenopausal women which supports the concept of continuous reference limits. Above all the therapeutic aim of uric acid lowering therapy ( $<6 \mathrm{mg} / \mathrm{dl}$ ) is defined by outcome studies. 


\title{
DGKL-P096
}

\section{Blood group based detection of wrong-blood-in-tube in clinical chemistry blood samples}

\author{
Thorsten Weiland; Severin Hahn \\ MEDILYS Laborgesellschaft mbH, Immunhämatologie, Hamburg, Germany; MEDILYS Laborgesellschaft mbH, Immunhämatologie, Hamburg
}

Background: Specimen or patient misidentification at the time of blood drawing ("wrong blood in tube” (WBIT)) represents a common problem in clinical chemistry laboratories with the potential of serious hazards for patients. Though the rate of WBIT is well known for densely regulated pretransfusion samples, we are not aware of objectively generated data for routine clinical chemistry samples. The WBIT rates in clinical chemistry samples published so far are calculated from "soft" detection mechanisms with unknown sensitivity and specificity. Our study applied ABO and RhD-typing to standard EDTA samples submitted for complete blood counts (CBC) as an objective method of error detection and compared this to traditional delta check performance.

Methods: Standard ABO and RhD-typing by automated gel centrifugation test was applied during seven consecutive days to all EDTA samples sent in for CBC. No additional blood samples were drawn for these ABD-typings. ABD results of each patient were compared with either preexisting blood type data or with previous ABD results within this study. Results remained blinded until after the end of the study period. We also took our standard detection methods into account (delta check, hospital staff reporting).

Results: 2475 specimens from 1422 patients were typed for ABD blood groups. 1688 specimen from 793 patients had preexisting ABD data. Of these, 3 samples were found to be WBITs due to ABD-discrepancies. All 3 WBITs had also been detected by routine delta check procedures. 2 additional WBITs in this population were detected after result reporting by hospital staff. These two cases had been detected neither by ABD typing (both the correct and the wrong patient typed identical for ABD) nor by delta checks. The overall WBIT rate therefore was 3.0/1000 samples. Adapted for silent errors (factor 1.37 for ABD typing) the WBIT rate increased to 3.6/1000 samples for the combined rate and to 2.4/1000 samples detected by ABD typing only.

Summary: Our study finds a combined WBIT rate of 3.0/1000 samples (3.6/1000 samples adapted for silent errors) in routine clinical chemistry samples, which is 5 to 8 times higher than commonly reported numbers in pretransfusion samples. This might result from a supposed lower risk for patients from misidentified clinical chemistry samples and thus less strict safety measures when drawing those blood samples. Benchmark data from several regional hospitals (using the traditional detection methods) does not suggest an elevated WBIT rate in the specific study Hospital only.

The fact that ABD-typing revealed no additional WBIT compared to routine procedures (delta check and hospital reporting) is unexpected and raises doubt about the representability of our study period. We encourage further studies in different institutions and populations to achieve objective WBIT rates for clinical laboratories thus helping to reduce this potentially hazardous phenomenon.

\section{DGKL-P097}

\section{Determination of selected steroid hormones from saliva by mass spectrometry}

Jonas Schmidt ${ }^{1}$; Frithjof Blessing ${ }^{2}$; Folker Wenzel ${ }^{1}$

${ }^{1}$ Hochschule Furtwangen, Medical and Life Sciences, Villingen Schwenningen; ${ }^{2}$ Laborärzte Singen, Leiter, Singen, Germany

Intoduction: Steroid hormone analysis from saliva has become more and more popular in the recent years and therefore the demand for such analyses has increased in the routine laboratory. In general the analysis of salivary steroid hormones is a challenging task due to the low physiological concentration ranges and the poor ionizability of these compounds. Therefore, it is crucial to use sensitive and selective analytical methods to get reliable results.

Material and Methods: The aim of the current study is to investigate the applicability of liquid chroma-tography-mass spectrometry (LC/MS) for the quantification of cortisol, testosterone, progesterone and estradiol from saliva For this purpose initially a suitable mass spectrometric method was developed by optimizing the parameters of the mass spectrometer, the chromatographic conditions as well as the sample preparation. For sample preparation solid phase extraction (SPE) and derivatization with hydrox-ylamine was used to increase sensitivity. After method development a validation was performed by in-vestigating limit of detection (LOD), limit of quantification (LOQ), linearity as well as recovery and precision. Finally, a method comparison was performed only for cortisol by measuring 17 routine saliva samples using LC/MS and a commercial immunoassay kit from IBL International.

Results: LC/MS in combination with hydroxylamine derivatization is capable of detecting cortisol, testos-terone and progesterone from saliva in principle. The detection of estradiol is not possible by using this derivatization technique. However, the validation measurements showed that the Waters Xevo TQ MS triple quadrupole mass spectrometer which was used for analysis is not sensitive enough to detect low physiological concentrations of testosterone and progesterone in saliva although derivatization and SPE were used to increase sensitivity.

Conclusion: The method comparison for cortisol demonstrated that the immunoassay seems to overes-timate salivary cortisol concentration in comparison to mass spectrometry. This phenomenon mainly occurs due to unspecific reactions of the antibodies used in an immunoassay for detection of the target analyte. 


\title{
DGKL-P098
}

\section{Combining extraction by precipitation and fluorescent nanoparticle tracking analysis for rapid and reproducible quantification of small extracellular vesicles in human plasma samples}

\author{
Ritika Sharma; Michael Kiehntopf; Boris Betz \\ Universitätsklinikum Jena, Institut für Klinische Chemie und Laboratoriumsmedizin, Jena, Germany
}

Introduction: Extracellular vesicles (EVs) are membranous particles in the submicron range $(<1000 \mathrm{~nm})$ that are constitutively released by cells and are involved in many physiological functions like cell-cell communication. The quantification of small EVs $(<200 \mathrm{~nm})$ in plasma remains a challenging task due to the limited resolution of quantification techniques and due to the high background noise in plasma.

Methods: The Total Exosome Isolation Reagent (ThermoFisher) was used to quickly isolate frozen plasma samples without any known disease. Since the isolates from precipitation kits are known to be contaminated with non-EV small particles the fluorescent Nanoparticle Tracking Analysis (fNTA) was applied for specific quantification using the ZetaView Analyzer (Particle Metrix) and the Qtracker labelling kit (ThermoFisher). The detergent Triton $(0,1 \%)$ was used to destroy the EV membrane structure. The intra-assay and inter-assay coefficients of variation (CV) were calculated according to CLSI guidelines.

Results: The total fluorescence (size range $0 \mathrm{~nm}-400 \mathrm{~nm}$ ) did not differ between blanks and plasma samples (-/ + Triton 0,1\%) and represents the total bound and unbound concentration of Qtracker fluorescent particles (Qdots). Therefore, by correcting for the total fluorescence the potential interference by bleaching effects might be minimized.

In comparison to the blank (water) all plasma samples had a markedly higher concentration for fluorescent particles in the size range between $100 \mathrm{~nm}$ and $200 \mathrm{~nm}$. This effect could be reversed by addition of Triton $0,1 \%$ to the sample.

The CV results were in an acceptable range below 10\% (intra-assay) or 20\% (inter-assay). CVs were also constant in a dilution series.

Conclusion: By combining the precipitation method and fNTA a reliable and rapid method for the quantification of small EVs in human plasma samples is provided. To our knowledge, the intra- and inter-assay CV for fNTA in human plasma samples is reported for the first time for the ZetaView Analyzer.

\section{DGKL-P099}

\section{Introducing the method of minimal difference into cerebrospinal fluid protein analysis}

Marie Süße ${ }^{1}$; Malte Hannich ${ }^{2}$; Astrid Petersmann ${ }^{2}$

${ }^{1}$ Universitätsmedizin Greifswald, Klinik und Poliklinik für Neurologie, Greifswald, Germany; ${ }^{2}$ Universitätsmedizin Greifswald, Institut für klinische Chemie und Laboratoriumsmedizin, Greifswald

Objective: The basis of CSF (cerebrospinal fluid) protein analysis is the use of CSF/serum quotients (albumin quotient (QAlb), immunoglobulin G quotient (QIgG), immunoglobulin A quotient (QIgA), immunoglobulin M quotient (QIgM)) and their presentation in the so called "Reibergramm".

In 2004 Reiber et al. published a consensus protocol on laboratory techniques to improve the quality of analysis and interpretation of CSF measurements. The authors argued that CSF and serum proteins need to be measured on the same platform with the same calibration to insure method-independent high sensitivity and specificity. It was recommended that the CSF/Serum quotient should be taken into account as part of the final evaluation.

In July 2016 Siemens announced, that they no longer provide the nephelometric serum immunoglobulin M assay for the BN Prospec, which is widely used in CSF laboratories, forcing them to change the complete serum analysis to another platform.

A rarely used though handy tool to illustrate the variation of quality control measurements is the MD (Minimal Difference [ $\left.k^{\star} \mathrm{SD}^{\star} \sqrt{ } 2\right]$ ). It bears the advantage of giving specific concentrations as absolute values, thus making interpretation more intuitively.

We evaluated retrospectively the quality control measurement of CSF and serum on two different platforms by applying the MD to the albumin and immunoglobulin quotients.

Methods: Internal laboratory quality control results of CSF and serum were used, comprising two different levels measured daily, to calculate different quotients according to current protocol by Reiber et al. We used the MD of the quotients to verify if the combined results of the two used platforms were within the accepted ranges given by the German Rili-BÄK. The target values given by the manufacturers were used to determine the given reference range.

Albumin (nephelometric), IgG, IgA and IgM (particle enhanced nephelometry) were measured on the BN Prospec (Siemens Healthcare Diagnostics, Eschborn, Germany). Serum albumin, IgG, IgA and IgM (photometric) were measured on the Siemens Dimension Vista (Siemens Healthcare Diagnostics). Measurements and calibrations were conducted according to the manufacturer's protocol.

Results: Albumin quotients showed an MD ranging from 0.32 to 1.43 (CV (coefficient of variation): 3.41-3.97). Immunoglobulin quotients MD ranged for IgM from 0.74-2.93 (CV: 9.08-9. 24), for IgA from 0.42-3.42 (CV: 8.58-9.01), for IgG from 0.20-0.95 (CV: 3.57-4.36). Based on the established target values for each quotient all MD’s were within the respective reference range. 
Significance: The results of our work show, that the use of two different measuring platforms allows working within the specifications given by the German Rili-BÄK. The MD could be a handy tool to illustrate quality control results in the CSF laboratory. An integration into the "Reibergramm" could facilitate its use in laboratory workflow.

\section{DGKL-P100}

\section{Analysis of the Factors Affecting the in vitro Hemolysis and Implementation for Identification of Potential Pre- Analytical Errors}

Feodora Trifonova; Sebastian Wegerer; Michael Neumaier; Karin Janetzko Universitätsmedizin Mannheim, Institut für klinische Chemie, Mannheim, Germany

As an indicator for sample integrity the hemolysis index (HI) plays an important role in the quality control and management in a certified clinical laboratory. We analyzed and reported our data of the HI of more than 800,000 samples assessed in our laboratory over a forty-onemonth-period. Our study confirms the dependency of the in vitro hemolysis on various factors such as age of the patients, specimen transportation type etc. A striking difference and a significantly higher HI were registered in the emergency room as an indicator of how the rushed processing influences the quality of the blood samples. In our hands the $\mathrm{HI}$ is a tool for identifying potentially vulnerable and problematic situations on the way from patient to the lab.

\section{Posterwalk Analytische Qualität und Labormanagement II}

\section{DGKL-P101}

Integrated, automated biobank sampling

Abass Eidizadeh ${ }^{1}$; Moritz Schnelle ${ }^{1}$; Sara Y. Nussbeck ${ }^{2}$; Daniela Popp ${ }^{2}$; Jutta Engelmayer ${ }^{1}$; A. R. Asif ${ }^{1}$; Lutz Binder ${ }^{1}$

${ }^{1}$ Universitätsmedizin Göttingen, Institut für Klinische Chemie, Göttingen, Germany;

${ }^{2}$ Universitätsmedizin Göttingen, UMG Biobank, Göttingen, Germany

Background: During the past few years, there is a steeply growing interest in novel biomarkers on the part of universities, research institutes and industrial companies. Scientific journals publishing articles on biomarkers still demand very little information from the authors on the quality of the investigated samples. However, marker instability under uncontrolled circumstances of preanalytical sample processing may lead to in vitro test results being considerably discrepant from the in vivo situation. Next to archiving conditions, the influence of preanalytical sample handling is of crucial importance.

Aim: The aim of the present project is the implementation of a rapid, standardized and traceable pre-freezing liquid handling process for highest biobank sample quality.

Results: The overall biobanking process starts on the ward and resembles the existing blood sampling process for patient care. Different biobanking profiles (concerning sample materials) are electronically offered according to the sampling strategies of the different studies performed in the hospital. Biobank primary samples are prepared by the order entry system; barcode labels with order numbers are printed and pasted on the sample tubes. Patients carry bracelets with their barcoded ID. By scanning the barcode immediately prior to blood collection, the patient's correct identity is guaranteed and the actual sampling time is registered.

After sample transport to the central laboratory, arrival time is documented. Sample tubes are fed into the bulk loader, directly linked to a laboratory automation system (LAS) with conveyor belt, centrifuges, analyzers, cooling archive, and the "liquid handling robot" (LHR). Independent from staff intervention, samples are automatically put into centrifuges, decapped, transported to the LHR and made available for aliquotation. In the LHR, 2D barcoded, smaller secondary biobanking tubes are identified, decapped, aliquoted, recapped, and provided for freezing in a 1D barcode-labeled rack. Rack provision time is documented as well as the time when the rack is frozen at a temperature below $150{ }^{\circ} \mathrm{C}$. The complete sample related process data are available in the LIS and will later be transferred via an interface into the biomaterial information management system.

Level detection by the electrically conducting pipetting tips, however, may result in cell aspiration from grossly underfilled primary sample tubes. Furthermore, aliquoting of buffy coat is not yet available in an automated manner.

Conclusion: The introduction of a standardized, automated and integrated system for biobank sample collection allows provision of high quality samples from bed to freezer within approximately 60 minutes. All relevant sub-processes are temporally documented. For the described process, study nurses are no longer required. Due to automation, human sources of error are largely excluded. 


\title{
DGKL-P102
}

\section{Method Comparison and Work Flow Optimization of Urinary Stone Analysis}

Katrin Höland; Romy Schär; Jeannine Joneli; Monika Reusser; Yoland Aebi; Ruedi Bühlmann; Jean-Marc Nuoffer; Martin Fiedler; Nazanin Sédille-Mostafaie

Inselspital, Bern University Hospital, and University of Bern, Center of Laboratory Medicine (ZLM)/University Institute of Clinical Chemistry, Bern, Switzerland

Urolithiasis, the pathological formation of biomineralized calculi, is a widespread disease with increasing incidences in industrialized countries. It additionally possesses a high recurrence risk with up to $50-80 \%$. Since urinary stone formation is only the symptom of an underlying disease, correct and efficient urinary stone analysis, i.e. the determination of the composition of the urinary calculi, is of importance. It is the basis for the definition of the disease etiology, for additional diagnostic decisions, and for appropriate surgical, medical and nutritional treatments, which ultimately allow a decrease in recurrence risk.

Data base analysis of all urinary stone samples analyzed at the Center of Laboratory Medicine at the Bern University Hospital between 2008 and 2018 was performed $(n=6774)$. This allowed the determination of the most common urinary stone types analyzed over the past ten years. Instrument and method comparison were performed using the Spectrum One Fourier-transform infrared (FTIR) spectrometer with potassium bromide (KBr) pellets and the Spectrum Two FTIR spectrometer with $\mathrm{KBr}$ pellets as well as with the universal attenuated total reflection (UATR) accessory (all PerkinElmer). The samples measured with all three techniques included: all routine urinary stones during a period of three weeks $(n=62)$, external quality control material $(n=32)$ and urinary calculi from a collection present at the Center of Laboratory Medicine $(n=6)$. Calculi composition was determined by comparison of the measured spectrum to spectra libraries (obtained by the manufacturer and in-house). Further, intra- and inter-assay comparisons $(\mathrm{n}=10)$ were performed and $\mathrm{KBr}$ pellet stability was tested.

The most common urinary stone types analyzed at the Center of Laboratory Medicine during a time period of ten years include weddellitwhewellit combinations, carbonate apatite-weddellit-whewellit combinations and pure whewellit concrements. Preliminary data of the instrument and method comparison show good agreement between both $\mathrm{KBr}$ pellet techniques on Spectrum One FTIR spectrometer versus Spectrum Two FTIR spectrometer, using the same spectra libraries for composition determination. In the majority of samples, UATR and KBr pellet techniques show good agreement in detecting the same main component but vary in the percent composition of the urinary calculi. Apart from analytical results, the workflow can be optimized by using the UATR application, since preparation of the KBr pellet is not necessary. The turnaround time can thereby be reduced by approximately $15 \mathrm{~min}$ (45\%) for each analysis (complete process from arrival of the urinary concrement at the laboratory bench until release of the results in the laboratory information system).

\section{DGKL-P103}

\section{Assay-dependent differences in PCT measurements}

\author{
Abass Eidizadeh; Mechthild Wiederhold; Nicolas von Ahsen; Lutz Binder; Moritz Schnelle \\ Universitätsmedizin Göttingen, Institut für Klinische Chemie, Göttingen, Germany
}

Background: Procalcitonin (PCT) is a widely used biomarker for systemic inflammation und bacterial infection. In clinical practice, assessment of PCT is a diagnostic tool (i) to estimate the severity of bacterial/fungal infections, (ii) to distinguish bacterial/fungal from viral infections and non-infectious inflammatory conditions, and (iii) to monitor efficacy of antibiotic treatment. There are several companies offering PCT immunoassays for patient care (i.e. Abbott, Roche, DiaSorin), but a reference method and a reference material are missing. Thus, we evaluated three automated PCT assays with respect to their comparability.

Methods: PCT values in plasma from 64 randomly selected patients were measured using Liaison XL (DiaSorin), Cobas e411 (Roche) and Architect i2000SR (Abbott). Comparability was assessed performing Passing-Bablok regression analysis; imprecision was calculated according to the CLSI EP15 A2 protocol.

Results: Comparison of PCT values between all three analyzers showed good correlations with $\mathrm{r} \geq 0.995$. However, regression analysis revealed relevant discrepancies between Liaison and the other two analyzers with slopes of 1.715 (vs. Architect) and 1.789 (vs. Cobas). On average, PCT values from Liaison were 40\% higher compared to Architect and Cobas. In contrast, Architect and Cobas displayed comparable values (slope: 0.957). Calibrator cross-checks confirmed these findings. All three analyzers showed acceptable imprecisions with CVs ranging from 2.3 to $6.1 \%$.

Conclusion: Differences in measured PCT concentrations may favour false medical decisions in patient treatment. Our data revealed constantly higher PCT values in Liaison compared to Architect and Cobas indicating a systematic deviation. According to our data, this may be a consequence of incorrect calibrator set points. The presented findings highlight the need for a standardized reference material to guarantee comparable measurements in different analyzers. 


\title{
DGKL-P104
}

\section{Evaluation of VACUETTE® CAT Serum Fast Separator Blood Collection Tube for Routine Chemistry Assays}

\author{
Sirid Griebenow \\ Greiner Bio-One GmbH, Produkt Management, Kremsmünster, Austria
}

Background: Clotting time is an important factor in the work flow of blood collection tubes for routine chemistry testing from serum. To optimize laboratory workflow, reduced turnaround times are expected to provide precise test results. The key target of VACUETTE® CAT Serum Fast tubes consists in the faster processing time from blood collection to result availability. The faster clotting time in the VACUETTE® CAT Serum Fast tube provides a clotted sample by the time the sample reaches the laboratory and allows for immediate testing. Tubes containing a gel separator offer the option of replicate measurements up to $48 \mathrm{~h}$.

Method: This study was conducted in order to demonstrate the performance of VACUETTE® CAT Serum Fast tubes for routine chemistry analysis up to $48 \mathrm{~h}$ in comparison to VACUETTE® Serum Separator tubes. Venous blood was drawn from 50 healthy donors into two tubes. Tubes without thrombin were centrifuged after $30 \mathrm{~min}$ clotting time, and the tubes with thrombin were centrifuged after 5 min clotting time. All samples were centrifuged for $10 \mathrm{~min}$ at 1800g. Initial values of routine chemistry assays were determined on an AU680 and DxI800 from Beckman Coulter (Beckman Coulter, precision within-run $<3 \%$, total $<3 \%$ ). All samples were stored in an upright position at 4- ${ }^{\circ} \mathrm{C}$ for replicate testing after $24 \mathrm{~h}$ and $48 \mathrm{~h}$. Comparison analysis was performed at all time points. Clinical evaluation was based on CLIA (Allowable Total Error Table by Data Innovations).

Results: Equivalency for VACUETTE® CAT Serum Fast tubes to VACUETTE® Serum Separator tubes was shown for routine chemistry assays on Beckman Coulter for healthy donors. Provided a completely clotted sample and clear serum specimens, no significant deviations were found for initial values as well as at $48 \mathrm{~h}$ for 37 biochemical assays tested in both tubes according to CLIA tolerances. In agreement to literature, slight systematical deviations in the thrombin tubes were found for some assays such as sodium, potassium, chloride, and glucose due to the faster coagulation process. Stability over $48 \mathrm{~h}$ was shown for all assays except troponin I (6h).

Conclusion: The thrombin tube gave comparable test results to current serum separator tubes for most common biochemical assays in clinical laboratories. The blood collection tube containing thrombin provides rapid turnaround times in the laboratory by shortening the clotting time, providing accurate testing results and being suitable for emergency testing, however, those tubes are not recommended for patients on heparin therapy, thrombin inhibitor therapy or with deficiency in the clotting factors.

\section{DGKL-P105}

\section{Evaluation of a new pneumatic tube system carrier}

Cora Milena Märtens; Juliane Suchsland; Stefan Bollmann; Anke Hannemann; Stephanie Zylla; Matthias Nauck; Astrid Petersmann ${ }^{1}$ Universitätsmedizin Greifswald, Institut für Klinische Chemie und Laboratoriumsmedizin, Greifswald, Germany

\section{Objectives:}

A new carrier by Aerocom ${ }^{\circledR}$ for pneumatic tube systems (PTS) is able to transport 9 blood tubes while mechanically fixing the samples within the carrier during transport. It does not require any special packing and is able to release samples by an automatic unloading station, e.g. directly into a bulk loader. In this study we examine the influences of the transport using the new carrier on blood sample quality and compare it to conventional carriers and courier transport.

\section{Methods:}

Triplicate blood samples sets (1 EDTA, 1 Lithium Heparin, 1 Citrate) of 30 volunteers were split among the different transportation methods 1. courier, 2. conventional PTS carrier and 3. new PTS carrier Aerocom ${ }^{\circledR}$. During transport a mini data logger (MSR145®) was used to continuously measure the accelerations in three axes in the new carrier. After transport 53 parameters from clinical chemistry, hematology and coagulation were measured and compared. The corresponding accelerations were evaluated according to Streichert et al [1].

\section{Results:}

For all analytes we compared absolute and relative differences of medians. For LDH and potassium relative differences of medians were $2.45 \%$ and $2.63 \%$ respectively compared to courier and $0.34 \%$ and $0 \%$ compared to conventional carriers. There was no difference between medians for ASAT in comparison to courier and 5\% compared to conventional carriers. The analytical comparisons did not show relevant differences between the new carrier and courier or conventional carriers.

Conclusion:

The new carrier by Aerocom ${ }^{\circledR}$ is suitable for transporting diagnostic blood samples with the orders for the investigated parameters. The overall workflow is improved by decreasing hands on time on the ward and in the laboratory and by minimizing the risk of incorrectly packed carriers.

\section{References:}

1. Streichert T, Otto B, Schnabel C, Nordholt G, Haddad M, Maric M, et al. Determination of hemolysis thresholds by the use of data loggers in pneumatic tube systems. Clin Chem 2011;57:1390-7. 


\title{
DGKL-P106
}

\section{Comparative measurements of Procalcitonin with two different assays on various analytical platforms}

\author{
Konstantin Gröber ${ }^{1}$; Martina Zogbaum ${ }^{1}$; Anke Pigulla ${ }^{2}$; Thomas Demant ${ }^{1}$ \\ ${ }^{1}$ Städtisches Klinikum Dresden, Institut für Klinische Chemie und Labormedizin, Dresden, Germany; ${ }^{2}$ Diakonissenkrankenhaus Dresden, \\ Zentrallabor, Dresden, Germany
}

Background: Procalcitonin (PCT) is well established in the diagnosis and therapeutic monitoring of bacterial sepsis. It can be determined by at least two different immunological assays but in the absence of a reference method the true PCT values in patient samples remain elusive. By comparison of two immunological PCT assays applied on four different analyzers we sought to find out the degree of interassay variation and its significance for clinical interpretation of results.

Methods: The PCT chemiluminescence immunoassay from Brahms/Thermofisher was applied on the Liaison immunoanalyzer from DiaSorin (method CL-1) and the Cobas e601-module from Roche (method CL-2). Similarly, the PCT turbidimetric assay from Diazyme was applied on the Cobas c501-module from Roche (method IT-1) and the UniCel DxC 600 immuno-analyzer from Beckman-Coulter (method IT-2). Samples from 338 patients from surgical intensive care units with a clinical diagnosis of suspected or established bacterial sepsis were analyzed for PCT by at least two different methods. Results were compared by regression analysis using the Acomed statistics Passing-Bablok regression software package. In a small subset of patients results were compared with a rapid quantitative teststrip for PCT from Suresign Finecare.

Results: Pair-wise comparison of the chemiluminescent assays (CL-1 vs CL-2) and the turbidimetric assays (IT-1 vs IT-2) demonstrated significant differences between methods with values for slope or intercept outside the range of $95 \%$ confidence intervals. Likewise, both immuno-turbidimetric assays were significantly different from the chemiluminescent assay either applied on the DiaSorin analyzer (CL-1) or on the Roche analyzer (CL-2). Best agreement between assays was reached when a subset of 87 patients was analyzed by method CL-2 and IT-1. Agreement of the PCT teststrip results was better with the chemiluminescent than with the turbidimetric assays but in any case lacked significance.

Conclusions: Regardless of which PCT assays and analytical platforms were compared, none of the methods for PCT measurements were sufficiently similar to be used interchangeably in clinical practice. In the absence of a reference method independent clinical evaluations with determination of clinical decision thresholds are paramount.

\section{DGKL-P107}

\section{Comparison of the dertermination of glucose in NaF- and GlucoExact sample material directly after blood collection}

\author{
Klaus-Martin Otte \\ Medilys Laborgeselschaft, Labor, Hamburg, Germany
}

Introduction: The effective inhibition of glycolysis to stabilize glucose requires an acidic solution of citrate in addition to NaF. Better stability over 24 hours ore more of a specimen with an additional citrate buffer compared to only NaF has been described repeatedly in the literature. The GlucoExact tube ( $2.7 \mathrm{ml}$, Sarstedt.) contains a liquid preparation of buffered citrate solution.

In the hospital, the transport times are usually much shorter. Therefore, the aim of the present investigation was to verify whether or not the values between the $\mathrm{NaF}$ ( $2.7 \mathrm{ml}$, Sarstedt) samples and the GlucoExact samples tubes are different under the conditions of process times $<30$ min after blood sampling and measurement.

Material and methods: The sample of 111 patients who visit our laboratory ambulance for blood glucose determination were simultaneously collected a NaF - and a GlucoExact tube. Both tubes were immediately centrifuge 10 min with RCF of 3500 in a refrigerated centrifuge. The quantitative determination of glucose concentration is carried out immediately afterwards on the architect C8000 (Abbott) with the hexokinase-/G-6-PDH-method. All measurements proceed at a time $<30 \mathrm{~min}$ after blood collection. The readings of the GlucoExact tube were multiplied according to the manufacturer by a factor of 1.16 .

The correlation analysis was performed with abacus 2.0, after Passing- Bablok. The statistical analysis was done with the Wilcoxon test for nonparametric tests with the SPSS program.

Results:

The comparison shows a correlation (Pearson ) of 0,998, the slope of 1.044 (regression equation: $\mathrm{y}=1,044 \mathrm{x}+1.044$ ).

The average value for the $\mathrm{NaF}$ sample material is $120.5 \mathrm{mg} / \mathrm{dl}$, for Glucoexact $127,0 \mathrm{mg} / \mathrm{dl}$

The difference of the value located between the two samples is significant $(\mathrm{p}<0.001)$.

Discussion: The values of glucose determination in the GlucoExact sample material is about $5 \%$ higher than the values in the NaF samples. This difference appeared after a very short period of time by $<30$ min between blood collection and measurement of the samples. Whether this difference is actually caused by an ineffective inhibition of glycolysis in the NaF tube or the acidic Citrat solution in the Glucoexact preparation itself cause the elevated values, can be judged here not definitively. The difference is however rather low at roughly $5 \%$, however, may be relevant for example to the decision boundaries for the glucose tolerance test. 
This investigation shows, that also in hospitals with short time between blood collection and processing a shift of the glucose values is to consider when using the Glucoexact sample material.

For the statistical analysis using SPSS, I thank Mr PD Dr. med Bruno Neuner, Charité, Berlin.

\section{DGKL-P108}

\section{Considerable discrepancies between rapid troponin testing and conventional laboratory analysis: can the use of these quick tests be justified?}

Christoph Frohn

Labor Dr Kramer und Kollegen MVZ GmbH, Immunhämatologie / Durchflusszytometrie, Geesthacht, Germany

Aims:

Review of the analytical quality of rapid troponin testing using the lateral-flow technique as compared with conventional laboratory analysis Methods:

Testing took place in a 186-bed district hospital in northern Germany with a cardiac-catheterization laboratory. Prompted by equipment failure over a four-day period in March 2015, samples were analysed using rapid testing as per the back-up system we had in place at the time. All rapid tests were used according to the manufacturers' specifications and evaluated by healthcare professionals.

As soon as the equipment (Tosoh AIA 360) had been repaired, it was used to retest the samples stored at $4{ }^{\circ} \mathrm{C}$. To eliminate any false positives generated by the Tosoh system, all samples were retested at the LADR Central Laboratory in Geesthacht (Roche/Cobas E 411, troponin I and troponin T). Additionally, in a second run of testing, the samples were tested by a different laboratory assistant using the same rapid test; there were no divergent results.

In the following weeks, samples that had tested positive using the Tosoh analyser were rechecked using various rapid tests to ascertain whether the unexpected frequency of test failures is reproducible.

The following rapid tests were employed:

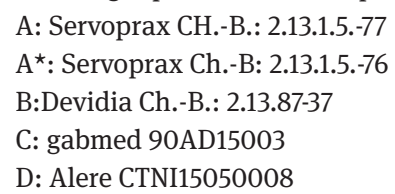

Results:

- Three out of four samples yielded a false negative result under the conditions of equipment failure and taking into account the specified sensitivity of the rapid tests.

- In systematic retesting, too, only four out of 23 positive samples were correctly identified using the originally used batch.

- Other tests proved, at best, only marginally superior.

- In our series, the detection limit was an estimated $6 \mathrm{ng} / \mathrm{ml}$, equivalent to 12 times that specified, and 100 times the limit for diagnosing myocardial infarction that is recommended in the guidelines.

- $\quad$ The results from the two fixed units used (Tosoh and Roche /Cobas) were in very good agreement.

\section{Summary:}

- Troponin assays are of paramount importance in diagnosis of myocardial infarction.

- Lateral-flow rapid tests are an attractive option for users as the result is available immediately without any special equipment requirements.

- $\quad$ The incidence of false-negative outcomes was unacceptably high for all manufacturers and batches investigated.

- Incorrect analysis led to patients being discharged prematurely.

- We cannot account for the discrepancies from the manufacturers' specifications.

- $\quad$ Our overall conclusion is that all of the troponin rapid tests are unsuitable as a basis for clinical decision-making.

\section{References:}

1) 2015 ESC Guidelines for the Management of Acute Coronary Syndromes in Patients Presenting without Persistent ST-segment Elevation European Heart Journal, 2015 - doi: 10.1093/eurheartj/ehv320

2) M. Oehler; Der Allgemeinarzt, 2014; 36 (20) pp. 78-80

Verdacht: Herzinfarkt; Fallstricke in der Frühphase 


\title{
DGKL-P109
}

\section{Influence of the fast gel separation barrier in the new S-Monovette® LH Gel+ on selected analytes}

\author{
Frithjof Scheer \\ SARSTEDT AG \& Co. KG, Entwicklung, Nümbrecht, Germany
}

Background: Gel separation barriers are widely used in blood collection tubes to separate plasma or serum from cells after centrifugation and thus allow prolonged storage of these samples. These gels are commonly polyacrylate based mixtures and usually have no influence on analytes. To confirm this, the S-Monovette ${ }^{\circledR}$ LH Gel + was compared to a S-Monovette ${ }^{\circledR}$ LH Gel and a S-Monovette ${ }^{\circledR}$ LH without gel. In total 57 analytes were measured from these tubes at three different time points spanning over seven days.

Material and methods: Blood was collected from 30 healthy adult donors into three different tubes, according to manufacturer instructions: two lithium heparin tubes with different polyacrylate gels (Sarstedt S-Monovette ${ }^{\circ}$ REF 04.1940 and 04.1954) and a lithium heparin tube without any barrier (Sarstedt S-Monovette ${ }^{\circledR}$ REF 04.1936). Within 30 minutes the tubes were centrifuged at $3000 \mathrm{~g}$ for $10 \mathrm{minutes}$. To avoid influences from different conditions, all tubes were centrifuged at the same speed and duration. A physical barrier (Sarstedt Seraplas ${ }^{\circledR}$ Filter REF 53.419) was placed immediately into the tube without gel to separate plasma and cells. For 11 analytes, blood was spiked, distributed to the tubes and processed as described above. The analytes were measured within 7 hours after centrifugation, one day later and after seven days on Roche cobas c 702 or e 602 analyzers. During this time the tubes were stored at 2-8 ${ }^{\circ} \mathrm{C}$. Statistical analysis employed Passing-Bablok regression according to CLIA guidelines.

Results: There were no clinical significant differences between the three S-Monovettes. The fast gel of the S-Monovette ${ }^{\circledR}$ LH Gel + has no influence on any of the 57 analytes. All analytes can be stored for seven days, except for glucose and potassium, which can be stored for one day.

Conclusion: The fast gel of the S-Monovette ${ }^{\circ} \mathrm{LH}$ Gel +, which allows very short centrifugation conditions down to 4 Minutes, has no influence on the 57 analytes of this study. Thus the S-Monovette ${ }^{\circ}$ LH Gel + is clinically equivalent to the S-Monovette ${ }^{\circ}$ LH Gel and the S-Monovette ${ }^{\circledR}$ LH without gel.

\section{DGKL-P110}

\section{Rapid centrifugation doesn`t do the trick- BD Barricor tubes are no alternative for Greiner glucose tubes}

\author{
Theresa Winter; Anke Hannemann; Astrid Petersmann; Matthias Nauck \\ Universitätsmedizin Greifswald, Institut für Klinische Chemie und Laboratoriumsmedizin, Greifswald, Germany
}

Introduction: Greiner Vacuette ${ }^{\circ}$ FC-Mix glucose tubes stabilize glucose in whole blood samples. Greiner tubes inhibit glycolysis instantly and we previously demonstrated that their stability upholds for up to 96 hours at room temperature. Nevertheless, our study also revealed, that Greiner tubes require special pre-analytical caution to ensure a full dissolution of the glucose stabilizing granula. The recommended inversion of ten times after blood collection proved to be insufficient and displays a risk in every day blood collection routine. Furthermore, this tube is an extra tube, just for one analyte. Regarding blood patient management it would be preferable to measure glucose concentrations out of regularly used blood collection tubes such as Lithium Heparin (LH) tubes. BD recently introduced Barricor, a special LH tube, which can be centrifuged right after blood collection at $4000 \mathrm{x} \mathrm{g}$ for three minutes and might serve as an alternative to the Greiner tubes. We aimed to examine whether rapid centrifugation and therefore rapid plasma and blood cell separation could replace the extra glucose tube and whether this would lead to a long-term stable glucose concentration in Barricor tubes.

Methods: Whole blood sample of 14 volunteers were collected in two Greiner FC mixture tubes and in two BD Barricor tubes following the manufacturer's recommendations. One set of Barricor and Greiner tubes was instantly centrifuged and the Greiner supernatant was transferred into a new tube. The second set of Barricor and Greiner tubes was centrifuged one hour after blood collection, mimicking a delayed glucose measurement after a prolonged sample transportation time. For both tube sets, initial glucose measurements were performed by using the Glucose Hexokinase method on the Dimension Vista ${ }^{\circledR} 1500$ System (Siemens Healthineers, Eschborn, Germany). After the initial glucose measurement, samples were stored at $4^{\circ} \mathrm{C}$ and re-measured on a daily basis. The last glucose value was obtained at 96 hours. Differences in the initial glucoses concentrations between the different tubes as well as long-term stability were statistically evaluated using Friedman ANOVA and Wilcoxon signed-rank-sum tests.

Results \& Conclusion: Both Greiner tubes displayed similar initial glucose concentrations that were significantly higher than the glucose concentration in the delayed processed Barricor tube. The latter showed the lowest initial glucose concentration of all four tested tubes. Greiner tubes confirmed their good long-term stability of up to 96 hours with no significant glucose decrease. On the other hand, glucose concentrations decreased significantly with each measurement in both tested Barricor settings, which could be explained by continued glycolysis by the remaining platelet in the plasma. Not even the instant and rapid centrifugation led to an acceptable glycolysis inhibition, proving that there is momentarily no alternative to an extra glucose blood collection tube. 


\title{
DGKL-P111
}

\section{Blutgruppen gestützte Aufdeckung von Patientenverwechslung bei der Blutprobenentnahme für klinisch chemische Laboruntersuchungen}

Severin Hahn

Hamburg, Germany

Therapie eine erhebliche Rolle. Aufgrund der hohen Frequenz mit welcher dieses geschieht und den damit einhergehenden Komplikationen hat sich in der angloamerikanischen Fachliteratur ein eigenes Fachwort dafür etabliert. Unter dem Begriff „Wrong Blood in Tube“ (Kurz „WBIT“) werden alle Verwechslungen Zusammengefasst, welche dazu führen, dass eine Blutprobe nicht vom Labor entsorgt oder zurückgeschickt wird, sondern einem Falschen Patienten zugeordnet wird. WBIT stellt ein denkbar großes Problem in der Labormedizin, und damit in der Diagnostik, dar, da es zu Fehldiagnosen und Fehltherapien, im schlimmsten Fall mit physischen, psychischen oder sogar letalen Schäden, kommen kann. Da sich in der internationalen Fachliteratur zum Teil widersprüchliche bzw. nicht auf den Norddeutschen Raum beziehbare Daten zur Häufigkeit der WBIT finden ist es das Ziel dieser Diplomarbeit konkrete und verwertbare Daten zu diesem Thema im Norddeutschen Raum zu präsentieren. 1.2.2 Ursachen

Die Ursachen für WBIT sind vielfältig und gut erforscht. Es handelt sich um 3 mögliche Abschnitte im Prozess der Blutanalyse die Fehlerquellen bieten welche zu Blutprobenverwechslungen führen können:

Präanalytisch - Von der Auftragsstellung zur Blutentnahme über die Ankunft der Blutprobe im Labor bis hin zu allen Vorbereitungen die für die tatsächliche Analyse benötigt werden

Analytisch - Laboratorische Analyse der Blutproben

Postanalytisch - Von der Übermittlung der Befunde über deren Interpretation und ihrer ärztlichen Handhabung

1. Preanalytical Errors:

These errors are defined as the errors occurring from physician order to analytic phase, including test request, patient identification, collection, transportation, and preparation for analysis. For better classification and monitoring of preanalytical phase, it is subdivided to errors that occur outside the laboratory and errors that occur under the control of laboratory as Da Rin has mentioned in his study.[11] Outside Laboratory: inappropriate test request, order entry errors, misidentification of patient, inappropriate container, improperly labeled container, inadequate sample collection and transportation, specimen collected from infusion route, inadequate sample/anticoagulant volume ratio, and insufficient sample volume. Within laboratory: sorting and routing errors, pour-off errors, and labeling errors.

2. Analytical Errors:

These errors occur during the test, and include equipment malfunction, sample mix-ups, interference, undetected failure in quality control, and procedure not followed.

3. Postanalytical Errors:

These errors happen after the test is conducted, and include failure in reporting, erroneous validation of analytical data, improper data entry, and excessive turnaround time.

https://www.ncbi.nlm.nih.gov/pmc/articles/PMC4049056/

However, the preanalytical phase can be further subdivided.

\section{Posterwalk DVTA}

\section{DVTA-P01}

\section{Dried Blood Spots - Extraktion und Analyse von stoffwechselrelevanten Aminosäuren}

\author{
Birgit Krause; Otmar Löhnertz \\ Hochschule Geisenheim University, Zentrum für Biowissenschaften, Geisenheim, Germany
}

Due to participating in the 2017 and 2018 special assays in dried blood spots scheme of the European Research Network for Diagnosis of Inherited Metabolism (ERNDIM) it was intended to work out dried blood spot (DBS)-extractions and extract analysis for clinically-relevant amino acids in a worldwide method comparison for an onwards certification process. Analytics from DBS, which are already used for new inborn screening nationwide in Germany since 2005, turns out increasingly to be focussed [1-4].

DBS were extracted in $1500 \mu \mathrm{l}$ tubes while adding following chemicals in a ninety minutes operation: Trichloroacetic acid, Tris(2-carboxyethyl) phosphine hydrochloride solution, 5-sulfosalicylic acid dihydrate. By shaking of these tubes and by cooled storage the amino acids went into solution and protein was precipitated. Following this, extracts were centrifugated and diluted for analysis with sample ditution buffer ( $\mathrm{pH} 2,2$ ) on an amino acid analyzer [5]. A special short application was set up for determination of the relevant amino acids.

The worked out extraction method with following analysis on an amino acid analyzer was placed within one- to twofold standard deviation in this worldwide method comparison involving 75 participiants on this pilot project. The gathered amino acids have been allo-isoleucine, homo-cysteine, isoleucine, leucine, phenylalanine, tyrosine and valine. 
A stable result of amino acid homo-cysteine was obtained by reducing disulfidbonds with tris-carboxyethylphosphine. Only one third of the participants took part in the analysis of this physiological amino acid. So it can be supposed that this determination is not well known. DBS are a good alternative for multiple analysis concerning storage and transport, as well as long stability. Extract analysis of amino acids are well reproducible and quickly and reasonable determined with the wide spread amino acid analyzers.

\section{Literature:}

[1] Lindner, C et al. : Comparison between dried blood spot and plasma sampling for therapeutic drug monitoring of antiepileptic drugs in children with epilepsy: A step towards home sampling. Clinical Biochemistry (2017); 50: 418-424.

[2] Wenkui, L et al. : LC-MS/MS bioanalysis of loratadine (Claritin) in dried blood spot (DBS) samples collected by subjects in a clinical research study. Journal of Chromatography B (2015); 983-984: 117-124.

[3] Smit, PW et al. : Systematic review of the use of dried blood spots for monitoring HIV viral load and for early infant diagnosis. Plos ONE (2014); 9(3): e86461.

[4] Fingerhut R, Röschinger, W and Heck, M: A Rapid and Sensitive UPLC-MS/MS-Method for the Separation and Quantification of BranchedChain Amino Acids from Dried Blood Samples of Patients with Maple Syrup Urine Disease (MSUD). Int. J. Neonatal Screen. (2016); 2(2).

[5] Europäisches Arzneibuch: Aminosäureanalyse im Europäischen Arzneibuch (2014); 8.0/2.02.56.00: 124-134.

\section{DVTA-P02}

\section{Crossmatching as an obligate, indication-independent or indication-dependent compatibility control in the medicine of transfusion - an international comparison}

Sonja Reischenböck; Marco Kachler

Fachhochschule Kärnten, Biomedizinische Analytik, Klagenfurt, Austria

\section{Background:}

Crossmatching is an obligatory testing method, which is conducted in Austria before every transfusion. It is an additional safety precaution for the AB0-testing and the antibody screening. The purpose of this test is to ensure the compatibility between the blood donor and the recipient as well as to minimize the risk of a transfusion incident.

As opposed to this, in Switzerland the so called “Type \& Screen”- System is being used. It provides an implementation of crossmatching only if there is an indication, such as a positive result in the Antibody screening.

\section{Material and Methods:}

The present study will try to answer the question if the different approaches have additional benefits for the recipient.

Additionally the different procedures will be compared with the systems of Germany and the United States of America. Therefor, the countryspecific regulations in the laboratory as well as the haemovigilance reports since 2012 will be used.

Furthermore, relevant publications will be included in the course of discussion.

Results:

According to the heamovigilance reports, it was not possible to find any coherence between the different procedures used and the frequency of relevant transfusion incidents. One reason for this is the variant willingness of reporting those incidents, which makes a comparison problematic. Another important aspect for assessing the procedure would be the estimation of the expenditure, but the data at hand was not detailed enough for such an assessment.

Additionally, it should be considered, if due to the increase of the ethnic variance in the population, the frequence of rare antibodies will rise as well, which can most likely be detected due to the procedure of crossmatching.

\section{DVTA-P03}

\section{Assessment of evidence for the determination of procalcitonin in routine diagnostics based on the AWMF guidelines}

Elisa Wieser; Marco Kachler

Fachhochschule Kärnten, Biomedizinische Analytik, Klagenfurt, Austria

\section{Background:}

Procalcitonin (PCT) is a parameter that only has a minor role in Austria. In the literature, Procalcitonin is frequently mentioned in connection with C-reactive protein (CRP) and defined as an inflammatory parameter. PCT is used in sepsis diagnostics, antibiotic control of bacterial infections, and as prognosis marker for infections. However, the experts are divided in this regard. Consequently, the question arises on which evidence-based background the determination of PCT is useful? To determine the status of PCT in terms of diagnosis, treatment and other medical questions, a comparison of guidelines seemed as useful tool.

Material and Methods:

This study assesses the evidence for the routine determination of PCT based on the guidelines of the Association of the Scientific Medical Societies in Germany (AWMF). The search for guidelines was conducted in the online database of the AWMF with "Procalcitonin" as search 
term. Only valid guidelines of level S3 were used. The evidence and consensus on PCT were evaluated based on six relevant guidelines including pneumonia, cough, Crohn's disease, urinary tract infections and hospital antibiotic use.

Results:

The results show that only on occasion evidence was explicitly given for PCT. In most cases, PCT was mentioned in connection with CRP, as well as general diagnostics. That made it difficult to find solid statements about the functions and usefulness of PCT. In terms of controlling the antibiotic therapy duration in the context of pneumonia as well as in the general antibiotic care in hospitals, PCT is a very useful biomarker. Another guideline discusses the cost-value ratio of PCT determination.

Since the guideline search is limited to only one database, only a few statements can be derived directly for PCT. The analysis of the primary literature would be more informative at this point in order to evaluate the quality of the evidence.

\title{
DVTA-P04
}

\section{Norovirus und Rotavirus im Krankenhaus - RT-PCR vs. ELISA - Kosten vs. Sensitivtät}

Susann Neumann; Dirk Bandt

Oberlausitz-Kliniken gGmbH, Institut für Labordiagnostik, Mikrobiologie und Krankenhaushygiene, Bautzen, Germany

Evaluierung der RT-PCR für Norovirus und Rotavirus mittels BD MAX ${ }^{\mathrm{TM}}$ Enteric Viral Panel-NR unter analytischen, diagnostischen und ökonomischen Aspekten für die Oberlausitz-Kliniken gGmbH

\section{Einleitung:}

Norovirus und Rotavirus sind weltweit Hauptverursacher der akuten viralen Gastroenteritis. Eine Früherkennung ist entscheidend, um eine nosokomiale Verbreitung im Krankenhaus zu verhindern. Die RT-PCR stellt den Goldstandard in der Diagnostik des Norovirus und Rotavirus dar.

\section{Ziel der Arbeit:}

Wie ist die diagnostische Genauigkeit der RT-PCR im Vergleich zum ELISA für Norovirus und Rotavirus? Wie kann die Arbeitsorganisation für die Analytik der Stuhlviren in der Oberlausitz-Kliniken gGmbH unter Berücksichtigung von ökonomischen Aspekten gestaltet werden?

\section{Material und Methoden:}

Ein Methodenvergleich mit 140 Stuhlproben aus der Oberlausitz-Kliniken gGmbH wurde für die 2er-Multiplex-RT-PCR „BD MAX ${ }^{\mathrm{TM}}$ Enteric Viral Panel-NR“ und den ELISAs „RIDASCREEN® Norovirus der 3rd Generation“ und „RIDASCREEN® Rotavirus“ durchgeführt. Sensitivität und Spezifität wurden im Vergleich zur Diagnose ausgewertet. Ökonomische Aspekte wurden betrachtet und bewertet. Die Möglichkeiten der Arbeitsorganisation für Stuhlviren wurden im Hinblick auf Reagenzkosten, Arbeitsaufwand und Flexibilität der Bearbeitung und Befundlaufzeiten bewertet.

\section{Ergebnisse:}

Die Sensitivität für die RT-PCRs wurde für Norovirus mit 93,9 \% und für Rotavirus mit 71,4 \% ermittelt, im Vergleich zum ELISA mit 57,5 \% und 57,1 \%. Die Spezifität liegt beim molekularbiologischen Test bei 99,0 \% bzw. 100 \% zu 96,0 \% bei den ELISA-Testen. Die Reagenzmehrkosten der Multiplex-PCR für Noroviren und Rotaviren betragen mehr als 23.100,00 € für das Jahr 2017. Mit Einführung der 5er-Multiplex-PCR „BD MAX ${ }^{\text {TM}}$ Enteric Viral Panel“ kann unter Berücksichtigung der Meldepflichten in Sachsen die flexibelste und schnellste Abarbeitung der Stuhlviren ohne Altersbeschränkung erzielt werden. Eine Kompensation der steigenden Reagenzkosten durch höhere Erlöse aus den Fallpauschalen des G-DRG-Systems ist nur im geringem Umfang möglich. Eine höhere Bewertung findet nur bei Kleinkindern und PatientInnen mit schweren Komorbiditäten statt.

\section{Zusammenfassung:}

Es konnte gezeigt werden, dass die RT-PCR den Goldstandard zur Diagnostik der Noroviren und Rotaviren darstellt. Eine Umstellung auf molekularbiologische Nachweisverfahren ist durch steigende Kosten im Labor gekennzeichnet, bietet aber die Möglichkeit, durch Automatisierung die Nachweise zeitnah zur Verfügung zu stellen und eine Versorgung der PatientInnen auf derzeit besten medizinischen Erkenntnissen zu bieten. Eine nosokomiale Ausbreitung kann am gezieltesten mit bekannten Virus verhindert werden, dadurch können teure und invasive Untersuchungen im stationären Bereich minimiert und Stationsschließungen und Personalausfall verringert werden.

\section{DVTA-P05}

\section{Einfluss einer Präanalytik-Schulung auf Laboruntersuchungen}

\author{
Cornelia Mäder-Porombka
}

Ruprecht-Karls-Universität Heidelberg, Allgemeinmedizin und Versorgungsforschung, Heidelberg, Germany

Hintergrund: Fehler in der Labormedizin sind vorwiegend auf präanalytische Ursachen zurückzuführen und ereignen sich meist schon vor Eintreffen der Probe im Labor. Fehlerhafte Blutentnahme (BE), wie beispielsweise die ungenügende Füllung von Röhrchen, führen zu 
falschen Messergebnissen und zusätzlichen Analysekosten, die Dauer der Analyse erhöht sich und die Gesundheit von Patienten und Angestellten wird gefährdet. Bisher werden Mitarbeiter außerhalb des Labors kaum hinsichtlich der Fehlervermeidung im Bereich der Präanalytik geschult. Fragestellung: Kann durch eine maßgeschneiderte Präanalytik-Schulung die Anzahl präanalytisch fehlerhafter Proben signifikant verringert werden? Methodik: Auf einer chirurgischen Station des Universitätsklinikums Heidelberg wird durch teilnehmende Beobachtungen (TB) die BE vor (prä, $n=6$ ) und nach (post, $n=6$ ) der Schulung mit den klinikinternen Richtlinien verglichen. Basierend auf den Ergebnissen der prä-TB wird eine 30-minütige Schulung konzipiert und durchgeführt. Zusätzlich soll durch unterstützende Infomaterialien die Intervention Fidelity erhöht werden. Hauptzielkriterium ist die Anzahl der durch das Labor kommentierten Gerinnungsuntersuchungen; Nebenzielkriterien sind die Anzahl hämolytischer Untersuchungsmaterialien und die Qualität der Durchführung der BE entsprechend der klinikinternen Richtlinien. Bei Signifikanzniveau $\alpha=0,05$ und klinisch relevanten Effekt $\delta=1,6 \%$ liegt die berechnete Power bei $75,5 \%$. Ab einer Reduktion kommentierter Gerinnungsuntersuchungen von 6,6\% aller Aufträge auf 5,0\% kann von einem klinisch relevanten Effekt gesprochen werden, da ab dann die potentiellen Kosten für eine Schulung durch eingesparte Kosten gedeckt würden. Zur statistischen Auswertung des Unterschieds vor und nach der Schulung wird der Chi-Quadrat-Test verwendet. Ergebnisse: Die chirurgische Station hatte 201711.873 Gerinnungsuntersuchungen in Auftrag gegeben. 6,6\% davon wurden im Labor mit Kommentaren versehen wegen 10- bzw. 20-prozentiger Unter- und Überfüllung. Die Häufigkeiten kommentierter Gerinnungsuntersuchungen werden von Juni bis August 2018 erneut erfasst und mit den Häufigkeiten von Juni bis August 2017 (zur derselben Jahreszeit vor Bekanntwerden der Studie), Januar bis März 2018 (Bekanntwerden der Studie und TB) und Oktober bis Dezember 2017 (direkt vor Bekanntwerden der Studie) verglichen. Zudem wird ein Konfidenzintervall von 95\% berechnet. Schlussfolgerung: Es wird erwartet, dass durch die maßgeschneiderte Schulung positive Effekte auf die Fehlervermeidung in der Präanalytik erzielt werden. Weiterhin geklärt werden müsste, wie häufig entsprechende Schulungen wiederholt werden sollten und inwieweit langfristig eine positive Kostenbilanz erzielt werden kann.

\title{
DVTA-P06
}

\section{Does the mixing ratio between blood and citrate (tube fill volume) influence the results of the two global tests prothrombin time (PT) and partial thromboplastin time (aPTT)?}

\author{
Christina Eggerer; Marco Kachler \\ Fachhochschule Kärnten, Biomedizinische Analytik, Klagenfurt, Austria
}

Introduction: In routine coagulation testing, laboratories are often presented with under-filled sodium citrate tubes, which are suspected to produce incorrect coagulation values. Consequently, most laboratories request a second venipuncture, when confronted with a collection tube filled under $90 \%$ to ensure a correct mixing ratio between citrate and blood (1:10).

Methods: The aim of the present study was to evaluate the impact of different sample volumes on coagulation testing. This is demonstrated by comparing results of two global tests: PT and aPTT, which represent the most common coagulation analyses.

The hypothesis was tested using existing studies selected by a literature search on "PubMed".

Results and Discussion: After summarizing all results, the following conclusion can be drawn: it is very likely, that also a tube fill volume less than $90 \%$ can be allowed in coagulation testing. Significant differences in analysis results occur nearly always at clearly lower tube filling volumes, which range between $50 \%$ and $90 \%$ for PT and $50 \%$ and $100 \%$ for aPTT, depending on the study and the citrate concentration of the tubes.

Nevertheless further studies with larger study populations are needed to verify this conclusion, because this would help to minimize patient suffering and keep costs at a lower level.

\section{DVTA-P07}

\section{Hypogalactosylation of immunoglobulin $\mathrm{G}$ in rheumatoid arthritis: relationship to HLADRB1shared epitope, anticitrullinated protein antibodies, rheumatoid factor, and correlation with inflammatory activity}

Christian Schwedler'; Thomas Häupl ${ }^{1}$; Ulrich Kalus²; Veronique Blanchard 3 ; Gerd-Rüdiger Burmester'; Denis Poddubnyy ${ }^{4}$; Berthold Hoppe ${ }^{5}$ ${ }^{1}$ Charité-Universitätsmedizin Berlin, Department of Rheumatology, Berlin,Germany; ${ }^{2}$ Charité-Universitätsmedizin Berlin, Institute of Transfusion Medicine, Berlin, Germany; ${ }^{3}$ Charité-Universitätsmedizin Berlin, Institut für Laboratoriumsmedizin, Klinische Chemie und Pathobiochemie, Berlin, Germany; ${ }^{4}$ Charité-Universitätsmedizin Berlin, 5Department of Gastroenterology, Infectiology and Rheumatology, Berlin, Germany; ${ }^{5}$ Charité-Universitätsmedizin Berlin, Institute of Laboratory Medicine, Berlin, Germany

Background: Galactosylation of immunoglobulin G (IgG) is reduced in rheumatoid arthritis (RA) and assumed to correlate with inflammation and altered humoral immunity. IgG hypogalactosylation also increases with age. To investigate dependencies in more detail, we compared IgG hypogalactosylation between patients with RA, patients with axial spondyloarthritis (axSpA), and healthy control subjects (HC), and we 
studied it in RA on the background of HLA-DRB1 shared epitope (SE), anticitrullinated protein antibodies (ACPA), and/or rheumatoid factor (RF) status.

Methods: Patients with RA ( $\mathrm{n}=178)$, patients with axSpA $(\mathrm{n}=126)$, and HC $(\mathrm{n}=119)$ were characterized clinically, and serum IgG galactosylation was determined by capillary electrophoresis. Markers of disease activity, genetic susceptibility, and serologic response included C-reactive protein (CRP), erythrocyte sedimentation rate, DAS28, SE, HLA-B27, ACPA, and RF. Expression of glycosyltransferases was estimated from transcriptome data for B-cell development and differentiation to plasma cells.

Results: IgG hypogalactosylation was restricted to RA and associated with increasing CRP levels. In axSpA, IgG hypogalactosylation was comparable to HC and only marginally increased upon elevated CRP. Restriction to RA was maintained after correction for CRP and age. Treatment with sulfasalazine resulted in significantly reduced IgG hypogalactosylation even after adjusting for age, sex, and CRP. Gene expression data indicated that the key enzyme for galactosylation of immunoglobulins, B4GALT3, is expressed at lower levels in B cells than in plasma cells. Conclusion: Increased IgG hypogalactosylation in RA but not in axSpA points to humoral immune response as a precondition. Reduced B4GALT3 expression in B cells compared with plasma cells supports relatedness to early B-cell triggering. The differential influence of RA treatment on IgG hypogalactosylation renders it a potential diagnostic target for further studies.

\section{DVTA-P08}

\section{Neisseria gonorrhoeae - comparison of phenotypic and genotypic methods for antimicrobial susceptibility testing}

Katharina Walter; Marco Kachler

Fachhochschule Kärnten, Biomedizinische Analytik, Klagenfurt, Austria

\section{Background:}

The World Health Organization (WHO) estimates that Neisseria gonorrhoeae is the third leading cause of sexually transmitted diseases (STDs) worldwide. In 2014 the incidence in Europe was about 20 cases per 100.000 inhabitants. The major problem is the increase in resistance of N. gonorrhoeae strains. In 2017 a total of 274 gonococcal isolates were analyzed by the National Reference Centre in Austria (AGES). Of the tested strains all isolates were susceptible to ceftriaxone, $4 \%$ showed resistance to cefixime, 50\% to ciprofloxacin, $4 \%$ to azithromycin, $43 \%$ to tetracycline and $17 \%$ showed resistance against penicilline.

Conventional methods for antimicrobial susceptibility testing of N. gonorrhoeae are phenotypic methods like agar dilution or E-test. However these methods are time consuming and sensitive to several influences. In contrast genotypic methods, could establish these resistances by detection of mutations in some regions of the responsible gene(s), saving time and money. Additionally PCR based methods, are already used for the detection of N. gonorrhoeae, because of their higher sensitivity compared to culture methods.

Material and Methods:

The aim of the present study is to relate the quality of genotypic results for the detection of antibiotic resistance in clinically relevant strains of N. gonorrhoeae to those of phenotypic methods.

To answer this question a systematic literature research was conducted and 10 publications about this issue where chosen as basis for this review study.

Results:

Within these publications antimicrobial susceptibility testing of 7 different antibiotics was implemented by phenotypic and genotypic methods. Therefor it was possible to determine the sensitivity and specificity of the genotypic methods. Sensitivity spread from $50 \%$ to $100 \%$ and specificity from $27.7 \%$ to $100 \%$. The 10 publications showed that, if all genotypic combinations are detected and an applicative definition for resistance is used, the genotypic methods are able to accomplish similar results when compared to the phenotypic methods.

\section{DVTA-P09}

\section{Quantitative analysis of KIT D816V in patients with mastocytosis - suitability of ctDNA compared to gDNA}

Angelika Duda ; Mohamad Jawhar', Michael Neumaier'; Andreas Reiter ${ }^{2}$, Verena Haselmann ${ }^{1}$;

${ }^{1}$ Medizinische Fakultät Mannheim, Universität Heidelberg, Institut für Klinische Chemie, Mannheim, Germany; ${ }^{2}$ Universitätsmedizin Mannheim, Hämatologie und Onkologie, Mannheim, Germany

BACKGROUND: Mastocytosis is characterized by abnormal growth and accumulation of mast cells in skin (cutaneous mastocytosis) and/or various organs (systemic mastocytosis, SM). SM can present in four different major forms including indolent SM (ISM), SM with an associated hematologic neoplasm (SM-AHM), aggressive SM (ASM) or mast-cell leukemia (MCL). Up to $90 \%$ of patients with SM harbor a somatic gain-offunction KIT D816V mutations that is crucial for disease evolution, diagnosis and targeted treatment with tyrosine-kinase-inhibitor (e.g. midostaurin, a multi-tyrosine kinase/KIT-inhibitor). It has been shown that KIT D816V level is associated with disease activity, subtype and survival and can be used to monitor therapy response and disease progression.. As correlation of levels depends on subtype, the questions arise whether circulating tumor DNA (ctDNA) released into the circulating or genomic DNA (gDNA) from leukocytes is more adequate for mutation detection. 
METHODS: As digital droplet PCR (ddPCR) allows an absolute quantification with a detection limit of mutant alleles in a background of wildtype alleles up to $0.01 \%$, we decided to establish a KIT D816V ddPCR assay on Biorad X200 system in our laboratory. The assay was carried out according to manufacturer's instructions with minor modifications. For analysis of gDNA from whole-EDTA-blood, $6 \mu \mathrm{lgDNA}$ isolated from $200 \mu \mathrm{l}$ blood eluted in $200 \mu \mathrm{l}$ was used; for analysis of ctDNA from plasma, $6 \mu \mathrm{l}$ per triplicate of cfDNA isolated from 3 ml EDTA-blood eluted in $70 \mu l$ was used.

RESULTS: Establishment of our assay identified a limit of detection of $0.04 \%$ with at least five positive droplets to be detected for calling a sample positive. For validation, we participated in a round-robin trial demonstrating that our assay results perfectly matches the results of the reference laboratory with a very low coefficient of variation (VKmean $=0.05$ ). Consequently, we decided to analyze blood and plasma samples from so far 16 patients with different types of SM with our assay. Up to now, results of mutational analysis from gDNA and ctDNA show an overall agreement of $100 \%$. Moreover, in most cases the mutant allele fraction (MAF) is comparable, but in some cases differentiates substantially with lower levels found in ctDNA.

CONCLUSION: Our first results demonstrate a high level of correlation for plasma- and blood-based mutational testing of KIT D816V of patients suffering from SM indicating that ddPCR might be suitable for follow-up of patients as an alternative to qPCR. However, further testing will be necessary to evaluate whether correlation with disease activity of ctDNA might be superior to analysis of gDNA depending on SM subtype.

\section{DVTA-P10}

\section{Herstellung von Kontrollproben für seltene genetische Variationen mittels Overlap-Extension-PCR}

Romy Eichner; Laura Mirbach; Jasmin Rieser; Florentine Stix; Michael Neumaier; Verena Haselmann

Universitätsmedizin Mannheim, Institut für Klinische Chemie, Mannheim, Germany

Einleitung: Seit Entwicklung der Hochdurchsatzsequenzierung hat sich unser Verständnis der Genetik zahlreicher Erkrankungen, darunter auch komplexer polygenetischer Erkrankungen grundlegend verändert. Somit spielen heute immer mehr genetische Veränderungen zur Risiko- oder Prognoseabschätzung sowie zur differentialdiagnostischen Abklärung diverser Erkrankungen, aber auch bei der Auswahl und Dosierung unterschiedlicher Pharmaka im Sinne einer pharmakogenetischen Analyse eine wichtige Rolle. Für molekularbiologische Untersuchungen bedeutet dies ein immer breiter werdendes Spektrum, das einen bei zunehmender Anzahl zu untersuchender, zum Teil seltener Sequenzvariationen (v.a. bei der steigenden ethnischen Diversität) vor die Herausforderung nach geeigneten Kontrollproben stellt. Da dies leider oftmals nicht oder nur nach Jahren gelingt, gewinnt die Herstellung von eigenem Kontrollmaterial an Bedeutung. Hierfür kommt in erster Linie das Design entsprechender Zelllinien mittels Klonierung in Frage sowie als schnellere, kostengünstigere Alternative bei nur geringem Probenaufkommen die Herstellung mutierter Amplikons mittels Overlap-Extension-PCR (OE-PCR).

Material und Methoden: Für die Herstellung mutierter Amplikons mittels zielgerichteter Mutagenese durch OE-PCR müssen zunächst zwei Amplikons hergestellt werden, die eine Überlappung von ungefähr 50bp aufweisen, wobei die überlappenden Primer die zu induzierende Sequenzvariation enthalten. Nach äquimolarem Poolen der beiden PCR-Produkte erfolgt eine zweite PCR mit Hilfe der äußeren oder alternativ weiter innenliegenden nested-Primern.

Ergebnisse und Diskussion: Der Einsatz der OE-PCR zur Herstellung von Kontrollproben soll am Beispiel von zwei Proben für EGFR T790M und PRF1 W374X gezeigt werden. Hier wird durch typische Pitfalls deutlich, dass die Effizienz sowie Spezifität der OE-PCR wesentlich von der Spezifität aller verwendeten Primersequenzen abhängig ist. Möglichkeiten der Optimierung zeigen die Überlegenheit der Verwendung von nested-Primern, der damit verbundenen Reduktion der Anzahl an Aufreinigungen sowie des alternativen Einsatzes von Verdünnungen der PCR-Produkte oder Veränderungen des Amplifikationsprotokolls.

Zusammenfassung: Insgesamt zeigen die Ergebnisse, dass mittels OE-PCR einfach und schnell Kontrollproben für seltene Sequenzvariationen hergestellt werden können. Bei dem Design ist vor allem auf die Spezifität der einzelnen eingesetzten Primerpaare sowie der Verwendung von nested-Primern für die zweite Amplifikation zu achten, die wiederholte Aufreinigungsschritte ersetzen kann.

\section{DVTA-P11}

\section{Analyse des BRAF V600E Mutationsstatus im Plasma - Ergebnisse eines Plattformvergleichs digitaler Verfahren}

Laura Mirbach; Angelika Duda; Ingrid Brechtel; Romy Eichner; Michael Neumaier; Verena Haselmann

Universitätsmedizin Mannheim, Institut für Klinische Chemie, Mannheim, Germany

Einleitung: Seit der Entwicklung zielgerichteter Therapeutika hat die Bedeutung des genetischen Tumorprofilings stetig zugenommen. Voraussetzung für die Administration einer BRAF Inhibitor-Therapie (BRAFi) bei Patienten mit metastasiertem Malignem Melanom ist der molekulargenetische Nachweis einer BRAF V600 Mutation, welcher derzeit aus Gewebeproben bestimmt wird. Durch die Analyse zirkulierender Tumor-DNA kann diese Analyse auch direkt aus dem Blut erhoben werden mit den Vorteilen einer minimal-invasiven Diagnostik in Echtzeit, die der Tumorheterogenität und Tumorevolution gerecht wird und ein therapeutisches Monitoring ermöglicht. Für die Einführung in die 
Routine ist allerdings eine Standardisierung der hochsensitiven Nachweisverfahren essentiell, so dass sich die Frage nach geeigneten Untersuchungsmethoden stellt.

Material und Methoden: 46 Patienten mit metastasiertem Melanom vor Therapiebeginn oder zum Zeitpunkt einer nachweisbaren Progression unter BRAFi Therapie wurden in den Methodenvergleich eingeschlossen. Der Mutationsstatus aus FFPE-Gewebeproben wurde mittels Sanger Sequenzierung von Pathologischen Instituten im Rahmen der Routinediagnostik bestimmt, $123 \mu$ l zellfreie DNA (cfDNA) wurden mittels OncoBEAM V600E Assay (Sysmex Inostics) und 7 $\mu$ cfDNA mittels ddPCR BRAF V600 Screening Kit (Bio-Rad) entsprechend der Herstellerangaben untersucht.

Ergebnisse: Insgesamt zeigte sich für die Konkordanz des Tumormutationsstatus zwischen Gewebe und Plasma eine Übereinstimmung von 93,5 \% für den OncoBEAM BRAF V600E Assay und von 78,3 \% für das ddPCR BRAF V600 Screening Kit. Entsprechend des von der ermittelten Kopienzahl abhängigen berechneten Limit of Detection (LOD) des ddPCR Assays sowie der mittels BEAMing bestimmten Mutant Allele Fraction (MAF), lag in 6/8 falsch negativen Testergebnissen die MAF unterhalb des LOD.

Diskussion: Die Ergebnisse zeigen eine insgesamt hohe Konkordanz zwischen Gewebe- und Plasma-basierter Diagnostik. Hervorzuheben ist die Abhängigkeit des LOD vom Input, so dass bei der ddPCR entsprechend eine Untersuchung in Replikaten zwingend erforderlich sein sollte. Berücksichtigt man die LOD ergibt sich eine 100\% Konkordanz zwischen den digitalen Untersuchungsverfahren, insbesondere auch hinsichtlich der zum Gewebe diskordanten Ergebnisse.

Zusammenfassung: Zusammenfassend zeigen die Ergebnisse, dass beide digitale Verfahren plausible Ergebnisse liefern. Unter der Voraussetzung der Berücksichtigung präanalytischer Faktoren sind beide Plattformen für eine standardisierte routinetaugliche Untersuchung zirkulierender Tumor-DNA geeignet und als Alternative zur Gewebe-basierten Diagnostik einsetzbar. 